\title{
DIRECT OXYGEN ABUNDANCES FOR LOW-LUMINOSITY LVL GALAXIES*
}

\author{
Danielle A. Berg ${ }^{1}$, Evan D. Skillman ${ }^{1}$, Andrew R. Marble ${ }^{2,3}$, Liese van Zee ${ }^{4}$, Charles W. Engelbracht ${ }^{2}$, \\ Janice C. Lee ${ }^{5}$, Robert C. Kennicutt JR. ${ }^{6,7}$, Daniela Calzetti ${ }^{8}$, Daniel A. Dale ${ }^{9}$, and Benjamin D. Johnson ${ }^{10}$ \\ ${ }^{1}$ Institute for Astrophysics, University of Minnesota, 116 Church St. SE, Minneapolis, MN 55455, USA; berg@ astro.umn.edu, skillman@astro.umn.edu \\ ${ }^{2}$ Steward Observatory, University of Arizona, 933 N Cherry Ave., Tucson, AZ 85721, USA; cengelbracht@as.arizona.edu \\ ${ }^{3}$ National Solar Observatory, 950 N Cherry Ave., Tucson, AZ 85719, USA; amarble@nso.edu \\ ${ }^{4}$ Astronomy Department, Indiana University, 727 East 3rd Street, Bloomington, IN 47405, USA; vanzee@ astro.indiana.edu \\ ${ }^{5}$ STScI, 3700 San Martin Drive, Baltimore, MD 21218, USA; jlee@ stsci.edu \\ ${ }^{6}$ Institute of Astronomy, University of Cambridge, Madingley Road, Cambridge CB3 OHA, UK \\ ${ }^{7}$ Steward Observatory, University of Arizona, Tucson, AZ 85721, USA \\ ${ }^{8}$ Department of Astronomy, University of Massachusetts, 710 North Pleasant Street, Amherst, MA 01003, USA; calzetti@astro.umass.edu \\ ${ }^{9}$ Department of Physics and Astronomy, University of Wyoming, 1000 E. University, Laramie, WY 82071, USA; ddale@ uwyo.edu \\ ${ }^{10}$ Institut d'Astrophysique de Paris, UMR 7095, 98 bis Bvd Arago, 75014 Paris, France; johnson@iap.fr \\ Received 2012 January 9; accepted 2012 May 24; published 2012 July 12
}

\begin{abstract}
We present MMT spectroscopic observations of H II regions in 42 low luminosity galaxies in the Spitzer Local Volume Legacy survey. For 31 of the 42 galaxies in our sample, we were able to measure the temperature sensitive [O III] $\lambda 4363$ line at a strength of $4 \sigma$ or greater, and thus determine oxygen abundances using the "direct" method. Our results provide the first "direct" estimates of oxygen abundance for 19 of these galaxies. "Direct" oxygen abundances were compared to $B$-band luminosities, $4.5 \mu \mathrm{m}$ luminosities, and stellar masses in order to characterize the luminosity-metallicity and mass-metallicity relationships at low luminosity. We present and analyze a "Combined Select" sample composed of 38 objects (drawn from a sub-set of our parent sample and the literature) with "direct" oxygen abundances and reliable distance determinations (based on the tip of the red giant branch or Cepheid variables). Consistent with previous studies, the $B$ band and $4.5 \mu \mathrm{m}$ luminosity-metallicity relationships for the 38 objects were found to be $12+\log (\mathrm{O} / \mathrm{H})=(6.27 \pm 0.21)+(-0.11 \pm 0.01) M_{B}$ and $12+\log (\mathrm{O} / \mathrm{H})=(6.10 \pm 0.21)+(-0.10 \pm 0.01) M_{[4.5]}$ with dispersions of $\sigma=0.15$ and 0.14 , respectively. The slopes of the optical and near-IR $L-Z$ relationships have been reported to be different for galaxies with luminosities greater than that of the LMC. However, the similarity of the slopes of the optical and near-IR $L-Z$ relationships for our sample probably reflects little influence by dust extinction in the low luminosity galaxies. For this sample, we derive a mass-metallicity relationship of $12+\log (\mathrm{O} / \mathrm{H})=(5.61 \pm 0.24)+(0.29 \pm 0.03) \log \left(M_{\star}\right)$, which agrees with previous studies; however, the dispersion $(\sigma=0.15)$ is not significantly lower than that of the $L-Z$ relationships. Because of the low dispersions in these relationships, if an accurate distance is available, the luminosity of a low luminosity galaxy is often a better indicator of metallicity than that derived using certain "strong-line" methods, so significant departures from the $L-Z$ relationships may indicate that caution is prudent in such cases. With these new "direct" metallicities we also revisit the $70 / 160 \mu \mathrm{m}$ color metallicity relationship. Additionally, we examine $\mathrm{N} / \mathrm{O}$ abundance trends with respect to oxygen abundance and $B-V$ color. We find a positive correlation between $\mathrm{N} / \mathrm{O}$ ratio and $B-V$ color for $0.05 \lesssim B-V \lesssim 0.75: \log (\mathrm{N} / \mathrm{O})=(1.18 \pm 0.9) \times(B-V)+(-1.92 \pm 0.08)$, with a dispersion of $\sigma=0.14$, which is in agreement with previous studies.
\end{abstract}

Key words: galaxies: abundances - galaxies: dwarf - galaxies: evolution

Online-only material: color figure

\section{INTRODUCTION}

There is a fundamental relationship between the mass of stars in a galaxy and its metallicity evolution (e.g., Tremonti et al. 2004 , hereafter the $M-Z$ relation). Empirically, this has been observed as a luminosity-metallicity relationship (hereafter the $L-Z$ relation) for low-redshift dwarf galaxies (e.g., Lequeux et al. 1979; Skillman et al. 1989; Lee et al. 2006a, and references therein) and spiral galaxies (e.g., McCall et al. 1985; Garnett \& Shields 1987; Zaritsky et al. 1994; Tremonti et al. 2004, and references therein). This relationship is observed over a range of 10 mag in galaxy optical luminosity (e.g., Zaritsky et al. 1994; Tremonti et al. 2004; Lee et al. 2006a), but the data are relatively sparse at the low luminosity end where the intrinsic faintness of these galaxies makes metallicity determinations more difficult.

\footnotetext{
* Observations reported here were obtained at the MMT Observatory, a joint facility of the University of Arizona and the Smithsonian Institution.
}

The physical driver of the $M-Z$ relation remains under debate. One possibility is that low-mass galaxies are younger, in that they only recently started forming stars (Noeske et al. 2000; Leitner \& Kravtsov 2011). Another is that they have been less efficient at producing metals (Brooks et al. 2007). Many studies favor a different interpretation, where supernova driven winds preferentially expel metals from low-mass galaxies, resulting in a lower effective yield with decreasing mass (e.g., Dekel \& Silk 1986). However, Dalcanton (2007) emphasizes the importance of star formation efficiency as outflows are an insufficient regulator in the absence of depressed star formation. In addition, Dalcanton's calculations show that low effective yields cannot be due to gas infall. Alternatively, Köppen et al. (2007) showed that the $M-Z$ relationship may be observed naturally if a star formation rate (SFR)-dependent, and therefore mass-dependent, stellar initial mass function (IMF) is assumed. Clearly a better understanding of the mass-metallicity relationship at low luminosity remains important to determine how galaxies evolve (e.g., 
see discussion in Moustakas et al. 2012, and references therein). In addition, a well-defined low-luminosity $M-Z$ relationship will provide clues to the source of its measurable scatter. While observational errors play a role, one or more physical processes may be responsible for the remainder. Suggestions for the scatter include variations in the star formation history (e.g., recent starbursts; Contini et al. 2002), variations in stellar surface mass density (Ellison et al. 2008), inflow of metal-poor gas, perhaps triggered by interactions (Lee et al. 2004), and variations in local galaxy density (e.g., Cooper et al. 2008, and references therein). As astronomers examine the interrelationship between chemical abundance measurements, star formation, gas accretion, and gas outflow by measuring the evolution of the $M-Z$ relationship, a secure $M-Z$ relationship for the current epoch is needed for comparison.

Empirical and theoretical oxygen abundance calibrations often introduce bias, further limiting the $M-Z$ relationship (e.g., Yin et al. 2007; Pérez-Montero \& Contini 2009; Moustakas et al. 2010; Berg et al. 2011). Notably, for 53,000 SDSS galaxies, which span 10 orders in the $B$-band magnitude, Tremonti et al. (2004) found a dispersion of 0.16 for their $L-Z$ relationship and 0.10 for their $M-Z$ relationship. Lee et al. (2006a, hereafter L06) were able to extend the mass-metallicity relation lower by 2.5 decades in stellar mass using $4.5 \mu \mathrm{m}$ luminosities for 27 nearby dwarf irregular galaxies. Interestingly, L06 found the dispersion in the near-infrared $L-Z$ relationship to be smaller than the corresponding dispersion in the $B$-band $L-Z$ relationship and nearly identical to that of the $M-Z$ relationship. The smaller dispersion in the near-infrared is not totally unexpected, as NIR luminosities are less sensitive to extinction from dust and variations in SFR. However, the significant but uncertain stochastic effects of asymptotic giant branch (AGB) stars on the total NIR luminosities of low luminosity galaxies must also be considered (see, e.g., Fouesneau \& Lançon 2010; Meidt et al. 2012; Melbourne et al. 2012).

To thoroughly examine the $L-Z$ and $M-Z$ relations, we need a robust sample of galaxies. The Spitzer Local Volume Legacy (LVL) survey ${ }^{11}$ (Dale et al. 2009) covers a volumecomplete sample of 258 galaxies in the local universe with multiwavelength observations spanning the ultraviolet to the radio. The LVL is leveraged by ancillary data including $\mathrm{H} \alpha$ (Kennicutt et al. 2008) and UV (Lee et al. 2011) imaging from the $11 \mathrm{Mpc} \mathrm{H} \alpha$ and Ultraviolet Galaxy Survey (Lee et al. 2011) and the Nearby Galaxy Survey (Gil de Paz et al. 2007). A subsample of the LVL also contains stellar population mapping from the ACS Nearby Galaxy Survey Treasury (Dalcanton et al. 2009), H I mapping from the Very Large Array and GMRT, and optical broadband imaging (Cook et al. 2012; L. van Zee et al. 2012, in preparation) and spectroscopy. However, many of the faintest objects are missing the high-quality optical spectroscopy needed to determine "direct" oxygen-abundance metallicity estimates.

As the $L-Z$ relationship provides both a very strong constraint on theories of galaxy evolution and a tool to better understand galaxies at higher redshifts (Kobulnicky et al. 2003), we are motivated to better characterize the low-luminosity end of the $L-Z$ relationship. Thus, we obtained high-resolution MMT spectroscopy of 42 low-luminosity star-forming galaxies in the Local Volume with the goal of detecting the [O III] $\lambda 4363$ line in order to constrain electron temperature measurements.

\footnotetext{
11 http://www.ast.cam.ac.uk/research/lvls
}

We present our low-luminosity sample in Section 2.2, with spectral observations obtained from the MMT in Section 3.1 and Infrared Array Camera (IRAC) photometry in Section 3.2. Section 4 describes the data reduction, followed by the description of the method used to determine "direct" oxygen abundances in Section 5. Our "Select" sample, compiled from objects with "direct" oxygen abundances and secure distance estimates, is defined in Section 6.1. Using this sample, metallicity is compared to expected trends with $B$-band luminosity, $4.5 \mu \mathrm{m}$ luminosity, and stellar mass in Sections 6.2, 6.3, and 6.4, respectively. N/O relative abundances are discussed in Section 7. In Section 8, we discuss the results of the relationships found in Sections 6.2-6.4, the "young galaxy" hypothesis, and the quality of abundance estimators. Finally, we summarize our conclusions in Section 9. Appendix A presents the strong-line abundances for the low-luminosity LVL galaxies for which we were unable to determine "direct" abundances and Appendix B presents our new "direct" abundances in comparison to the color-temperature-metallicity relationship of Engelbracht et al. (2008).

\section{SAMPLE SELECTION}

\subsection{Spitzer LVL Survey}

LVL is a Spitzer Space Telescope legacy program that combines IRAC and MIPS (Multiband Imaging Photometer) infrared imaging for a complete sample of 258 galaxies for the nearest $11 \mathrm{Mpc}$ of our local universe. These data build upon recent Local Volume galaxy surveys: narrowband $\mathrm{H} \alpha$ (Kennicutt et al. 2008), GALEX ultraviolet (Lee et al. 2011), and Hubble Space Telescope resolved stellar population imaging (Dalcanton et al. 2009). While previous surveys comprehensively cover high surface brightness systems in flux-limited samples, the LVL survey, although also biased toward high surface brightness galaxies, provides a multi-wavelength inventory of a statistically robust, approximately volume-limited sample, which is well suited for studies of dwarf galaxies. By studying the nearby, low-luminosity galaxies, we can increase the dynamic range covered by the luminosity-metallicity and mass-metallicity relationships, which will help to better constrain the slopes.

\subsection{Low-luminosity LVL Sample}

We selected a sample of 42 low-luminosity galaxies in the LVL survey in order to obtain new MMT high-resolution spectra. These low luminosity spirals and dwarf irregulars span a range in distance of $2.5 \leqslant D \leqslant 14.0 \mathrm{Mpc} .^{12}$ The luminosities for this sample range in the near-IR (determined from IRAC Fazio et al. 2004 photometry) from $M_{[4.5]}=-13.1$ to -21.7 , with $B$ band magnitudes of $-10.8 \geqslant M_{B} \geqslant-18.8$. Most of the objects were chosen because they lack "direct" oxygen abundances in the literature, their abundance estimates are dated, or were studied with instruments which were known to have problems.

Although not LVL objects, two additional galaxies were added to the sample (increasing the sample total to 44 objects) because they played a role in motivating this project. Both UGC 4393 and UGC 10818 were identified by Engelbracht et al. (2008) as low metallicity outliers from the global trend of $70 / 160 \mu \mathrm{m}$ color temperature as a function of metallicity. These two galaxies affect the interpretation of the trend for

\footnotetext{
12 Since the inception of the Spitzer LVL program, four galaxies included in the sample have updated distances which place them outside of $11 \mathrm{Mpc}$ (see Dale et al. 2009; Lee et al. 2011).
} 
aromatic emission to weaken below $12+\log (\mathrm{O} / \mathrm{H})=7.9$ in the mid-IR (see, e.g., Engelbracht et al. 2008) and the far-IR (see, e.g., Draine et al. 2007; Engelbracht et al. 2008). Because of the possibility that these objects' oxygen abundances were underestimated using the lower branch of the $R_{23}$ calibration (Pilyugin \& Thuan 2005), they were included in this sample to be re-examined (see discussion in Appendix B). See Table 1 for sample characteristics.

\section{DATA}

\subsection{MMT Spectra}

\subsubsection{Observations}

New spectroscopy was acquired at the MMT in order to achieve high signal-to-noise $(\mathrm{S} / \mathrm{N})$ spectra with the goal of detecting the faint [O III] $\lambda 4363$ auroral line at a strength of $4 \sigma$ or higher. The observations were obtained with the Blue Channel spectrograph (Schmidt et al. 1989) on the UT dates of 2008 October 30-November 1, 2009 June 15-22, and 2010 January 11-12. Sky conditions varied, but contained minimal cloud coverage and approximately arcsecond seeing. A 500 line grating, $1^{\prime \prime}$ slit, and UV-36 blocking filter were used, yielding an approximate dispersion of $1.2 \AA \mathrm{pixel}^{-1}$, a full width at halfmaximum resolution of $\lesssim 3 \AA$, and a wavelength coverage of 3690-6790 А. The sensitivity, resolution, and wavelength coverage of the MMT and Blue Channel spectrograph combination allowed for the measurement of all emission lines relevant to oxygen abundance determinations. Bias frames, flat-field lamp images, and sky flats were taken each night. The latter were primarily necessary due to significant differences between the chip illumination patterns of the sky and the MMT Top Box that houses the "BC" incandescent flat-field lamp. On average, four standard stars from Oke (1990) with spectral energy distributions (SEDs) peaking in the blue and containing minimal absorption were observed throughout the night using a $5^{\prime \prime}$ slit over a range of airmasses. This allows the flux calibration to be determined as a function of airmass. The large slit width mitigates the effects of atmospheric differential refraction and allows accurate measurements of relative fluxes across a large range in wavelength. Note that since we only care about relative abundances, an absolute flux calibration is not critical.

All 44 galaxies had at least one strong $\mathrm{H} \alpha$ brightness peak that was aligned with the $1^{\prime \prime} \times 180^{\prime \prime}$ slit. Typically, three $900 \mathrm{~s}$ exposures $^{13}$ were made with the slit at a fixed position angle which approximated the parallactic angle at the midpoint of the observation and laid across several $\mathrm{H} \alpha$ bright regions when possible. This, in addition to observing the galaxies at airmasses less than 1.5, served to minimize the wavelength-dependent light loss due to differential refraction (Filippenko 1982). A single slit position for each target was deemed sufficient to characterize the global oxygen abundance, as metallicity gradients are observed to be small or non-existent in low-mass galaxies (e.g., Skillman et al. 1989; Kobulnicky \& Skillman 1996, 1997; Lee et al. 2006b; Croxall et al. 2009). Finally, combined helium, argon, and neon arc lamps were observed at each pointing for accurate wavelength calibration. A log of the observations is provided in Table 2. Figure 1 shows the $R$-band continuum and $\mathrm{H} \alpha$ continuum-subtracted images for each galaxy, motivating our slit location choices. The brightest $\mathrm{H} \alpha$ regions observed are

\footnotetext{
13 Some galaxy observations were adjusted to shorter or longer exposures depending on the brightness of the $[\mathrm{O}$ III $] \lambda 4363$ line strength or included additional exposures when the observing program allowed for it; see Table 2.
}

ordered alphabetically by decreasing flux, and the slit positions on the galaxies are shown. The images scale as $60 \times 60 \operatorname{arcsec}$ with north oriented up and east to the left.

\subsubsection{Spectra Reduction}

The MMT observations were processed using ISPEC2D (Moustakas \& Kennicutt 2006), a long-slit spectroscopy data reduction package written in IDL. A master bias frame was created from $\gtrsim 20$ zero second exposures by discarding the highest and lowest value at each pixel and taking the median. Master sky and dome flats were similarly constructed after normalizing the counts in the individual images. Those calibration files were then used to bias-subtract, flat-field, and illumination-correct the raw data frames. Dark current was measured to be an insignificant $\sim 1 e^{-}$per pixel per hour and was not corrected for.

Misalignment between the trace of the light in the dispersion direction and the orientation of the CCD detector was rectified via the mean trace of the standard stars for each night, providing alignment to within a pixel across the detector. A twodimensional sky subtraction was performed using individually selected sky apertures, followed by a wavelength calibration applied from the HeArNe comparison lamps taken at the same telescope pointing. Airmass-dependent atmospheric extinction and reddening were corrected for using the standard Kitt Peak extinction curve (Crawford \& Barnes 1970).

For each galaxy, the multiple sub-exposures were combined, eliminating cosmic rays in the process. The resulting images were then flux calibrated using the sensitivity curve derived from the standard star observations taken throughout a given night. Finally, the trace fit to the strongest continuum source in the slit was used to extract the galaxy emission within apertures that encompassed $\gtrsim 99 \%$ of the light. Figure 2 shows a sample of four of the resulting one-dimensional spectra extracted for galaxies that had significant $[\mathrm{O}$ III $] \lambda 4363$ detections. The inset windows display a narrower spectral range to emphasize the $[\mathrm{O}$ III $] \lambda 4363$ strength. This sample does not feature the best spectra from our sample, but rather galaxies are ordered by ionizing radiation field strength from highest to lowest as given by the [O III] $\lambda 5007 /[\mathrm{O}$ II $] \lambda 3727$ ratio, highlighting the variation within the sample.

\subsection{Photometry}

To better characterize our low-luminosity sample, absolute magnitudes in several different bands were obtained. Here, we describe their origin and reference their subsequent use. $M_{B}$ values were determined by $L$. van Zee et al. (2012, in preparation) using photometry from apertures matched to the infrared LVL photometry (unless otherwise noted). Optical photometry for the entire LVL sample is given in Cook et al. (2012), whereas L. van Zee et al. (2012, in preparation) focus on the analysis of colors and EW gradients of dwarf galaxies. The data are used to examine the optical luminosity-metallicity relationship (see Section 6.2).

$M_{[4.5]}$ values from the $4.5 \mu \mathrm{m}$ IRAC photometry presented in Dale et al. (2009) were calculated using

$$
M_{[4.5]}=-2.5 \log \frac{F_{[4.5]}(d / 10)^{2}}{179.7},
$$

where $F_{[4.5]}$ is the $4.5 \mu \mathrm{m}$ flux in Janskys, $d$ is the distance in parsecs, and 179.7 is the zero point flux in Janskys for the $4.5 \mu \mathrm{m}$ IRAC band (Reach et al. 2005). Distances are taken from the literature, as described in Table 1, and assumed 
Table 1

(Continued)

\begin{tabular}{|c|c|c|c|c|c|c|c|c|c|c|c|c|}
\hline Galaxy & $\begin{array}{l}\text { R.A. } \\
\text { (J2000) }\end{array}$ & $\begin{array}{l}\text { Decl. } \\
\text { (J2000) }\end{array}$ & $\begin{array}{l}F_{[4.5]} \\
(\mathrm{mJy})\end{array}$ & $\begin{array}{c}F_{K_{S}} \\
(\mathrm{mJy})\end{array}$ & $\begin{array}{c}D \\
(\mathrm{Mpc})\end{array}$ & $\begin{array}{c}\text { Reference } \\
\text { Method }\end{array}$ & $\begin{array}{c}M_{B} \\
(\mathrm{mag})\end{array}$ & $\begin{array}{l}M_{[4.5]} \\
(\mathrm{mag})\end{array}$ & $\begin{array}{c}M_{K_{S}} \\
(\mathrm{mag})\end{array}$ & $\begin{array}{l}(B-V)_{0} \\
(\mathrm{mag})\end{array}$ & $\begin{array}{c}\log L_{[4.5]} \\
\left(L_{\odot}\right)\end{array}$ & $\begin{array}{c}\log M_{\star} \\
\left(M_{\odot}\right)\end{array}$ \\
\hline NGC 4656 & $12: 43: 57.7$ & $32: 10: 05$ & 70.5 & $135 \pm 14$ & 8.6 & $6, \mathrm{v}$ (flow) & $-18.75 \pm 0.51^{\mathrm{a}}$ & $-21.15 \pm 0.61$ & $-20.44 \pm 0.53$ & $0.42^{\mathrm{c}}$ & $9.79 \pm 0.61$ & $9.04 \pm 0.61$ \\
\hline UGC 8201 & $13: 06: 24.5$ & $67: 42: 28$ & 9.09 & $37.3 \pm 6.3$ & $4.57 \pm 0.40$ & $3, \operatorname{trgb}$ & $-15.17 \pm 0.44$ & $-17.56 \pm 0.60$ & $-17.67 \pm 0.45$ & $0.24 \pm 0.04$ & $8.36 \pm 0.60$ & $7.82 \pm 0.60$ \\
\hline UGC 8245 & $13: 08: 35.2$ & $78: 56: 14$ & 5.98 & $20.0 \pm 4.3$ & 3.64 & $6, \mathrm{v}$ (flow) & $-13.67 \pm 0.50$ & $-16.61 \pm 0.61$ & $-16.50 \pm 0.51$ & $0.47 \pm 0.04$ & $7.98 \pm 0.61$ & $7.53 \pm 0.61$ \\
\hline UGC 8508 & 13:30:44.1 & $54: 54: 40$ & 4.87 & $13.9 \pm 4.2$ & $2.58 \pm 0.03$ & $4, \operatorname{trgb}$ & $-13.03 \pm 0.07$ & $-15.64 \pm 0.35$ & $-15.36 \pm 0.13$ & $0.37 \pm 0.03$ & $7.59 \pm 0.35$ & $7.00 \pm 0.35$ \\
\hline UGC 8638 & $13: 39: 19.2$ & $24: 46: 36$ & 5.01 & $15.2 \pm 4.6$ & $4.27 \pm 0.34$ & $5, \operatorname{trgb}$ & $-13.77 \pm 0.40$ & $-16.77 \pm 0.53$ & $-16.55 \pm 0.41$ & $0.47 \pm 0.04$ & $8.04 \pm 0.53$ & $7.57 \pm 0.53$ \\
\hline UGC 8837 & $13: 54: 45.7$ & $53: 54: 03$ & 10.1 & $26.5 \pm 7.1$ & 8.3 & $2, \mathrm{bs}$ & $-15.92 \pm 0.51^{\mathrm{a}}$ & $-18.97 \pm 0.61$ & $-18.59 \pm 0.52$ & $0.42^{\mathrm{c}}$ & $8.92 \pm 0.61$ & $8.41 \pm 0.61$ \\
\hline NGC 5477 & $14: 05: 33.1$ & $54: 27: 39$ & 5.17 & $22.0 \pm 4.5$ & 7.7 & 2 , bs & $-15.22 \pm 0.51^{\mathrm{a}}$ & $-18.08 \pm 0.61$ & $-18.23 \pm 0.51$ & $0.34^{\mathrm{c}}$ & $8.56 \pm 0.61$ & $8.15 \pm 0.61$ \\
\hline UGC 9405 & $14: 35: 24.4$ & $57: 15: 19$ & 3.97 & $12.9 \pm 5.0$ & $8.02 \pm 0.74$ & 8, bs & $-14.97 \pm 0.47^{\mathrm{a}}$ & $-17.88 \pm 0.58$ & $-17.74 \pm 0.47$ & $0.68^{\mathrm{d}}$ & $8.48 \pm 0.58$ & $7.97 \pm 0.58$ \\
\hline UGC 10818 & $17: 19: 42$ & $61: 18: 47$ & 4.65 & & 56 & $1, \mathrm{~h}$ (flow) & $-18.59 \pm 0.50$ & $-20.96 \pm 0.51^{\mathrm{b}}$ & & & $9.72 \pm 0.51$ & $9.45 \pm 0.51$ \\
\hline KKH 98 & $23: 45: 34.3$ & $38: 43: 00$ & 1.56 & $7.56 \pm 3.09$ & $2.45 \pm 0.04$ & $4, \operatorname{trgb}$ & $-11.10 \pm 0.16$ & $-14.29 \pm 0.36$ & $-14.58 \pm 0.11$ & $0.20 \pm 0.13$ & $7.05 \pm 0.36$ & $6.72 \pm 0.36$ \\
\hline
\end{tabular}

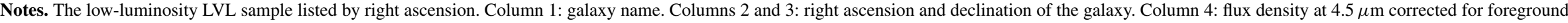

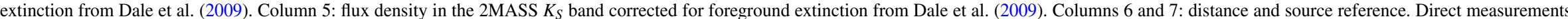

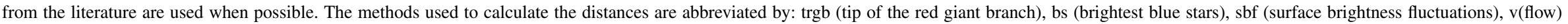

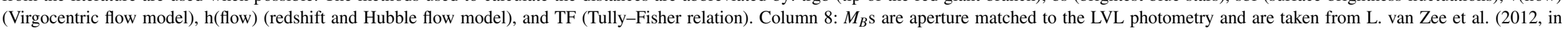

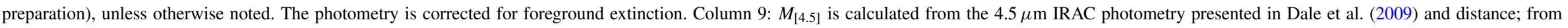

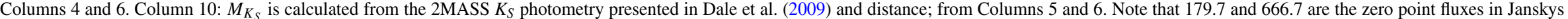

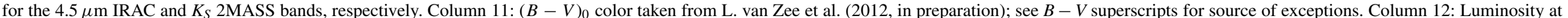

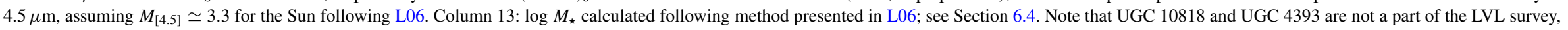
but have been included here for completeness (See Section 2.2)

${ }^{a}$ Kennicutt et al. (2008); photometry is extinction corrected.

b Engelbracht et al. (2008), photometry is extinction corrected.

${ }^{c}$ de Vaucouleurs et al. (1991); photometry is extinction corrected and $k$-corrected.

${ }^{\mathrm{d}}$ Calculated from $g-r$ colors, available on SDSS.org, with foreground extinction correction and 1 st order $k_{\text {filter }}$ extinction correction.

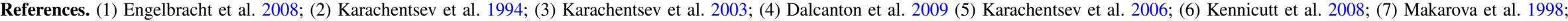

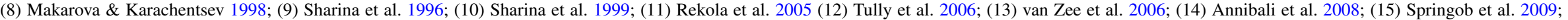

(16) A. Aloisi, 2011 private communication. 
Table 2

Observing Log

\begin{tabular}{|c|c|c|c|c|c|}
\hline $\begin{array}{l}\text { Slit } \\
\text { Position }\end{array}$ & $\begin{array}{l}\text { R.A. } \\
(2000)\end{array}$ & $\begin{array}{l}\text { Decl. } \\
(2000)\end{array}$ & $\begin{array}{l}\text { P.A. } \\
\text { (deg) }\end{array}$ & Run & $\begin{array}{c}T_{\text {int }} \\
(\mathrm{s})\end{array}$ \\
\hline UGC 521-A & $00: 51: 11.9$ & $12: 01: 34$ & -55.71 & Nov08 & $3 \times 900$ \\
\hline UGC 521-B & $00: 51: 12.1$ & 12:01:31 & -55.71 & Nov08 & $2 \times 900$ \\
\hline UGC 695-E & 01:07:46.5 & 01:03:53 & 29.44 & Jan10 & $4 \times 900$ \\
\hline NGC 0404-A & 01:09:26.0 & $35: 43: 00$ & -76.70 & Jan10 & $3 \times 600$ \\
\hline UGC 1056-A & $01: 28: 47.3$ & $16: 41: 16$ & 45.19 & Jan 10 & $3 \times 900$ \\
\hline UGC 1056-B & $01: 28: 47.5$ & $16: 41: 21$ & 45.19 & Jan 10 & $4 \times 900$ \\
\hline UGC 1176-A & 01:40:11.9 & $15: 54: 46$ & 42.14 & Jan 10 & $4 \times 900$ \\
\hline UGC 784-B & $02: 01: 16.5$ & $28: 50: 06$ & 52.76 & Jan 10 & $4 \times 900$ \\
\hline UGC 784-A & $02: 01: 17.5$ & $28: 50: 16$ & 52.76 & Jan 10 & $4 \times 1200$ \\
\hline UGC 2716-A & $03: 24: 07.2$ & $17: 45: 11$ & 63.24 & Jan10 & $3 \times 900$ \\
\hline KKH 037-A & $06: 47: 43.1$ & $80: 07: 27$ & -176.05 & Jan10 & $1 \times 1800$ \\
\hline NGC 2537-A & $08: 13: 13.0$ & $45: 59: 39$ & -94.27 & Jan10 & $3 \times 900$ \\
\hline NGC 2537-B & $08: 13: 13.3$ & $45: 59: 39$ & -94.27 & Jan10 & $3 \times 900$ \\
\hline UGC 4278-B & 08:14:00.2 & $45: 42: 58$ & -128.00 & Oct08 & $3 \times 1800$ \\
\hline UGC 4278-A & $08: 14: 00.0$ & $45: 42.57$ & -128.00 & Oct08 & $3 \times 1800$ \\
\hline NGC 2552-A & $08: 19: 17.1$ & 50:00:14 & -120.00 & Oct08 & $3 \times 1200$ \\
\hline UGC 4393-B & $08: 26: 05.3$ & $45: 58: 10$ & -124.65 & Jan10 & $3 \times 900$ \\
\hline UGC 4393-C & $08: 26: 01.5$ & $45: 47: 43$ & -124.65 & Jan10 & $3 \times 900$ \\
\hline CGCG 035-007-A & $09: 34: 44.4$ & $06: 25: 31$ & 42.78 & $\operatorname{Jan} 10$ & $3 \times 900$ \\
\hline UGC 5139-A & 09:40:16.0 & $71: 10: 06$ & -140.00 & Nov08 & $4 \times 1200$ \\
\hline IC 559-A & $09: 44: 42.9$ & $09: 36: 54$ & -64.15 & Jan10 & $4 \times 900$ \\
\hline UGC 5272-A & 09:50:22.3 & $31: 29: 15$ & -80.51 & Oct08 & $3 \times 600$ \\
\hline UGC 5340-A & 09:56:46.8 & $28: 50: 10$ & -75.61 & Jan10 & $4 \times 900$ \\
\hline UGC 5423-A & $10: 05: 28.7$ & $70: 22: 05$ & 127.00 & Jan10 & $3 \times 900$ \\
\hline UGC 5423-B & $10: 05: 32.1$ & $70: 21: 52$ & 127.00 & Jan10 & $3 \times 900$ \\
\hline UGC 5672-A & $10: 28: 21.1$ & 22:34:05 & -57.80 & $\operatorname{Jan} 10$ & $4 \times 900$ \\
\hline UGC 5692-A & $10: 30: 34.8$ & 70:37:11 & -147.53 & $\operatorname{Jan} 10$ & $4 \times 900$ \\
\hline UGC 5797-A & $10: 39: 25.0$ & 01:43:00 & -4.17 & Jan10 & $3 \times 900$ \\
\hline UGC 5923-A & $10: 49: 07.5$ & 06:55:08 & 20.00 & Jan10 & $5 \times 600$ \\
\hline NGC 3738-A & $11: 35: 46.8$ & $54: 31: 32$ & 93.73 & Jun09 & $4 \times 900$ \\
\hline NGC 3738-B & $11: 35: 48.2$ & $54: 31: 31$ & 93.73 & Jun09 & $4 \times 900$ \\
\hline NGC 3741-A & $11: 36: 05.9$ & $45: 17: 00$ & 101.03 & Jun09 & $3 \times 1200$ \\
\hline UGC 6782-A & $11: 48: 57.2$ & $23: 50: 32$ & 64.55 & Jun09 & $3 \times 1200$ \\
\hline UGC 6817-A & $11: 50: 52.9$ & $38: 52: 52$ & 93.87 & Jun09 & $3 \times 1200$ \\
\hline UGC 6900-A & $11: 55: 36.2$ & $31: 31: 19$ & 81.43 & Jun09 & $3 \times 1200$ \\
\hline UGC 4163-A & $12: 12: 09.4$ & $36: 09: 59$ & 87.48 & Jun09 & $3 \times 1200$ \\
\hline CGCG 269-049-A & $12: 15: 46.6$ & $52: 23: 14$ & -187.93 & Jan10 & $4 \times 900$ \\
\hline UGC 7577-A & $12: 27: 42.8$ & 43:29:06 & 100.00 & Jun09 & $3 \times 1200$ \\
\hline NGC 4449- C & $12: 28: 14.5$ & 44:07:13 & 75.00 & Jun09 & $3 \times 600$ \\
\hline NGC 4449- B & $12: 28: 14.1$ & 44:07:12 & 75.00 & Jun09 & $3 \times 600$ \\
\hline NGC 4449-A & $12: 28: 13.9$ & 44:07:10 & 75.00 & Jun09 & $3 \times 600$ \\
\hline UGC 7599-A & $12: 28: 27.2$ & $37: 14: 16$ & 86.11 & Jun09 & $3 \times 1500$ \\
\hline UGC 7605-A & $12: 28: 38.4$ & $35: 43: 15$ & 89.00 & Jun09 & $5 \times 1200$ \\
\hline UGC 7639-A & $12: 29: 54.6$ & $47: 31: 40$ & 95.77 & Jun09 & $2 \times 1200,1 \times 600$ \\
\hline NGC 4656-A & $12: 43: 56.6$ & $32: 10: 12$ & -79.80 & Jan10 & $3 \times 900$ \\
\hline UGC 8201-A & 13:06:17.4 & $67: 42: 08$ & 120.00 & Jun09 & $3 \times 1200$ \\
\hline UGC 8245-A & 13:08:41.0 & $78: 56: 22$ & 150.00 & Jun09 & $3 \times 1200$ \\
\hline UGC 8508-A & $13: 30: 44.5$ & $54: 54: 24$ & 90.09 & Jun09 & $2 \times 1200,1 \times 900$ \\
\hline UGC 8638-A & $13: 39: 19.3$ & $24: 46: 28$ & 69.76 & Jun09 & $3 \times 1200$ \\
\hline UGC 8638-B & $13: 39: 20.5$ & $24: 46: 33$ & 69.76 & Jun09 & $3 \times 1200$ \\
\hline UGC 8837-A & $13: 54: 40.5$ & 53:53:09 & 123.33 & Jun09 & $3 \times 900$ \\
\hline NGC 5477-C & $14: 05: 32.9$ & $54: 27: 41$ & 99.00 & Jun09 & $3 \times 900$ \\
\hline NGC 5477-A & $14: 05: 33.4$ & $54: 27: 41$ & 99.00 & Jun09 & $3 \times 900$ \\
\hline UGC 9405-A & $14: 35: 25.9$ & $57: 15: 29$ & 125.00 & Jun09 & $4 \times 1200$ \\
\hline UGC 10818-A & $17: 19: 41.1$ & $61: 18: 31$ & -180.00 & Jun09 & $3 \times 900$ \\
\hline ККН 098-А & $23: 45: 33.5$ & $38: 43: 15$ & -110.00 & Jan10 & $3 \times 1800$ \\
\hline
\end{tabular}

Notes. The low-luminosity LVL sample observing log. Galaxy name and $\mathrm{H}$ II region label are listed in Column 1 . The right ascension and declination of the individual $\mathrm{H}$ II regions are given in units of hours, minutes, and seconds, and degrees, arcminutes, and arcseconds, respectively. The position angle (P.A.) gives the rotation of the slit counterclockwise from north. Note that the R.A. and decl. positions for "A" regions are accurate and denote the center slit placement. However, "B" and "C" regions only have estimated R.A. and decl. positions, as these are just extra extractions along the same slit that is aligned through "A." 

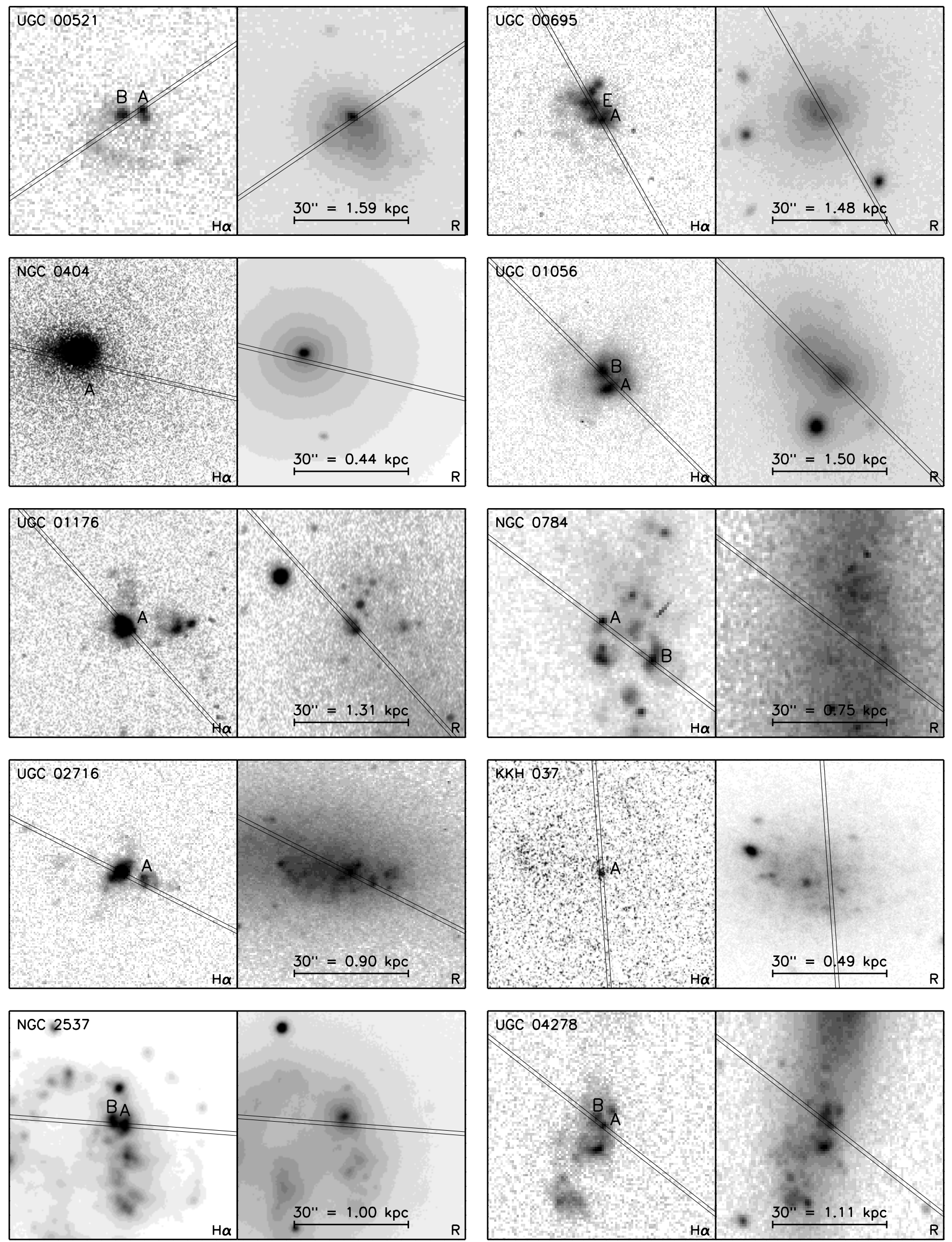

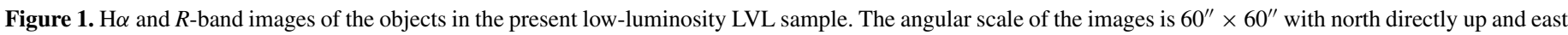

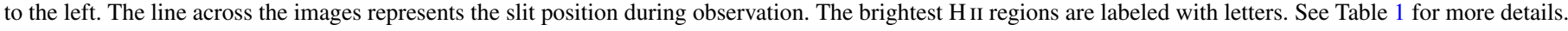



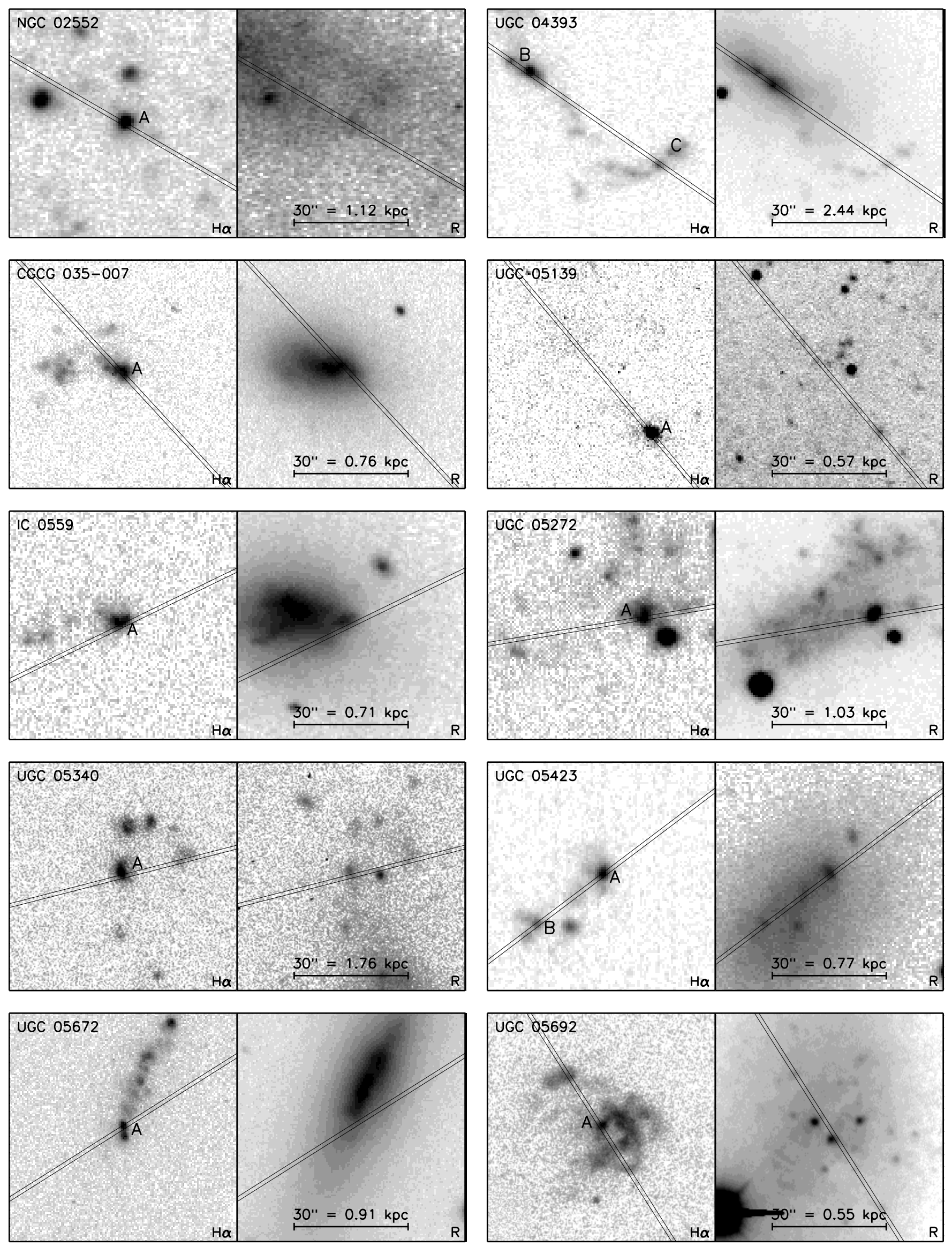

Figure 1. (Continued) 

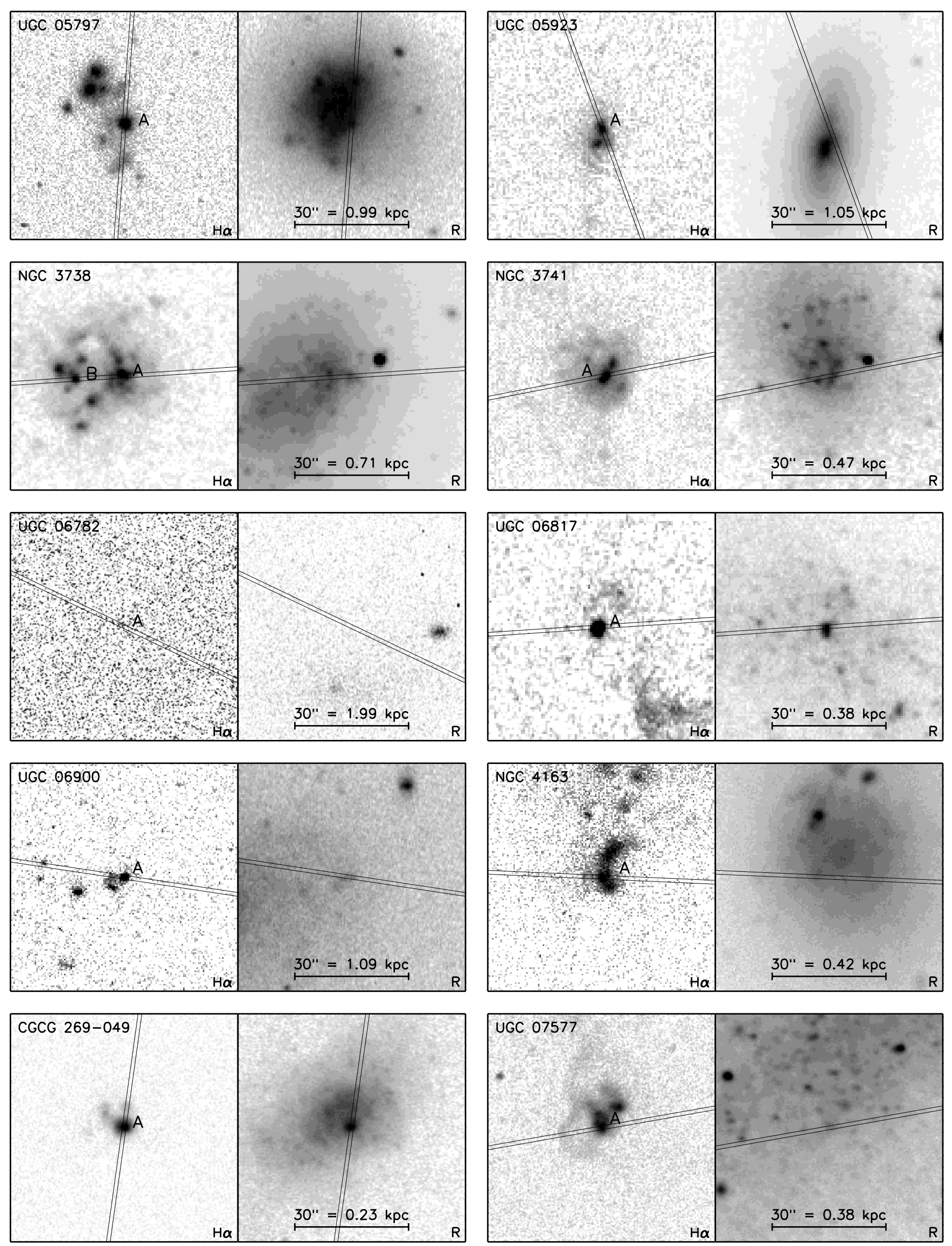

Figure 1. (Continued) 

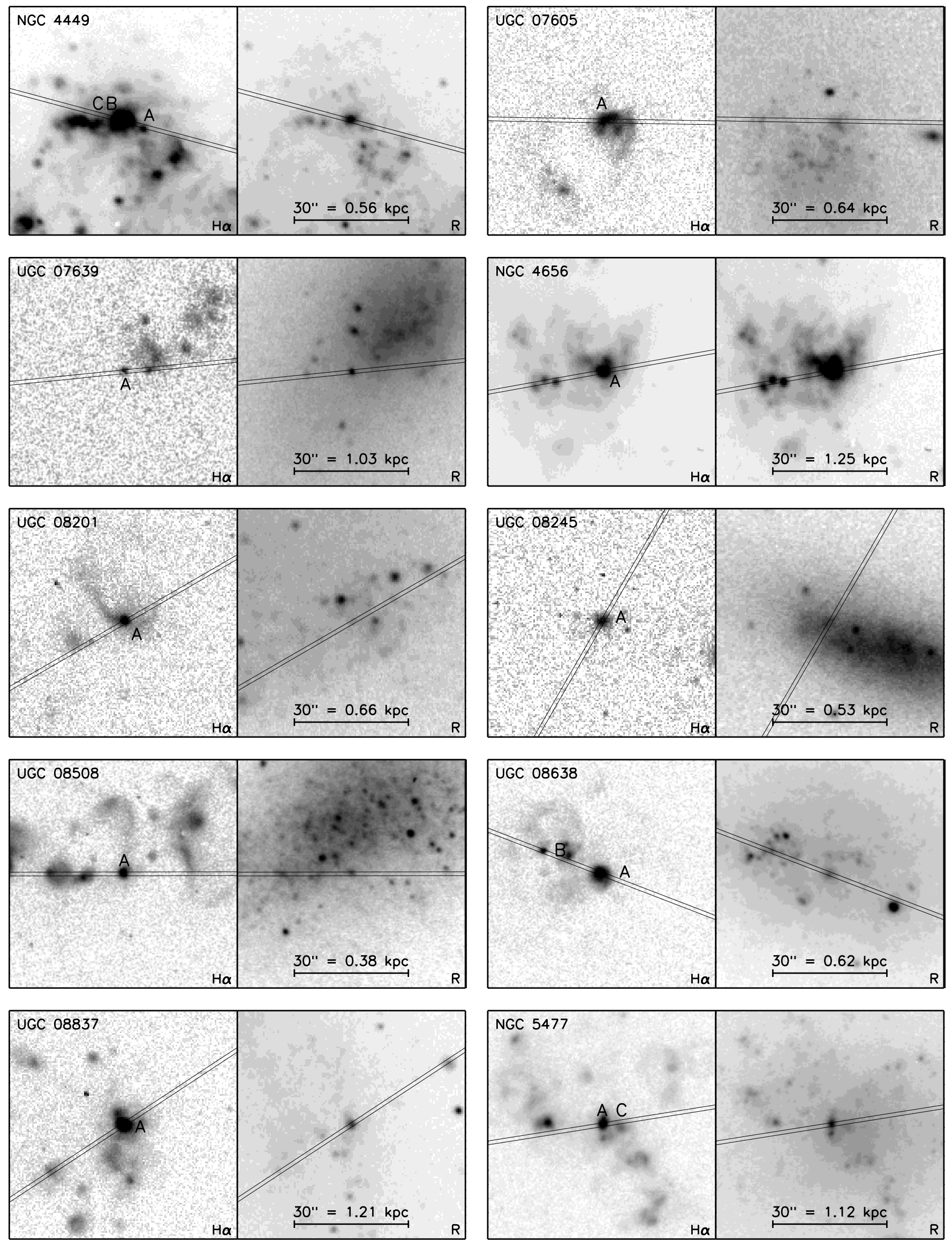

Figure 1. (Continued) 

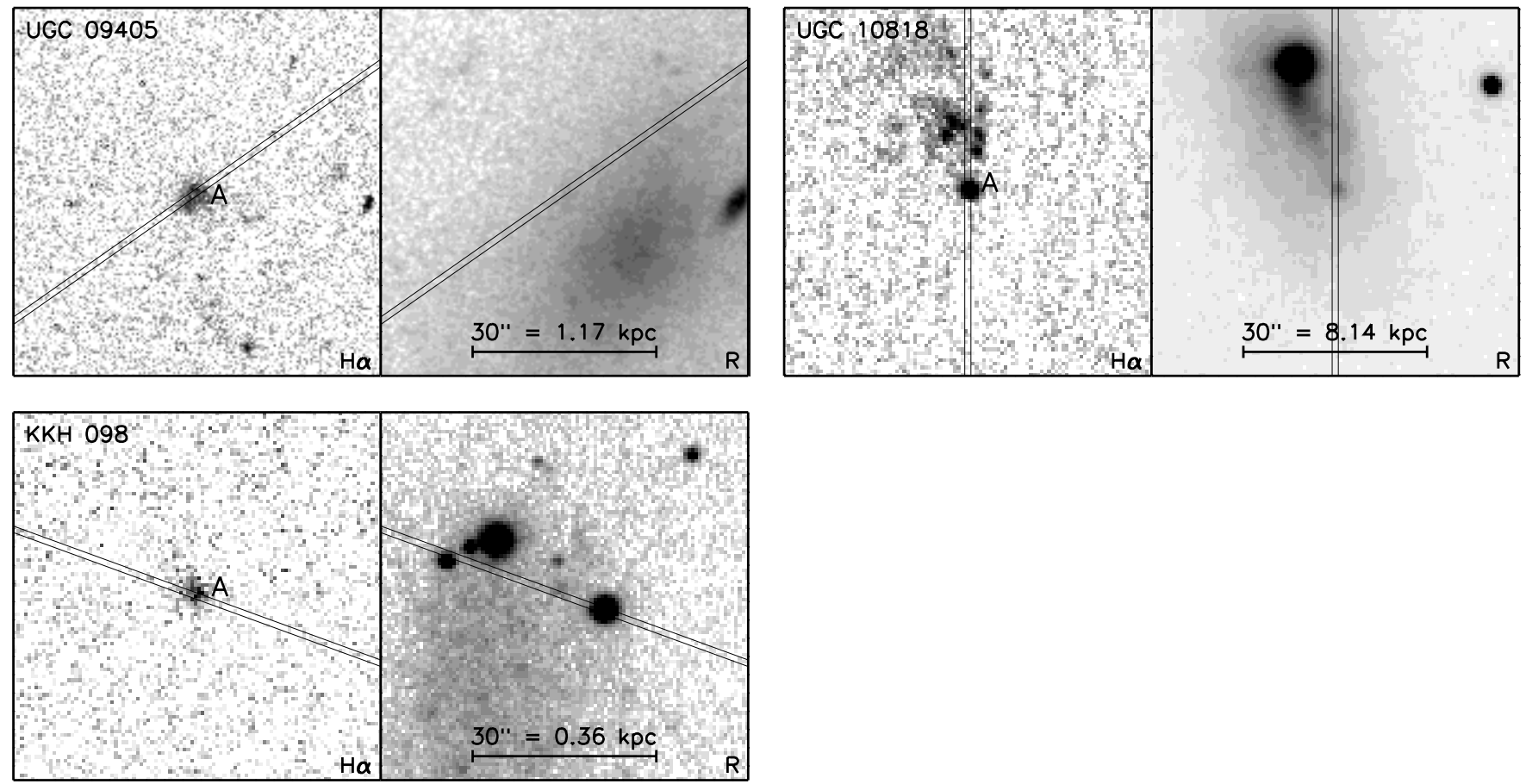

Figure 1. (Continued)

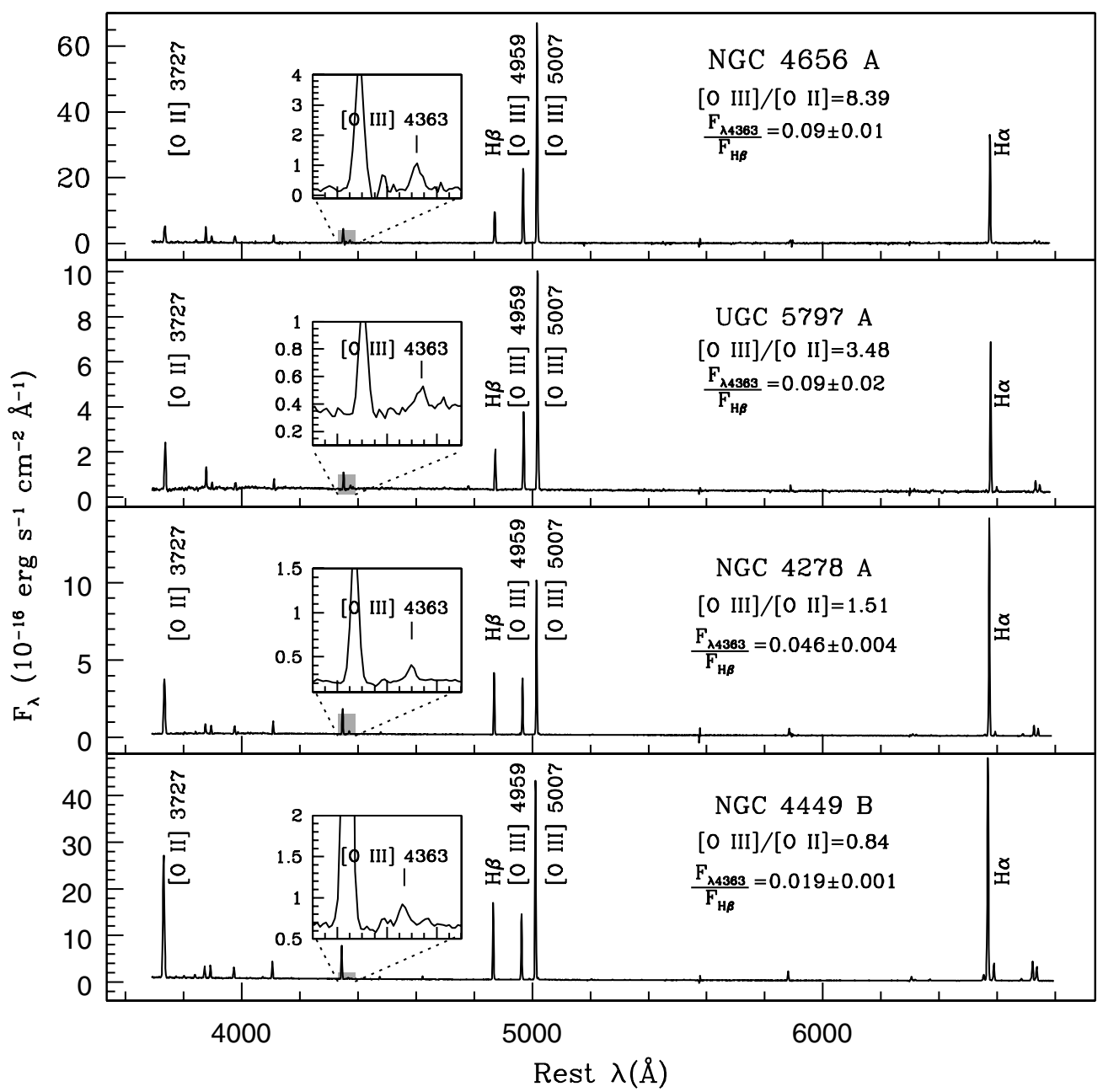

Figure 2. Four sample spectra representative of the low-luminosity LVL sample presented in this paper. The full spectral range of these high-quality, high signal-to-noise observations is shown. The inset windows expand the region around the intrinsically faint [O $\mathrm{III}] \lambda 4363$ line used to determine $T_{\mathrm{e}}$. Note that the much stronger line blueward of [O III] $\lambda 4363$ is $\mathrm{H} \gamma \lambda 4340$. These spectra demonstrate the range in ionization field strength seen for this sample, ranging from low ionization in the bottom panel (NGC 5477) to high ionization in the top panel (UGC 4656). 
to have $10 \%$ uncertainty where none was provided. IRAC calibration uncertainties are $5 \%-10 \%$ for the $4.5 \mu \mathrm{m}$ data. Later, in Section 6.4, we use these $M_{[4.5]}$ magnitudes to analyze the NIR luminosity-metallicity relationship. Similarly, $M_{K_{S}}$ values were determined by Dale et al. (2009) from 2MASS imaging, where 666.7 is the zero point flux in Janskys for the 2MASS $K_{S}$ band. Although 2MASS $F_{K_{S}}$ values are available for those objects for which Dale et al. (2009) do not provide $K_{S}$ magnitudes, we choose not to use them. The small apertures used in the 2MASS extraction produce unexpectedly faint magnitudes for smaller galaxies when compared to similar extractions from IRAC 3.6 and $4.5 \mu \mathrm{m}$ data (see, e.g., Figures 4 and 5 in Dale et al. 2009), and so may not be terribly accurate for our sample. The $K_{S}$ magnitudes were used to determine stellar masses in Section 6.4.

Finally, $V$-band magnitudes were needed to calculate $B-V$ colors (see Table 1). When available, $M_{V}$ values were provided by $\mathrm{L}$. van Zee et al. (2012, in preparation), using the LVL elliptical aperture. In other cases, values are taken from de Vaucouleurs et al. (1991) or are determined using $g$ - and $r$-band photometry available from the Sloan Digital Sky Survey (SDSS; York et al. 2000). The SDSS values are then used to estimate the $B-V$ color following Jester et al. (2005):

$$
B-V=\frac{(g-r)+0.22}{1.02} \text {. }
$$

The available $M_{B}, M_{[4.5]}$, and $B-V$ colors and references for this sample are listed in Table 1 . Note that the main source of uncertainty in these magnitudes lies in the distance determinations. Eight of the objects in our sample have distance errors of approximately 10\%. Furthermore, 20 of the 44 objects in our sample do not have uncertainties associated with their distance determinations. For these objects we used an uncertainty of $10 \%$, which may be an underestimate for some of them. The distance uncertainties tend to dominate over the photometric uncertainties.

\section{NEBULAR ABUNDANCE ANALYSIS}

\subsection{Emission Line Measurements}

Emission line strengths were measured using standard methods available within IRAF. ${ }^{14}$ In particular, the SPLOT routine was used to analyze the extracted one-dimensional spectra and to fit Gaussian profiles to emission lines to determine their integrated fluxes. Special attention was paid to the Balmer lines, which are sometimes located in troughs of significant underlying stellar absorption. The $\mathrm{H} \alpha$ emission lines typically had equivalent widths of $\sim 350 \AA$, large enough that the underlying absorption was not a concern. Even for those $\mathrm{H} \alpha$ emission lines with lower EWs, the underlying absorption was negligible. This was often not the case for $\mathrm{H} \beta$ and the lower equivalent width Balmer lines. The $\mathrm{H} \beta$ absorption EWs for our sample range from 1 to $8 \AA$. These values are typical of local low-luminosity galaxies, with the majority having $\mathrm{H} \beta$ absorption EWs between $0 \AA$ and $5 \AA$ (see, e.g., Figure 6 in Berg et al. 2011). For the bluer Balmer lines, a multiple component fit was used in which the absorption was fit by a broad, negative Lorentzian profile and the emission was fit by a narrow, positive Gaussian profile. To ensure a proper fit of the [O III] $\lambda 4363$ line, $\mathrm{H} \gamma$ was first fit by

\footnotetext{
14 IRAF is distributed by the National Optical Astronomy Observatory, which is operated by the Association of Universities for Research in Astronomy, Inc., under cooperative agreement with the National Science Foundation.
}

a Gaussian profile, then [O III] $\lambda 4363$ was forced to be fit to the same line profile with the assumption that the profile widths of these two neighboring lines should be the same.

Note that we chose to fit the underlying Balmer absorption with Lorentzian profiles, as opposed to using stellar population synthesis continuum fitting common in many studies (e.g., Tremonti et al. 2004). Given the large equivalent widths of the Balmer emission lines, the differences between the two methods are negligible, and the Lorentzian profiles have the advantage of requiring no additional assumptions. Most importantly, for spectra dominated by young stars, at signal-to-noise values typical of our spectra, population synthesis models may not provide a unique solution. There are also very large variations in the population synthesis models for young ages, with large uncertainties in how the Wolf-Rayet phase, stellar winds, rotation, and other parameters are treated. Since mass loss and mixing processes in stellar evolution are still poorly understood, stellar phases, like Wolf-Rayet stars or red supergiants, are particularly affected by such uncertainties (Leitherer \& Ekstrom 2011). Later phases, like AGB stars, are covered only crudely in models or not at all, pushing parameters into regimes that are not properly calibrated. When discrepancies between models are found, they can usually be attributed to different intrinsic input parameters and/or treatment of these aberrant stellar evolutionary phases (Vázquez \& Leitherer 2005; Conroy \& Gunn 2010). By not using the models to fit our continuum, we avoid the uncertainties associated with these implicit assumptions.

The errors of the flux measurements were approximated using

$$
\sigma_{\lambda} \approx \sqrt{(2 \times \sqrt{N} \times \mathrm{rms})^{2}+\left(0.02 \times F_{\lambda}\right)^{2}}
$$

where $N$ is the number of pixels spanning the Gaussian profile fit to the narrow emission lines. The rms noise in the continuum was taken to be the average of the rms on each side of an emission line. For weak lines, whose uncertainty is dominated by error from the continuum subtraction, the rms term determines the approximate uncertainty. For the lines with flux measurements much stronger than the rms noise of the continuum (usually the $\mathrm{H} \alpha$ lines and often the [O III $] \lambda \lambda 4959,5007$ doublet), the error is dominated by flux calibration and de-reddening uncertainties. In this case, a minimum uncertainty of $2 \%$ was assumed, and the right-hand term above dominates the uncertainty estimate. Thirty-one of the 44 galaxies in our sample were measured to have [O III] $\lambda 4363$ line strengths $>4 \sigma$. The measured [O III] $\lambda 4959 / \lambda 5007$ ratios match theoretical expectations within the errors, supporting our error estimates and the assumption that the continuum subtraction dominates the uncertainties for the weak lines. For all the objects in the present sample, flux line strengths and corresponding errors are listed in Table 3. We concentrate the rest of our analysis on the objects for which direct electron temperature and chemical abundance determinations can be made. An analysis of the remaining spectra using strong-line methods is reported in Appendix A.

\subsection{Reddening Corrections}

The relative intensities of the Balmer lines are nearly independent of both density and temperature, so they can be used to solve for the reddening. The MMT spectra were de-reddened using the reddening law of Cardelli et al. (1989), parameterized by $A_{V}=3.1 E(B-V)$, where the extinction, $A_{1}(\lambda)$, was 
Table 3

Emission-Line Intensities and Equivalent Widths for Low-luminosity LVL Galaxies

\begin{tabular}{|c|c|c|c|c|c|c|c|c|c|c|}
\hline \multirow{3}{*}{ Ion } & \multicolumn{10}{|c|}{$I(\lambda) / I(\mathrm{H} \beta)$} \\
\hline & UGC & UGC & UGC & UGC & UGC & NGC & NGC & UGC & KKH & NGC \\
\hline & $521 \mathrm{~A}$ & $695 \mathrm{E}$ & $1056 \mathrm{~A}$ & $1056 \mathrm{~B}$ & $1176 \mathrm{~A}$ & $784 \mathrm{~A}$ & $784 \mathrm{~B}$ & $2716 \mathrm{~A}$ & $037 \mathrm{~A}$ & $2537 \mathrm{~A}$ \\
\hline [O II] $\lambda 3727$ & $1.59 \pm 0.03$ & $3.25 \pm 0.07$ & $3.32 \pm 0.13$ & $2.80 \pm 0.06$ & $2.27 \pm 0.05$ & $2.19 \pm 0.06$ & $2.61 \pm 0.07$ & $2.08 \pm 0.04$ & $6.51 \pm 0.08$ & $3.42 \pm 0.07$ \\
\hline He I $\lambda 3820$ & & & $\ldots$ & $\ldots$ & & & & & $\ldots$ & $\ldots$ \\
\hline H9 $\lambda 3835$ & $0.07 \pm 0.02$ & $0.11 \pm 0.01$ & $\ldots$ & $\ldots$ & $0.07 \pm 0.01$ & $0.04 \pm 0.01$ & $0.07 \pm 0.01$ & $0.08 \pm 0.02$ & $\ldots$ & $\ldots$ \\
\hline$[\mathrm{Ne}$ III] $\lambda 3868$ & $0.33 \pm 0.02$ & $0.19 \pm 0.01$ & $0.27 \pm 0.04$ & $0.30 \pm 0.02$ & $0.28 \pm 0.01$ & $0.33 \pm 0.01$ & $0.29 \pm 0.01$ & $0.38 \pm 0.02$ & $\ldots$ & $0.15 \pm 0.01$ \\
\hline He I+H8 $\lambda 3889$ & $0.17 \pm 0.02$ & $0.25 \pm 0.01$ & $0.17 \pm 0.03$ & $0.24 \pm 0.02$ & $0.19 \pm 0.01$ & $0.18 \pm 0.01$ & $0.23 \pm 0.01$ & $0.26 \pm 0.01$ & $\ldots$ & $0.17 \pm 0.01$ \\
\hline$[\mathrm{Ne}$ III]+H7 $\lambda 3968$ & $0.34 \pm 0.02$ & $0.36 \pm 0.01$ & $0.37 \pm 0.02$ & $0.54 \pm 0.02$ & $0.59 \pm 0.01$ & $0.20 \pm 0.01$ & $0.51 \pm 0.01$ & $0.32 \pm 0.01$ & $\ldots$ & $0.30 \pm 0.01$ \\
\hline Не г $\lambda 4026$ & $\ldots$ & $\ldots$ & $\ldots$ & $\ldots$ & $\ldots$ & $\ldots$ & $\ldots$ & $\ldots$ & $\ldots$ & $0.025 \pm 0.003$ \\
\hline [S II] $\lambda 4068$ & $\ldots$ & $0.03 \pm 0.02$ & $\ldots$ & $\ldots$ & $\ldots$ & $\ldots$ & $\ldots$ & $\ldots$ & $\ldots$ & \\
\hline $\mathrm{H} \delta \lambda 4101$ & $0.26 \pm 0.02$ & $0.29 \pm 0.02$ & $0.27 \pm 0.02$ & $0.26 \pm 0.01$ & $0.26 \pm 0.01$ & $0.264 \pm 0.006$ & $0.59 \pm 0.01$ & $0.27 \pm 0.01$ & $\ldots$ & $0.27 \pm 0.01$ \\
\hline $\mathrm{H} \gamma \lambda 4340$ & $0.48 \pm 0.01$ & $0.53 \pm 0.01$ & $0.49 \pm 0.01$ & $0.50 \pm 0.01$ & $0.46 \pm 0.01$ & $0.460 \pm 0.009$ & $0.48 \pm 0.01$ & $0.51 \pm 0.01$ & $0.47 \pm 0.20$ & $0.47 \pm 0.01$ \\
\hline [O III] $\lambda 4363$ & $0.09 \pm 0.01$ & $0.04 \pm 0.01$ & $0.04 \pm 0.01$ & $0.04 \pm 0.01$ & $0.05 \pm 0.01$ & $0.051 \pm 0.005$ & $0.05 \pm 0.01$ & $0.06 \pm 0.01$ & $\ldots$ & $0.012 \pm 0.002$ \\
\hline Не г $\lambda 4471$ & $\ldots$ & $\ldots$ & $\ldots$ & $\ldots$ & $\ldots$ & $0.036 \pm 0.005$ & $0.03 \pm 0.01$ & $0.038 \pm 0.005$ & $\ldots$ & $0.042 \pm 0.002$ \\
\hline$[\mathrm{Fe}$ III] $\lambda 4658$ & $\ldots$ & $\ldots$ & $\ldots$ & $\ldots$ & $\ldots$ & $\ldots$ & $\ldots$ & $\ldots$ & $\ldots$ & $\ldots$ \\
\hline He II $\lambda 4686$ & $\ldots$ & $\ldots$ & $\ldots$ & $\ldots$ & $\ldots$ & & & $\ldots$ & $\ldots$ & \\
\hline $\mathrm{H} \beta \lambda 4861$ & $1.00 \pm 0.01$ & $1.00 \pm 0.02$ & $1.00 \pm 0.01$ & $1.00 \pm 0.02$ & $1.00 \pm 0.02$ & $1.00 \pm 0.02$ & $1.00 \pm 0.04$ & $1.00 \pm 0.02$ & $1.00 \pm 0.17$ & $1.00 \pm 0.02$ \\
\hline [O III] $\lambda 4959$ & $1.21 \pm 0.02$ & $0.54 \pm 0.01$ & $0.78 \pm 0.01$ & $1.09 \pm 0.02$ & $1.20 \pm 0.02$ & $1.37 \pm 0.03$ & $1.12 \pm 0.02$ & $1.43 \pm 0.03$ & 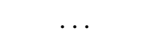 & $0.71 \pm 0.01$ \\
\hline [O III] $\lambda 5007$ & $3.64 \pm 0.07$ & $1.62 \pm 0.03$ & $2.35 \pm 0.05$ & $3.27 \pm 0.07$ & $3.61 \pm 0.07$ & $4.13 \pm 0.08$ & $3.32 \pm 0.07$ & $4.26 \pm 0.09$ & $0.53 \pm 0.15$ & $2.14 \pm 0.04$ \\
\hline [N I] $\lambda 5199$ & $\ldots$ & $\ldots$ & $\ldots$ & $\ldots$ & $\ldots$ & $\ldots$ & $0.013 \pm 0.003$ & $\ldots$ & $\ldots$ & $\ldots$ \\
\hline Не г $\lambda 5876$ & $0.08 \pm 0.01$ & $0.10 \pm 0.01$ & $0.13 \pm 0.01$ & $0.11 \pm 0.01$ & $0.097 \pm 0.004$ & $0.111 \pm 0.003$ & $0.095 \pm 0.005$ & $0.113 \pm 0.004$ & $\ldots$ & $0.12 \pm 0.03$ \\
\hline [O I] $\lambda 6300$ & $0.02 \pm 0.01$ & $0.07 \pm 0.01$ & $0.07 \pm 0.01$ & $0.04 \pm 0.01$ & $0.012 \pm 0.005$ & $0.051 \pm 0.002$ & $0.031 \pm 0.003$ & $0.034 \pm 0.003$ & $\ldots$ & $0.045 \pm 0.002$ \\
\hline [S III] $\lambda 6312$ & $0.03 \pm 0.01$ & 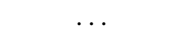 & $\ldots$ & $0.02 \pm 0.01$ & $0.019 \pm 0.005$ & $0.020 \pm 0.002$ & $0.015 \pm 0.003$ & $0.022 \pm 0.003$ & $\ldots$ & $0.016 \pm 0.002$ \\
\hline [O I] $\lambda 6363$ & $0.01 \pm 0.01$ & $0.03 \pm 0.01$ & $0.02 \pm 0.01$ & $0.13 \pm 0.01$ & $\ldots$ & $0.022 \pm 0.002$ & $0.016 \pm 0.003$ & $0.011 \pm 0.003$ & $\ldots$ & $0.013 \pm 0.002$ \\
\hline$[\mathrm{N}$ II] $\lambda 6548$ & $\ldots$ & $0.034 \pm 0.009$ & $0.05 \pm 0.01$ & $0.04 \pm 0.01$ & $0.032 \pm 0.003$ & $0.036 \pm 0.002$ & $0.020 \pm 0.003$ & $0.031 \pm 0.003$ & $\ldots$ & $0.15 \pm 0.01$ \\
\hline $\mathrm{H} \alpha \lambda 6563$ & $2.77 \pm 0.06$ & $2.86 \pm 0.06$ & $2.80 \pm 0.11$ & $2.82 \pm 0.06$ & $2.82 \pm 0.06$ & $2.89 \pm 0.07$ & $2.81 \pm 0.07$ & $2.86 \pm 0.06$ & $2.80 \pm 0.14$ & $2.87 \pm 0.07$ \\
\hline$[\mathrm{N} \mathrm{II}] \lambda 6584$ & $0.05 \pm 0.01$ & $0.125 \pm 0.009$ & $0.14 \pm 0.01$ & $0.12 \pm 0.01$ & $0.122 \pm 0.003$ & $0.109 \pm 0.003$ & $0.080 \pm 0.003$ & $0.092 \pm 0.003$ & $0.14 \pm 0.12$ & $0.48 \pm 0.01$ \\
\hline Не г $\lambda 6678$ & $0.02 \pm 0.01$ & $\ldots$ & $0.04 \pm 0.01$ & $0.029 \pm 0.004$ & $0.028 \pm 0.004$ & $0.027 \pm 0.002$ & $0.025 \pm 0.003$ & $0.034 \pm 0.003$ & $\ldots$ & $0.030 \pm 0.002$ \\
\hline [S II] $\lambda 6717$ & $0.11 \pm 0.01$ & $0.434 \pm 0.009$ & $0.40 \pm 0.02$ & $0.26 \pm 0.01$ & $0.17 \pm 0.01$ & $0.203 \pm 0.004$ & $0.144 \pm 0.004$ & $0.196 \pm 0.004$ & $0.38 \pm 0.10$ & $0.36 \pm 0.01$ \\
\hline$[\mathrm{S}$ II] $\lambda 6731$ & $0.07 \pm 0.01$ & $0.274 \pm 0.009$ & $0.31 \pm 0.02$ & $0.17 \pm 0.01$ & $0.12 \pm 0.01$ & $0.150 \pm 0.003$ & $0.103 \pm 0.003$ & $0.137 \pm 0.003$ & $0.27 \pm 0.10$ & $0.27 \pm 0.01$ \\
\hline$c(\mathrm{H} \beta)$ & $0.00 \pm 0.01$ & $0.17 \pm 0.01$ & $0.23 \pm 0.05$ & $0.28 \pm 0.01$ & $0.22 \pm 0.01$ & $0.43 \pm 0.02$ & $0.41 \pm 0.02$ & $0.29 \pm 0.02$ & $0.63 \pm 0.04$ & $0.43 \pm 0.02$ \\
\hline$F(\mathrm{H} \beta)$ & $8.69 \pm 0.07$ & $13.5 \pm 0.3$ & $9.75 \pm 0.11$ & $20.8 \pm 0.42$ & $19.3 \pm 0.4$ & $22.6 \pm 0.5$ & $29.1 \pm 1.2$ & $24.2 \pm 0.5$ & $0.51 \pm 0.09$ & $117 \pm 2$ \\
\hline $\mathrm{EW}(\mathrm{H} \beta)$ & 30.7 & 21.4 & 19.9 & 25.4 & 129 & 70.1 & 63.7 & 46.6 & 5.9 & 70.1 \\
\hline $\mathrm{EW}(\mathrm{H} \alpha)$ & 130. & 112 & 96.0 & 134 & 622 & 467 & 386 & 269 & 33.3 & 339 \\
\hline
\end{tabular}


Continued)

\begin{tabular}{|c|c|c|c|c|c|c|c|c|c|}
\hline \multirow[b]{2}{*}{ Ion } & \multicolumn{9}{|c|}{$I(\lambda) / I(\mathrm{H} \beta)$} \\
\hline & $\begin{array}{c}\text { NGC } \\
2537 \text { B }\end{array}$ & $\begin{array}{c}\text { UGC } \\
4278 \mathrm{~B}\end{array}$ & $\begin{array}{c}\text { UGC } \\
4278 \mathrm{~A}\end{array}$ & $\begin{array}{c}\text { NGC } \\
2552 \mathrm{~A}\end{array}$ & $\begin{array}{c}\text { UGC } \\
4393 \text { B }\end{array}$ & $\begin{array}{c}\text { UGC } \\
4393 \mathrm{C}\end{array}$ & $\begin{array}{c}\text { CGCG } \\
\text { 035-007 A }\end{array}$ & $\begin{array}{c}\text { UGC } \\
5139 \mathrm{~A}\end{array}$ & $\begin{array}{c}\text { IC } \\
559 \mathrm{~A}\end{array}$ \\
\hline$[\mathrm{O}$ II] $\lambda 3727$ & $2.79 \pm 0.06$ & $2.03 \pm 0.04$ & $1.68 \pm 0.04$ & $2.39 \pm 0.05$ & $2.60 \pm 0.06$ & $4.06 \pm 0.09$ & $4.00 \pm 0.09$ & $1.88 \pm 0.04$ & $3.12 \pm 0.06$ \\
\hline He I $\lambda 3820$ & $\ldots$ & $\ldots$ & $\ldots$ & $\ldots$ & $\ldots$ & $\ldots$ & $\ldots$ & $\ldots$ & $\ldots$ \\
\hline H9 $\lambda 3835$ & $\ldots$ & $0.12 \pm 0.01$ & $0.07 \pm 0.01$ & $\ldots$ & $0.08 \pm 0.01$ & $0.05 \pm 0.01$ & $\ldots$ & $0.05 \pm 0.01$ & \\
\hline$[\mathrm{Ne}$ III] $\lambda 3868$ & $0.11 \pm 0.01$ & $0.17 \pm 0.01$ & $0.20 \pm 0.01$ & $0.25 \pm 0.01$ & $0.27 \pm 0.01$ & $0.26 \pm 0.01$ & $\ldots$ & $0.30 \pm 0.01$ & $0.25 \pm 0.02$ \\
\hline Не I+H8 $\lambda 3889$ & $0.20 \pm 0.01$ & $0.27 \pm 0.01$ & $0.19 \pm 0.01$ & $0.24 \pm 0.01$ & $0.15 \pm 0.01$ & $0.21 \pm 0.01$ & $\ldots$ & $0.19 \pm 0.01$ & $0.16 \pm 0.01$ \\
\hline$[\mathrm{Ne}$ III]+H7 $\lambda 3968$ & $0.15 \pm 0.01$ & $0.43 \pm 0.01$ & $0.23 \pm 0.01$ & $0.50 \pm 0.01$ & $0.39 \pm 0.01$ & $0.24 \pm 0.01$ & $\ldots$ & $0.24 \pm 0.01$ & $0.16 \pm 0.01$ \\
\hline Не г $\lambda 4026$ & $0.024 \pm 0.007$ & $\ldots$ & $\ldots$ & $\ldots$ & $\ldots$ & $0.016 \pm 0.006$ & $\ldots$ & $0.03 \pm 0.01$ & $\ldots$ \\
\hline [S II] $\lambda 4068$ & $0.018 \pm 0.007$ & $0.06 \pm 0.01$ & $0.022 \pm 0.005$ & $0.02 \pm 0.01$ & $\ldots$ & $0.040 \pm 0.005$ & $\ldots$ & $\ldots$ & $\ldots$ \\
\hline $\mathrm{H} \delta \lambda 4101$ & $0.27 \pm 0.01$ & $0.25 \pm 0.01$ & $0.24 \pm 0.01$ & $0.25 \pm 0.01$ & $0.26 \pm 0.01$ & $0.27 \pm 0.01$ & $0.24 \pm 0.02$ & $0.25 \pm 0.01$ & $0.270 \pm 0.008$ \\
\hline $\mathrm{H} \gamma \lambda 4340$ & $0.44 \pm 0.01$ & $0.46 \pm 0.01$ & $0.44 \pm 0.01$ & $0.47 \pm 0.01$ & $0.47 \pm 0.01$ & $0.48 \pm 0.01$ & $0.46 \pm 0.02$ & $0.48 \pm 0.01$ & $0.451 \pm 0.008$ \\
\hline [O III] $\lambda 4363$ & $0.016 \pm 0.004$ & $0.033 \pm 0.005$ & $0.046 \pm 0.004$ & $0.021 \pm 0.003$ & $0.034 \pm 0.008$ & $0.036 \pm 0.005$ & $0.06 \pm 0.02$ & $0.051 \pm 0.008$ & $0.028 \pm 0.008$ \\
\hline Не г $\lambda 4471$ & $0.040 \pm 0.004$ & $0.026 \pm 0.005$ & $0.032 \pm 0.004$ & $0.028 \pm 0.003$ & $0.05 \pm 0.01$ & $0.039 \pm 0.005$ & $\ldots$ & $\ldots$ & $0.025 \pm 0.007$ \\
\hline$[\mathrm{Fe}$ III] $\lambda 4658$ & $\ldots$ & $\ldots$ & $\ldots$ & $\ldots$ & $\ldots$ & $\ldots$ & $\ldots$ & $\ldots$ & $\ldots$ \\
\hline Не II $\lambda 4686$ & $\ldots$ & $\ldots$ & $\ldots$ & $\ldots$ & $\ldots$ & $\ldots$ & $\ldots$ & $\ldots$ & $\ldots$ \\
\hline $\mathrm{H} \beta \lambda 4861$ & $1.00 \pm 0.02$ & $1.00 \pm 0.02$ & $1.00 \pm 0.02$ & $1.00 \pm 0.02$ & $1.00 \pm 0.02$ & $1.00 \pm 0.02$ & $1.00 \pm 0.02$ & $1.00 \pm 0.02$ & $1.00 \pm 0.02$ \\
\hline [O III] $\lambda 4959$ & $0.60 \pm 0.01$ & $0.63 \pm 0.01$ & $0.83 \pm 0.02$ & $0.97 \pm 0.02$ & $1.10 \pm 0.02$ & $0.86 \pm 0.04$ & $0.68 \pm 0.01$ & $1.24 \pm 0.03$ & $0.93 \pm 0.02$ \\
\hline [O III] $\lambda 5007$ & $1.78 \pm 0.04$ & $1.91 \pm 0.04$ & $2.53 \pm 0.05$ & $2.91 \pm 0.06$ & $3.28 \pm 0.07$ & $2.59 \pm 0.05$ & $2.03 \pm 0.04$ & $3.66 \pm 0.07$ & $2.80 \pm 0.06$ \\
\hline$\left[\mathrm{N} \mathrm{I}_{\mathrm{I}}\right] \lambda 5199$ & $\ldots$ & $\ldots$ & $0.007 \pm 0.002$ & $0.013 \pm 0.002$ & $\ldots$ & $\ldots$ & $\ldots$ & $\ldots$ & $\ldots$ \\
\hline He I $\lambda 5876$ & $0.112 \pm 0.002$ & $0.08 \pm 0.01$ & $0.096 \pm 0.004$ & $0.10 \pm 0.01$ & $0.12 \pm 0.01$ & $0.11 \pm 0.01$ & $0.079 \pm 0.013$ & $\ldots$ & $0.09 \pm 0.01$ \\
\hline [O I] $\lambda 6300$ & $0.021 \pm 0.002$ & $0.03 \pm 0.01$ & $0.026 \pm 0.004$ & $0.037 \pm 0.002$ & $0.055 \pm 0.005$ & $0.084 \pm 0.005$ & $\ldots$ & $0.04 \pm 0.01$ & $\ldots$ \\
\hline [S III] $\lambda 6312$ & $0.013 \pm 0.002$ & $0.013 \pm 0.006$ & $0.013 \pm 0.004$ & $0.015 \pm 0.002$ & $0.011 \pm 0.005$ & $0.017 \pm 0.005$ & $\ldots$ & $0.02 \pm 0.01$ & $\ldots$ \\
\hline [O I] $\lambda 6363$ & $0.005 \pm 0.002$ & $0.008 \pm 0.006$ & $0.005 \pm 0.004$ & $0.012 \pm 0.002$ & $0.016 \pm 0.004$ & $0.024 \pm 0.004$ & & $0.06 \pm 0.01$ & \\
\hline$[\mathrm{N} \mathrm{II}] \lambda 6548$ & $0.128 \pm 0.003$ & $0.017 \pm 0.004$ & $0.017 \pm 0.003$ & $0.08 \pm 0.01$ & $0.092 \pm 0.004$ & $0.096 \pm 0.003$ & $0.06 \pm 0.01$ & $0.02 \pm 0.01$ & $0.034 \pm 0.014$ \\
\hline $\mathrm{H} \alpha \lambda 6563$ & $2.79 \pm 0.06$ & $2.80 \pm 0.06$ & $2.86 \pm 0.06$ & $2.86 \pm 0.06$ & $2.86 \pm 0.06$ & $2.87 \pm 0.07$ & $2.84 \pm 0.06$ & $2.83 \pm 0.06$ & $2.86 \pm 0.06$ \\
\hline$\left[\mathrm{N} \mathrm{II}^{\mathrm{II}}\right] \lambda 6584$ & $0.41 \pm 0.01$ & $0.060 \pm 0.004$ & $0.054 \pm 0.003$ & $0.25 \pm 0.01$ & $0.30 \pm 0.01$ & $0.31 \pm 0.01$ & $0.21 \pm 0.01$ & $0.07 \pm 0.01$ & $0.15 \pm 0.01$ \\
\hline Не г $\lambda 6678$ & $0.030 \pm 0.002$ & $0.027 \pm 0.004$ & $0.027 \pm 0.002$ & $0.025 \pm 0.002$ & $0.029 \pm 0.003$ & $0.025 \pm 0.002$ & $\ldots$ & $0.029 \pm 0.006$ & $\ldots$ \\
\hline [S II] $\lambda 6717$ & $0.26 \pm 0.01$ & $0.18 \pm 0.01$ & $0.140 \pm 0.003$ & $0.34 \pm 0.01$ & $0.28 \pm 0.01$ & $0.39 \pm 0.01$ & $0.49 \pm 0.01$ & $0.136 \pm 0.006$ & $0.35 \pm 0.02$ \\
\hline$[\mathrm{S}$ II $] \lambda 6731$ & $0.184 \pm 0.004$ & $0.13 \pm 0.01$ & $0.103 \pm 0.002$ & $0.25 \pm 0.01$ & $0.19 \pm 0.01$ & $0.28 \pm 0.01$ & $0.33 \pm 0.01$ & $0.092 \pm 0.006$ & $0.23 \pm 0.02$ \\
\hline$c(\mathrm{H} \beta)$ & $0.28 \pm 0.01$ & $0.10 \pm 0.01$ & $0.31 \pm 0.02$ & $0.14 \pm 0.01$ & $0.27 \pm 0.02$ & $0.34 \pm 0.02$ & $0.16 \pm 0.01$ & $0.085 \pm 0.010$ & $0.30 \pm 0.02$ \\
\hline$F(\mathrm{H} \beta)$ & $80.0 \pm 1.6$ & $8.93 \pm 0.17$ & $15.9 \pm 0.32$ & $37.0 \pm 0.7$ & $24.2 \pm 0.5$ & $19.3 \pm 0.4$ & $11.3 \pm 0.2$ & $6.40 \pm 0.13$ & $21.3 \pm 0.4$ \\
\hline $\mathrm{EW}(\mathrm{H} \beta)$ & 47.8 & 64.2 & 82.7 & 55.3 & 22.0 & 160 & 16.5 & 197 & 49.7 \\
\hline $\mathrm{EW}(\mathrm{H} \alpha)$ & 188 & 321 & 478 & 312 & 113 & 922 & 72.3 & 939 & 273 \\
\hline
\end{tabular}




\begin{tabular}{|c|c|c|c|c|c|c|c|c|c|}
\hline \multirow[b]{2}{*}{ Ion } & \multicolumn{9}{|c|}{$I(\lambda) / I(\mathrm{H} \beta)$} \\
\hline & $\begin{array}{c}\text { UGC } \\
5272 \mathrm{~A}\end{array}$ & $\begin{array}{c}\text { UGC } \\
5340 \mathrm{~A}\end{array}$ & $\begin{array}{c}\text { UGC } \\
5423 \mathrm{~A}\end{array}$ & $\begin{array}{c}\text { UGC } \\
5423 \text { B }\end{array}$ & $\begin{array}{c}\text { UGC } \\
5672 \mathrm{~A}\end{array}$ & $\begin{array}{c}\text { UGC } \\
5692 \mathrm{~A}\end{array}$ & $\begin{array}{c}\text { UGC } \\
5797 \mathrm{~A}\end{array}$ & $\begin{array}{c}\text { UGC } \\
5923 \mathrm{~A}\end{array}$ & $\begin{array}{c}\text { NGC } \\
3741 \text { A }\end{array}$ \\
\hline [O II] $\lambda 3727$ & $1.06 \pm 0.02$ & $0.58 \pm 0.02$ & $2.08 \pm 0.04$ & $1.78 \pm 0.04$ & $3.28 \pm 0.04$ & $2.47 \pm 0.06$ & $1.59 \pm 0.03$ & $4.06 \pm 0.11$ & $1.60 \pm 0.03$ \\
\hline He I $\lambda 3820$ & $0.014 \pm 0.002$ & & & $\ldots$ & $\ldots$ & $\ldots$ & & & $\ldots$ \\
\hline H9 $\lambda 3835$ & $0.084 \pm 0.002$ & $0.05 \pm 0.01$ & $0.12 \pm 0.01$ & $\ldots$ & $\ldots$ & $\ldots$ & $0.27 \pm 0.04$ & $0.18 \pm 0.03$ & \\
\hline$[\mathrm{Ne}$ III] $\lambda 3868$ & $0.375 \pm 0.007$ & $0.16 \pm 0.01$ & $0.33 \pm 0.01$ & $0.28 \pm 0.02$ & $0.20 \pm 0.04$ & $0.21 \pm 0.05$ & $0.52 \pm 0.04$ & $0.24 \pm 0.03$ & $0.24 \pm 0.01$ \\
\hline Не I+H8 $\lambda 3889$ & $0.197 \pm 0.004$ & $0.17 \pm 0.01$ & $0.24 \pm 0.01$ & $0.32 \pm 0.02$ & $0.16 \pm 0.04$ & $0.24 \pm 0.05$ & $0.36 \pm 0.03$ & $0.33 \pm 0.03$ & $0.21 \pm 0.01$ \\
\hline$[\mathrm{Ne}$ III]+H7 $\lambda 3968$ & $0.194 \pm 0.004$ & $0.47 \pm 0.01$ & $0.28 \pm 0.01$ & $0.50 \pm 0.02$ & $0.19 \pm 0.04$ & $\ldots$ & $0.32 \pm 0.03$ & $0.20 \pm 0.03$ & $0.49 \pm 0.01$ \\
\hline Не г $\lambda 4026$ & $0.015 \pm 0.003$ & $\ldots$ & $\ldots$ & $\ldots$ & $\ldots$ & $\ldots$ & $\ldots$ & $\ldots$ & $\ldots$ \\
\hline [S II] $\lambda 4068$ & & & $0.03 \pm 0.01$ & $\ldots$ & & $\ldots$ & $\ldots$ & $\ldots$ & \\
\hline $\mathrm{H} \delta \lambda 4101$ & $0.26 \pm 0.01$ & $0.25 \pm 0.01$ & $0.26 \pm 0.01$ & $0.26 \pm 0.02$ & $0.25 \pm 0.03$ & $0.22 \pm 0.04$ & $0.28 \pm 0.02$ & $0.24 \pm 0.02$ & $0.25 \pm 0.01$ \\
\hline $\mathrm{H} \gamma \lambda 4340$ & $0.46 \pm 0.01$ & $0.47 \pm 0.01$ & $0.46 \pm 0.01$ & $0.46 \pm 0.01$ & $0.42 \pm 0.03$ & $0.54 \pm 0.03$ & $0.47 \pm 0.02$ & $0.51 \pm 0.02$ & $0.46 \pm 0.01$ \\
\hline [O III] $\lambda 4363$ & $0.083 \pm 0.002$ & $0.06 \pm 0.01$ & $0.070 \pm 0.004$ & $0.07 \pm 0.01$ & $0.04 \pm 0.02$ & $0.06 \pm 0.03$ & $0.09 \pm 0.02$ & $0.06 \pm 0.01$ & $0.06 \pm 0.01$ \\
\hline Не г $\lambda 4471$ & $0.036 \pm 0.002$ & $0.04 \pm 0.01$ & $0.033 \pm 0.004$ & $\ldots$ & $\ldots$ & $\ldots$ & $\ldots$ & $\ldots$ & $0.026 \pm 0.003$ \\
\hline [Fe III] $\lambda 4658$ & $\ldots$ & & & $\ldots$ & $\ldots$ & $\ldots$ & & $\ldots$ & $\ldots$ \\
\hline Не II $\lambda 4686$ & $\cdots$ & $0.024 \pm 0.002$ & $0.019 \pm 0.002$ & $\ldots$ & & $\cdots$ & $0.036 \pm 0.006$ & $\ldots$ & $\cdots$ \\
\hline $\mathrm{H} \beta \lambda 4861$ & $1.00 \pm 0.02$ & $1.00 \pm 0.02$ & $1.00 \pm 0.02$ & $1.00 \pm 0.02$ & $1.00 \pm 0.02$ & $1.00 \pm 0.02$ & $1.00 \pm 0.02$ & $1.00 \pm 0.02$ & $1.00 \pm 0.02$ \\
\hline [O III] $\lambda 4959$ & $1.66 \pm 0.03$ & $0.64 \pm 0.01$ & $1.16 \pm 0.02$ & $1.24 \pm 0.02$ & $0.85 \pm 0.02$ & $0.57 \pm 0.02$ & $1.85 \pm 0.04$ & $0.84 \pm 0.02$ & $0.94 \pm 0.02$ \\
\hline [O III] $\lambda 5007$ & $4.94 \pm 0.10$ & $1.89 \pm 0.04$ & $3.49 \pm 0.01$ & $3.71 \pm 0.07$ & $2.51 \pm 0.05$ & $1.70 \pm 0.03$ & $5.53 \pm 0.11$ & $2.48 \pm 0.05$ & $2.84 \pm 0.06$ \\
\hline$\left[\mathrm{N}_{\mathrm{I}}\right] \lambda 5199$ & $\ldots$ & $\ldots$ & $0.012 \pm 0.004$ & $\ldots$ & $\ldots$ & $\ldots$ & $\ldots$ & $\ldots$ & $\ldots$ \\
\hline Не г $\lambda 5876$ & $0.101 \pm 0.001$ & $0.09 \pm 0.01$ & $0.10 \pm 0.01$ & $0.11 \pm 0.01$ & $0.11 \pm 0.01$ & $0.14 \pm 0.03$ & $0.09 \pm 0.01$ & $\ldots$ & $0.097 \pm 0.003$ \\
\hline [O I] $\lambda 6300$ & $0.013 \pm 0.001$ & $\ldots$ & $0.080 \pm 0.004$ & $0.02 \pm 0.01$ & $0.04 \pm 0.01$ & $\ldots$ & $0.05 \pm 0.02$ & $0.06 \pm 0.01$ & $0.022 \pm 0.002$ \\
\hline [S III] $\lambda 6312$ & $0.020 \pm 0.001$ & $\ldots$ & $0.021 \pm 0.004$ & $0.02 \pm 0.01$ & $\ldots$ & $\ldots$ & $0.02 \pm 0.02$ & $0.02 \pm 0.01$ & $0.018 \pm 0.002$ \\
\hline [O I] $\lambda 6363$ & $0.004 \pm 0.001$ & $\ldots$ & $0.020 \pm 0.004$ & $0.01 \pm 0.01$ & & $\ldots$ & $0.05 \pm 0.02$ & $0.02 \pm 0.01$ & $0.004 \pm 0.002$ \\
\hline$[\mathrm{N}$ II $] \lambda 6548$ & $0.010 \pm 0.001$ & $\ldots$ & $0.035 \pm 0.004$ & $0.026 \pm 0.009$ & $0.12 \pm 0.02$ & $0.10 \pm 0.02$ & $0.03 \pm 0.01$ & $0.07 \pm 0.01$ & $0.017 \pm 0.003$ \\
\hline $\mathrm{H} \alpha \lambda 6563$ & $2.83 \pm 0.06$ & $2.81 \pm 0.06$ & $2.86 \pm 0.06$ & $2.86 \pm 0.06$ & $2.87 \pm 0.07$ & $2.79 \pm 0.06$ & $2.84 \pm 0.06$ & $2.78 \pm 0.07$ & $2.83 \pm 0.06$ \\
\hline$[\mathrm{N} \mathrm{II}] \lambda 6584$ & $0.035 \pm 0.001$ & $0.016 \pm 0.003$ & $0.120 \pm 0.004$ & $0.082 \pm 0.009$ & $0.23 \pm 0.02$ & $0.38 \pm 0.02$ & $0.09 \pm 0.01$ & $0.24 \pm 0.01$ & $0.051 \pm 0.003$ \\
\hline Не г $\lambda 6678$ & $0.029 \pm 0.002$ & $0.023 \pm 0.007$ & $0.027 \pm 0.003$ & $0.029 \pm 0.007$ & & 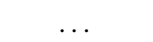 & & $0.03 \pm 0.01$ & $0.026 \pm 0.002$ \\
\hline$\left[\mathrm{S}_{\text {II }}\right] \lambda 6717$ & $0.079 \pm 0.002$ & $0.05 \pm 0.007$ & $0.31 \pm 0.006$ & $0.153 \pm 0.007$ & $0.31 \pm 0.01$ & $0.61 \pm 0.02$ & $0.22 \pm 0.01$ & $0.31 \pm 0.01$ & $0.122 \pm 0.002$ \\
\hline$[\mathrm{S}$ II $] \lambda 6731$ & $0.055 \pm 0.002$ & $0.05 \pm 0.007$ & $0.22 \pm 0.006$ & $0.110 \pm 0.007$ & $0.22 \pm 0.01$ & $0.40 \pm 0.02$ & $0.15 \pm 0.01$ & $0.22 \pm 0.01$ & $0.088 \pm 0.002$ \\
\hline$c(\mathrm{H} \beta)$ & $0.08 \pm 0.01$ & $0.00 \pm 0.01$ & $0.24 \pm 0.01$ & $0.29 \pm 0.02$ & $0.40 \pm 0.02$ & $0.25 \pm 0.01$ & $0.15 \pm 0.01$ & $0.51 \pm 0.02$ & $0.10 \pm 0.01$ \\
\hline$F(\mathrm{H} \beta)$ & $101 \pm 2$ & $13.6 \pm 0.27$ & $22.8 \pm 0.46$ & $9.38 \pm 0.19$ & $5.80 \pm 0.12$ & $4.71 \pm 0.10$ & $8.40 \pm 0.17$ & $20.2 \pm 0.40$ & $45.0 \pm 0.9$ \\
\hline $\mathrm{EW}(\mathrm{H} \beta)$ & 201 & 99.2 & 94.8 & 51.4 & 14.6 & 24.3 & 23.8 & 16.0 & 59.9 \\
\hline $\mathrm{EW}(\mathrm{H} \alpha)$ & 943 & 549 & 438 & 247 & 67.4 & 120 & 111 & 75.3 & 330 \\
\hline
\end{tabular}




\begin{tabular}{|c|c|c|c|c|c|c|c|c|c|}
\hline \multirow{3}{*}{ Ion } & \multicolumn{9}{|c|}{$I(\lambda) / I(\mathrm{H} \beta)$} \\
\hline & $\mathrm{NGC}$ & $\mathrm{NGC}$ & UGC & UGC & NGC & CGCG & CGCG & UGC & NGC \\
\hline & $3738 \mathrm{~A}$ & 3738 B & $6817 \mathrm{~A}$ & $6900 \mathrm{~A}$ & $4163 \mathrm{~A}$ & $269-049 \mathrm{C}$ & 269-049 A & $7577 \mathrm{~A}$ & $4449 \mathrm{C}$ \\
\hline$[\mathrm{O} \mathrm{II]} \lambda 3727$ & $2.91 \pm 0.06$ & $3.45 \pm 0.07$ & $0.94 \pm 0.02$ & $4.13 \pm 0.27$ & $3.72 \pm 0.07$ & $1.76 \pm 0.04$ & $1.02 \pm 0.02$ & $1.65 \pm 0.03$ & $3.26 \pm 0.07$ \\
\hline Не г $\lambda 3820$ & $\ldots$ & $\ldots$ & $\ldots$ & $\ldots$ & $\ldots$ & $\ldots$ & $\ldots$ & $\ldots$ & $\ldots$ \\
\hline H9 $\lambda 3835$ & $\ldots$ & $\ldots$ & $0.08 \pm 0.01$ & $\ldots$ & $\ldots$ & $\ldots$ & $0.09 \pm 0.02$ & $0.06 \pm 0.02$ & $0.04 \pm 0.01$ \\
\hline$[\mathrm{Ne}$ III] $\lambda 3868$ & $0.28 \pm 0.01$ & $0.31 \pm 0.04$ & $0.25 \pm 0.01$ & $\ldots$ & $\ldots$ & $\ldots$ & $0.20 \pm 0.02$ & $0.47 \pm 0.02$ & $0.19 \pm 0.01$ \\
\hline He I+H8 $\lambda 3889$ & $0.11 \pm 0.01$ & $0.32 \pm 0.04$ & $0.19 \pm 0.01$ & $\ldots$ & $\ldots$ & $\ldots$ & $0.16 \pm 0.02$ & $0.20 \pm 0.02$ & $0.17 \pm 0.01$ \\
\hline$[\mathrm{Ne}$ III] $]+\mathrm{H} 7 \lambda 3968$ & $0.39 \pm 0.01$ & $0.24 \pm 0.04$ & $0.53 \pm 0.01$ & $\ldots$ & $\ldots$ & $\ldots$ & $0.44 \pm 0.01$ & $0.34 \pm 0.02$ & $0.19 \pm 0.01$ \\
\hline Не г $\lambda 4026$ & $\ldots$ & $\ldots$ & $\ldots$ & $\ldots$ & $\ldots$ & $\ldots$ & $\ldots$ & $\ldots$ & $\ldots$ \\
\hline$[\mathrm{S} \mathrm{II}] \lambda 4068$ & $\ldots$ & $\ldots$ & $\ldots$ & $\ldots$ & $\ldots$ & $\ldots$ & $\ldots$ & $\ldots$ & $\ldots$ \\
\hline $\mathrm{H} \delta \lambda 4101$ & $0.23 \pm 0.01$ & $0.24 \pm 0.02$ & $0.26 \pm 0.01$ & $0.31 \pm 0.09$ & $0.17 \pm 0.03$ & $0.20 \pm 0.06$ & $0.26 \pm 0.01$ & $0.25 \pm 0.02$ & $0.26 \pm 0.01$ \\
\hline $\mathrm{H} \gamma \lambda 4340$ & $0.47 \pm 0.01$ & $0.45 \pm 0.01$ & $0.46 \pm 0.01$ & $0.55 \pm 0.06$ & $0.31 \pm 0.04$ & $0.29 \pm 0.06$ & $0.46 \pm 0.01$ & $0.47 \pm 0.01$ & $0.45 \pm 0.01$ \\
\hline [O III] $\lambda 4363$ & $0.031 \pm 0.006$ & $0.04 \pm 0.01$ & $0.068 \pm 0.002$ & $\ldots$ & $0.014 \pm 0.003$ & $0.05 \pm 0.01$ & $0.062 \pm 0.003$ & $0.08 \pm 0.01$ & $0.018 \pm 0.004$ \\
\hline Не I $\lambda 4471$ & $0.03 \pm 0.01$ & $\ldots$ & $0.033 \pm 0.002$ & $\ldots$ & $\ldots$ & $\ldots$ & $0.030 \pm 0.003$ & $0.04 \pm 0.01$ & $\ldots$ \\
\hline$[\mathrm{Fe}$ III] $\lambda 4658$ & $\ldots$ & $\ldots$ & $\ldots$ & $\ldots$ & $\ldots$ & $\ldots$ & $\ldots$ & $\ldots$ & $\ldots$ \\
\hline He II $\lambda 4686$ & $\ldots$ & $\ldots$ & $\ldots$ & $\ldots$ & $\ldots$ & $\ldots$ & $\ldots$ & $\ldots$ & $\ldots$ \\
\hline $\mathrm{H} \beta \lambda 4861$ & $1.00 \pm 0.02$ & $1.00 \pm 0.02$ & $1.00 \pm 0.02$ & $1.00 \pm 0.11$ & $1.00 \pm 0.02$ & $1.00 \pm 0.05$ & $1.00 \pm 0.02$ & $1.00 \pm 0.02$ & $1.00 \pm 0.02$ \\
\hline [O III] $\lambda 4959$ & $0.98 \pm 0.02$ & $1.03 \pm 0.02$ & $0.97 \pm 0.02$ & $0.23 \pm 0.10$ & $0.18 \pm 0.02$ & $0.52 \pm 0.04$ & $0.84 \pm 0.02$ & $1.86 \pm 0.04$ & $0.77 \pm 0.02$ \\
\hline [O III] $\lambda 5007$ & $2.96 \pm 0.06$ & $3.11 \pm 0.06$ & $2.89 \pm 0.06$ & $0.60 \pm 0.10$ & $0.49 \pm 0.02$ & $1.53 \pm 0.04$ & $2.51 \pm 0.05$ & $5.25 \pm 0.11$ & $2.33 \pm 0.05$ \\
\hline$\left[\mathrm{NI}_{\mathrm{I}}\right] \lambda 5199$ & $0.011 \pm 0.005$ & & $\ldots$ & $\ldots$ & $\ldots$ & $\ldots$ & $\ldots$ & $\ldots$ & $0.017 \pm 0.003$ \\
\hline He I $\lambda 5876$ & $0.12 \pm 0.01$ & $0.15 \pm 0.02$ & $0.100 \pm 0.002$ & $\ldots$ & $0.10 \pm 0.02$ & $\ldots$ & $0.097 \pm 0.003$ & $0.11 \pm 0.02$ & $0.12 \pm 0.01$ \\
\hline [O I] $\lambda 6300$ & $0.05 \pm 0.01$ & $0.06 \pm 0.01$ & $0.015 \pm 0.002$ & $\ldots$ & $0.07 \pm 0.02$ & $\ldots$ & $0.008 \pm 0.003$ & $\ldots$ & $0.071 \pm 0.004$ \\
\hline [S III] $\lambda 6312$ & $0.02 \pm 0.01$ & $\ldots$ & $0.015 \pm 0.002$ & $\ldots$ & $\ldots$ & $\ldots$ & $0.012 \pm 0.003$ & $\ldots$ & $0.014 \pm 0.004$ \\
\hline [O I] $\lambda 6363$ & $0.02 \pm 0.01$ & $0.02 \pm 0.01$ & $0.004 \pm 0.002$ & $0.29 \pm 0.11$ & $\ldots$ & $\ldots$ & $0.003 \pm 0.001$ & $\ldots$ & $0.024 \pm 0.004$ \\
\hline [N II] $\lambda 6548$ & $0.06 \pm 0.01$ & $0.06 \pm 0.01$ & $0.008 \pm 0.001$ & $0.16 \pm 0.10$ & $0.04 \pm 0.02$ & $0.02 \pm 0.04$ & $0.010 \pm 0.001$ & $0.03 \pm 0.01$ & $0.074 \pm 0.003$ \\
\hline $\mathrm{H} \alpha \lambda 6563$ & $2.83 \pm 0.06$ & $2.82 \pm 0.06$ & $2.83 \pm 0.06$ & $2.83 \pm 0.10$ & $2.75 \pm 0.06$ & $2.79 \pm 0.06$ & $2.83 \pm 0.06$ & $2.79 \pm 0.06$ & $2.84 \pm 0.06$ \\
\hline [N II] $\lambda 6584$ & $0.19 \pm 0.01$ & $0.20 \pm 0.01$ & $0.03 \pm 0.01$ & $0.52 \pm 0.10$ & $0.13 \pm 0.02$ & $\ldots$ & $0.033 \pm 0.003$ & $0.09 \pm 0.01$ & $0.23 \pm 0.01$ \\
\hline Не г $\lambda 6678$ & $0.03 \pm 0.01$ & $\ldots$ & $0.026 \pm 0.002$ & $\ldots$ & $\ldots$ & $\ldots$ & $0.024 \pm 0.002$ & $0.03 \pm 0.01$ & $0.020 \pm 0.003$ \\
\hline$[\mathrm{S}$ II] $\lambda 6717$ & $0.32 \pm 0.01$ & $0.29 \pm 0.01$ & $0.068 \pm 0.002$ & $1.11 \pm 0.09$ & $0.35 \pm 0.02$ & $\ldots$ & $0.064 \pm 0.002$ & $0.20 \pm 0.01$ & $0.35 \pm 0.01$ \\
\hline$[\mathrm{S}$ II] $\lambda 6731$ & $0.23 \pm 0.01$ & $0.21 \pm 0.01$ & $0.049 \pm 0.002$ & $0.64 \pm 0.09$ & $0.23 \pm 0.02$ & $\ldots$ & $0.046 \pm 0.002$ & $0.15 \pm 0.01$ & $0.25 \pm 0.01$ \\
\hline$c(\mathrm{H} \beta)$ & $0.04 \pm 0.01$ & $0.19 \pm 0.01$ & $0.06 \pm 0.01$ & $0.09 \pm 0.01$ & $0.10 \pm 0.01$ & $0.16 \pm 0.01$ & $0.08 \pm 0.01$ & $0.05 \pm 0.01$ & $0.14 \pm 0.01$ \\
\hline$F(\mathrm{H} \beta)$ & $58.7 \pm 1.2$ & $24.8 \pm 0.5$ & $42.3 \pm 0.8$ & $1.49 \pm 0.17$ & $5.24 \pm 0.12$ & $1.71 \pm 0.08$ & $29.1 \pm 0.6$ & $12.5 \pm 0.3$ & $54.8 \pm 1.1$ \\
\hline $\mathrm{EW}(\mathrm{H} \beta)$ & 35.2 & 23.4 & 146 & 20.0 & 9.21 & 6.8 & 81.3 & 216 & 119 \\
\hline $\mathrm{EW}(\mathrm{H} \alpha)$ & 183 & 121 & 834 & 75.5 & 40.9 & 37.2 & 434 & 854 & 437 \\
\hline
\end{tabular}




\begin{tabular}{|c|c|c|c|c|c|c|c|c|c|}
\hline \multirow[b]{2}{*}{ Ion } & \multicolumn{9}{|c|}{$I(\lambda) / I(\mathrm{H} \beta)$} \\
\hline & $\begin{array}{c}\text { NGC } \\
4449 \text { B }\end{array}$ & $\begin{array}{c}\text { NGC } \\
4449 \text { A }\end{array}$ & $\begin{array}{c}\text { UGC } \\
7605 \mathrm{~A}\end{array}$ & $\begin{array}{c}\text { UGC } \\
7639 \mathrm{~A}\end{array}$ & $\begin{array}{c}\text { NGC } \\
4656 \mathrm{~A}\end{array}$ & $\begin{array}{c}\text { UGC } \\
8201 \mathrm{~A}\end{array}$ & $\begin{array}{c}\text { UGC } \\
8245 \mathrm{~A}\end{array}$ & $\begin{array}{c}\text { UGC } \\
8508 \mathrm{~A}\end{array}$ & $\begin{array}{c}\text { UGC } \\
8638 \mathrm{~A}\end{array}$ \\
\hline [O II] $\lambda 3727$ & $3.04 \pm 0.07$ & $2.39 \pm 0.05$ & $1.91 \pm 0.04$ & $3.98 \pm 0.12$ & $0.80 \pm 0.03$ & $1.62 \pm 0.03$ & $2.81 \pm 0.06$ & $1.47 \pm 0.03$ & $1.77 \pm 0.04$ \\
\hline Не г $\lambda 3820$ & $\ldots$ & $0.008 \pm 0.004$ & $\ldots$ & $\ldots$ & $\ldots$ & $\ldots$ & $\ldots$ & $\ldots$ & $\ldots$ \\
\hline H9 $\lambda 3835$ & $0.05 \pm 0.01$ & $0.062 \pm 0.004$ & $\ldots$ & $\ldots$ & $0.08 \pm 0.02$ & $\ldots$ & $\ldots$ & $\ldots$ & $\ldots$ \\
\hline$[\mathrm{Ne}$ III] $\lambda 3868$ & $0.19 \pm 0.01$ & $0.23 \pm 0.01$ & $0.18 \pm 0.02$ & $\ldots$ & $0.50 \pm 0.02$ & $0.25 \pm 0.01$ & $\ldots$ & $0.29 \pm 0.02$ & $0.32 \pm 0.01$ \\
\hline $\mathrm{He} \mathrm{I}+\mathrm{H} 8 \lambda 3889$ & $0.19 \pm 0.01$ & $0.167 \pm 0.004$ & $0.15 \pm 0.02$ & $\ldots$ & $0.19 \pm 0.02$ & $0.23 \pm 0.01$ & $\ldots$ & $0.26 \pm 0.02$ & $0.22 \pm 0.01$ \\
\hline$[\mathrm{Ne}$ III] $]+\mathrm{H} 7 \lambda 3968$ & $0.19 \pm 0.01$ & $0.187 \pm 0.004$ & $0.18 \pm 0.02$ & $\ldots$ & $0.29 \pm 0.02$ & $0.19 \pm 0.01$ & $\ldots$ & $0.49 \pm 0.02$ & $0.59 \pm 0.01$ \\
\hline Не г $\lambda 4026$ & $\ldots$ & $0.016 \pm 0.001$ & $\ldots$ & $\ldots$ & $\ldots$ & $\ldots$ & $\ldots$ & $\ldots$ & $\ldots$ \\
\hline$[\mathrm{S} \mathrm{II}] \lambda 4068$ & $\ldots$ & $0.014 \pm 0.001$ & $\ldots$ & $\ldots$ & $\ldots$ & $\ldots$ & $\ldots$ & $\ldots$ & $\ldots$ \\
\hline $\mathrm{H} \delta \lambda 4101$ & $0.25 \pm 0.01$ & $0.26 \pm 0.01$ & $0.25 \pm 0.02$ & $0.28 \pm 0.05$ & $0.25 \pm 0.02$ & $0.26 \pm 0.01$ & $0.24 \pm 0.02$ & $0.24 \pm 0.02$ & $0.26 \pm 0.01$ \\
\hline $\mathrm{H} \gamma \lambda 4340$ & $0.48 \pm 0.01$ & $0.47 \pm 0.01$ & $0.46 \pm 0.01$ & $0.44 \pm 0.07$ & $0.45 \pm 0.01$ & $0.47 \pm 0.01$ & $0.41 \pm 0.02$ & $0.45 \pm 0.01$ & $0.45 \pm 0.01$ \\
\hline [O III] $\lambda 4363$ & $0.018 \pm 0.003$ & $0.019 \pm 0.001$ & $0.04 \pm 0.01$ & $0.05 \pm 0.05$ & $0.09 \pm 0.01$ & $0.05 \pm 0.01$ & $0.03 \pm 0.02$ & $0.06 \pm 0.01$ & $0.055 \pm 0.003$ \\
\hline Не г $\lambda 4471$ & $0.032 \pm 0.003$ & $0.039 \pm 0.001$ & $0.02 \pm 0.01$ & $\ldots$ & $\ldots$ & $0.05 \pm 0.01$ & $\ldots$ & $0.03 \pm 0.01$ & $0.039 \pm 0.002$ \\
\hline$[\mathrm{Fe}$ III] $\lambda 4658$ & $\ldots$ & $\ldots$ & $\ldots$ & $\ldots$ & $\ldots$ & $\ldots$ & $\ldots$ & $\ldots$ & $\ldots$ \\
\hline He II $\lambda 4686$ & $\ldots$ & $\ldots$ & $\ldots$ & $\ldots$ & $\ldots$ & $\ldots$ & $0.05 \pm 0.01$ & $\ldots$ & $\ldots$ \\
\hline $\mathrm{H} \beta \lambda 4861$ & $1.00 \pm 0.02$ & $1.00 \pm 0.02$ & $1.00 \pm 0.02$ & $1.00 \pm 0.05$ & $1.00 \pm 0.02$ & $1.00 \pm 0.02$ & $1.00 \pm 0.02$ & $1.00 \pm 0.02$ & $1.00 \pm 0.02$ \\
\hline [O III] $\lambda 4959$ & $0.84 \pm 0.02$ & $1.15 \pm 0.02$ & $0.76 \pm 0.01$ & $0.48 \pm 0.06$ & $2.29 \pm 0.05$ & $0.99 \pm 0.06$ & $0.43 \pm 0.01$ & $1.08 \pm 0.02$ & $1.39 \pm 0.03$ \\
\hline [O III] $\lambda 5007$ & $2.54 \pm 0.05$ & $3.46 \pm 0.07$ & $2.33 \pm 0.05$ & $1.33 \pm 0.06$ & $6.71 \pm 0.13$ & $2.94 \pm 0.06$ & $1.25 \pm 0.03$ & $3.25 \pm 0.06$ & $4.17 \pm 0.08$ \\
\hline [NI] $\lambda 5199$ & $0.013 \pm 0.002$ & $0.006 \pm 0.001$ & $\ldots$ & $\ldots$ & $\ldots$ & $\ldots$ & $\ldots$ & $\ldots$ & $\ldots$ \\
\hline He I $\lambda 5876$ & $0.106 \pm 0.002$ & $0.115 \pm 0.002$ & $0.07 \pm 0.02$ & $\ldots$ & $\ldots$ & $0.11 \pm 0.01$ & $0.10 \pm 0.02$ & $0.10 \pm 0.01$ & $0.112 \pm 0.002$ \\
\hline [O I] $\lambda 6300$ & $0.050 \pm 0.002$ & $0.028 \pm 0.001$ & $0.05 \pm 0.02$ & $0.09 \pm 0.06$ & $0.02 \pm 0.01$ & $0.06 \pm 0.01$ & $0.15 \pm 0.02$ & $0.04 \pm 0.01$ & $0.016 \pm 0.001$ \\
\hline [S III] $\lambda 6312$ & $0.012 \pm 0.002$ & $0.014 \pm 0.001$ & $0.01 \pm 0.02$ & $\ldots$ & $0.03 \pm 0.01$ & $0.02 \pm 0.01$ & $\ldots$ & $0.02 \pm 0.01$ & $0.021 \pm 0.001$ \\
\hline [O I] $\lambda 6363$ & $0.015 \pm 0.002$ & $0.009 \pm 0.001$ & $\ldots$ & $\ldots$ & $0.01 \pm 0.01$ & $0.04 \pm 0.01$ & $0.08 \pm 0.02$ & $0.02 \pm 0.01$ & $0.008 \pm 0.001$ \\
\hline [N II] $\lambda 6548$ & $0.074 \pm 0.002$ & $0.048 \pm 0.001$ & $0.015 \pm 0.018$ & $0.07 \pm 0.06$ & $0.009 \pm 0.009$ & $\ldots$ & $0.04 \pm 0.02$ & $\ldots$ & $0.02 \pm 0.01$ \\
\hline $\mathrm{H} \alpha \lambda 6563$ & $2.86 \pm 0.06$ & $2.83 \pm 0.06$ & $2.83 \pm 0.06$ & $2.83 \pm 0.06$ & $2.86 \pm 0.06$ & $2.81 \pm 0.06$ & $2.83 \pm 0.06$ & $2.83 \pm 0.06$ & $2.82 \pm 0.06$ \\
\hline [N II] $\lambda 6584$ & $0.205 \pm 0.004$ & $0.163 \pm 0.003$ & $0.066 \pm 0.018$ & $0.21 \pm 0.06$ & $0.023 \pm 0.009$ & $0.04 \pm 0.01$ & $0.12 \pm 0.01$ & $0.05 \pm 0.01$ & $0.07 \pm 0.01$ \\
\hline Не г $\lambda 6678$ & $0.025 \pm 0.002$ & $0.029 \pm 0.001$ & $0.05 \pm 0.01$ & $\ldots$ & $\ldots$ & $0.03 \pm 0.01$ & $0.02 \pm 0.01$ & $0.029 \pm 0.001$ & $0.028 \pm 0.002$ \\
\hline$[\mathrm{S}$ II] $\lambda 6717$ & $0.28 \pm 0.01$ & $0.18 \pm 0.01$ & $0.15 \pm 0.01$ & $0.45 \pm 0.04$ & $0.07 \pm 0.01$ & $0.10 \pm 0.01$ & $0.27 \pm 0.01$ & $0.12 \pm 0.01$ & $0.14 \pm 0.01$ \\
\hline$[\mathrm{S}$ II] $\lambda 6731$ & $0.20 \pm 0.01$ & $0.13 \pm 0.01$ & $0.09 \pm 0.01$ & $0.28 \pm 0.04$ & $0.04 \pm 0.01$ & $0.07 \pm 0.01$ & $0.18 \pm 0.01$ & $0.08 \pm 0.01$ & $0.10 \pm 0.01$ \\
\hline$c(\mathrm{H} \beta)$ & $0.21 \pm 0.01$ & $0.09 \pm 0.01$ & $0.06 \pm 0.01$ & $0.08 \pm 0.01$ & $0.24 \pm 0.01$ & $0.15 \pm 0.01$ & $0.11 \pm 0.01$ & $0.09 \pm 0.01$ & $0.002 \pm 0.001$ \\
\hline$F(\mathrm{H} \beta)$ & $64.4 \pm 1.3$ & $567 \pm 11$ & $6.37 \pm 0.13$ & $1.70 \pm 0.09$ & $43.4 \pm 0.9$ & $8.87 \pm 0.18$ & $6.43 \pm 0.13$ & $9.79 \pm 0.20$ & $41.4 \pm 0.8$ \\
\hline $\mathrm{EW}(\mathrm{H} \beta)$ & 119 & 239 & 75.8 & 8.25 & 171 & 120 & 20.8 & 74.0 & 129 \\
\hline $\mathrm{EW}(\mathrm{H} \alpha)$ & 587 & 851 & 611 & 37.8 & 1141 & 547 & 91.1 & 314 & 601 \\
\hline
\end{tabular}


Table 3

(Continued)

\begin{tabular}{|c|c|c|c|c|c|c|}
\hline \multirow{3}{*}{ Ion } & \multicolumn{6}{|c|}{$I(\lambda) / I(\mathrm{H} \beta)$} \\
\hline & UGC & UGC & NGC & UGC & UGC & $\mathrm{KKH}$ \\
\hline & 8638 B & $8837 \mathrm{~A}$ & $5477 \mathrm{~A}$ & $9405 \mathrm{~A}$ & $10818 \mathrm{~A}$ & $098 \mathrm{~A}$ \\
\hline [O II] $\lambda 3727$ & $1.75 \pm 0.04$ & $3.46 \pm 0.01$ & $1.31 \pm 0.05$ & $3.66 \pm 0.37$ & $2.73 \pm 0.06$ & $1.85 \pm 0.05$ \\
\hline He г $\lambda 3820$ & $\ldots$ & $\ldots$ & $\ldots$ & $\ldots$ & $\ldots$ & $\ldots$ \\
\hline H9 $\lambda 3835$ & $0.10 \pm 0.01$ & $0.054 \pm 0.005$ & $0.06 \pm 0.01$ & $\ldots$ & $0.16 \pm 0.02$ & $\ldots$ \\
\hline [Ne III] $\lambda 3868$ & $0.32 \pm 0.01$ & $0.071 \pm 0.005$ & $0.33 \pm 0.01$ & $\ldots$ & $0.20 \pm 0.02$ & $0.21 \pm 0.04$ \\
\hline He I+H8 $\lambda 3889$ & $0.24 \pm 0.01$ & $0.21 \pm 0.01$ & $0.23 \pm 0.01$ & $\ldots$ & $0.19 \pm 0.02$ & $0.17 \pm 0.04$ \\
\hline$[\mathrm{Ne}$ III]+H7 $\lambda 3968$ & $0.02 \pm 0.01$ & $0.16 \pm 0.01$ & $0.33 \pm 0.01$ & $\ldots$ & $0.28 \pm 0.01$ & $0.09 \pm 0.04$ \\
\hline Не г $\lambda 4026$ & $\ldots$ & $\ldots$ & $\ldots$ & $\ldots$ & $\ldots$ & $\ldots$ \\
\hline [S II] $\lambda 4068$ & $0.02 \pm 0.01$ & $0.02 \pm 0.01$ & $\ldots$ & $\ldots$ & 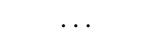 & $\ldots$ \\
\hline $\mathrm{H} \delta \lambda 4101$ & $0.27 \pm 0.01$ & $0.24 \pm 0.01$ & $0.28 \pm 0.02$ & $\ldots$ & $0.23 \pm 0.01$ & $0.36 \pm 0.03$ \\
\hline $\mathrm{H} \gamma \lambda 4340$ & $0.47 \pm 0.01$ & $0.47 \pm 0.01$ & $0.47 \pm 0.02$ & $0.35 \pm 0.22$ & $0.45 \pm 0.01$ & $0.58 \pm 0.02$ \\
\hline [O III] $\lambda 4363$ & $0.06 \pm 0.01$ & $0.019 \pm 0.003$ & $0.06 \pm 0.01$ & $\ldots$ & $0.02 \pm 0.01$ & $\ldots$ \\
\hline Не г $\lambda 4471$ & $0.03 \pm 0.01$ & $0.030 \pm 0.003$ & $\ldots$ & $\ldots$ & $0.03 \pm 0.01$ & $\ldots$ \\
\hline$[\mathrm{Fe}$ III] $\lambda 4658$ & $\ldots$ & $0.009 \pm 0.003$ & $\ldots$ & $\ldots$ & $\ldots$ & $\ldots$ \\
\hline Не II $\lambda 4686$ & $\ldots$ & $\ldots$ & $\ldots$ & $\ldots$ & $\ldots$ & $\ldots$ \\
\hline $\mathrm{H} \beta \lambda 4861$ & $1.00 \pm 0.02$ & $1.00 \pm 0.02$ & $1.00 \pm 0.02$ & $1.00 \pm 0.13$ & $1.00 \pm 0.02$ & $1.00 \pm 0.02$ \\
\hline [O III] $\lambda 4959$ & $1.39 \pm 0.03$ & $0.42 \pm 0.01$ & $1.55 \pm 0.03$ & $0.46 \pm 0.14$ & $0.74 \pm 0.02$ & $0.63 \pm 0.01$ \\
\hline [O III] $\lambda 5007$ & $4.15 \pm 0.08$ & $1.26 \pm 0.03$ & $4.64 \pm 0.10$ & $1.44 \pm 0.14$ & $2.22 \pm 0.04$ & $1.91 \pm 0.01$ \\
\hline [N I] $\lambda 5199$ & $\ldots$ & $0.012 \pm 0.002$ & $\ldots$ & $\ldots$ & $0.02 \pm 0.01$ & $\ldots$ \\
\hline Не г $\lambda 5876$ & $0.107 \pm 0.004$ & $0.091 \pm 0.002$ & $0.11 \pm 0.01$ & $\ldots$ & $0.10 \pm 0.01$ & $0.08 \pm 0.02$ \\
\hline [O I] $\lambda 6300$ & $0.013 \pm 0.003$ & $0.051 \pm 0.002$ & $0.01 \pm 0.01$ & $\ldots$ & $0.06 \pm 0.01$ & $\ldots$ \\
\hline [S III] $\lambda 6312$ & $0.018 \pm 0.003$ & $0.018 \pm 0.002$ & $0.02 \pm 0.01$ & $\ldots$ & $0.01 \pm 0.01$ & $\ldots$ \\
\hline [O I] $\lambda 6363$ & $0.004 \pm 0.003$ & $0.013 \pm 0.002$ & $0.05 \pm 0.01$ & $\ldots$ & $0.02 \pm 0.01$ & $\ldots$ \\
\hline$[\mathrm{N}$ II $] \lambda 6548$ & $0.02 \pm 0.01$ & $0.067 \pm 0.002$ & $0.015 \pm 0.004$ & $\ldots$ & $0.08 \pm 0.01$ & $0.04 \pm 0.02$ \\
\hline $\mathrm{H} \alpha \lambda 6563$ & $2.81 \pm 0.06$ & $2.83 \pm 0.06$ & $2.84 \pm 0.11$ & $2.79 \pm 0.17$ & $2.79 \pm 0.06$ & $2.79 \pm 0.06$ \\
\hline$[\mathrm{N}$ II $] \lambda 6584$ & $0.06 \pm 0.01$ & $0.202 \pm 0.004$ & $0.047 \pm 0.001$ & $0.40 \pm 0.16$ & $0.25 \pm 0.01$ & $0.08 \pm 0.02$ \\
\hline Не г $\lambda 6678$ & $0.026 \pm 0.003$ & $0.021 \pm 0.002$ & $0.028 \pm 0.001$ & $\ldots$ & $\ldots$ & $\ldots$ \\
\hline$[\mathrm{S}$ II] $\lambda 6717$ & $0.11 \pm 0.01$ & $0.35 \pm 0.01$ & $0.079 \pm 0.002$ & $0.76 \pm 0.15$ & $\ldots$ & $0.15 \pm 0.02$ \\
\hline$[\mathrm{S}$ II $] \lambda 6731$ & $0.09 \pm 0.01$ & $0.25 \pm 0.01$ & $0.059 \pm 0.001$ & $0.55 \pm 0.14$ & $\ldots$ & $0.10 \pm 0.02$ \\
\hline$c(\mathrm{H} \beta)$ & $0.24 \pm 0.01$ & $0.06 \pm 0.01$ & $0.09 \pm 0.01$ & $0.10 \pm 0.01$ & $0.19 \pm 0.01$ & $0.24 \pm 0.01$ \\
\hline$F(\mathrm{H} \beta)$ & $16.7 \pm 0.3$ & $35.4 \pm 0.71$ & $78.0 \pm 1.6$ & $0.48 \pm 0.06$ & $11.3 \pm 0.2$ & $3.07 \pm 0.06$ \\
\hline $\mathrm{EW}(\mathrm{H} \beta)$ & 57.1 & 114 & 177 & 43.6 & 46.3 & 50.6 \\
\hline $\mathrm{EW}(\mathrm{H} \alpha)$ & 301 & 723 & 879 & 216 & 216 & 224 \\
\hline
\end{tabular}

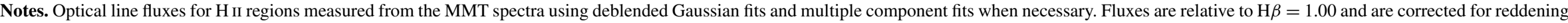

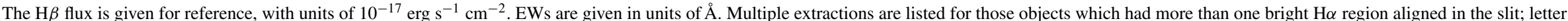

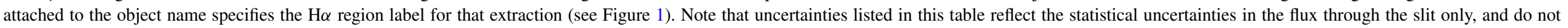
account for slit losses. 
calculated using the York Extinction Solver (McCall 2004). ${ }^{15}$ With these values, the reddening, $E(B-V)$, can be derived using

$$
\log \frac{I(\mathrm{H} \alpha)}{I(\mathrm{H} \beta)}=\log \frac{F(\mathrm{H} \alpha)}{F(\mathrm{H} \beta)}+0.4 E(B-V)\left[A_{1}(\mathrm{H} \alpha)-A_{1}(\mathrm{H} \beta)\right],
$$

where $F(\mathrm{H} \alpha) / F(\mathrm{H} \beta)$ is the observed flux ratio and $I(\mathrm{H} \alpha) / I(\mathrm{H} \beta)$ is the de-reddened line intensity ratio using case $\mathrm{B}$ from Hummer \& Storey (1987), assuming an electron temperature calculated from the [O III] line ratio and $n_{\mathrm{e}}=10^{2} \mathrm{~cm}^{-3}$. For our sample, the electron temperature range is $9500 \mathrm{~K}-19,500 \mathrm{~K}$, with an average of $13,300 \mathrm{~K}$. This range agrees with the typical electron temperatures of $10,000 \mathrm{~K}-20,000 \mathrm{~K}$ for metal-poor $\mathrm{H}$ II regions. This same process can be carried out for the $\mathrm{H} \gamma / \mathrm{H} \beta$ and $\mathrm{H} \delta / \mathrm{H} \beta$ ratios observed. When all the necessary Balmer lines were present, which is true of all of the objects in our "Select" sample, we used a minimized chi-squared approach to find the best estimate of $E(B-V)$ based on the $\mathrm{H} \alpha / \mathrm{H} \beta, \mathrm{H} \gamma / \mathrm{H} \beta$, and $\mathrm{H} \delta / \mathrm{H} \beta$ ratios. The resulting Balmer ratios are within errors of the Hummer \& Storey (1987) Case B values for all objects meeting the selection criteria of our "Select" sample (see Section 6.1), with an average of $\chi^{2}=0.03$.

Following Lee \& Skillman (2004), the reddening value can be converted to the logarithmic extinction at $\mathrm{H} \beta$ as

$$
c(\mathrm{H} \beta)=1.43 E(B-V) .
$$

Our reddening corrections are tabulated in Table 3.

\section{5. "DIRECT" OXYGEN ABUNDANCE DETERMINATIONS}

Accurate "direct" oxygen abundance determinations from $\mathrm{H}$ II regions require a measurement of the electron temperature (typically via observation of the temperature sensitive auroral [O III] $\lambda 4363$ line). For the 31 low-luminosity objects for which [O III] $\lambda 4363$ strengths were measured to be $>4 \sigma$, we use the temperature sensitive ratio comparing "auroral" to "nebular" collisionally excited lines to determine electron temperatures. A simple, yet reasonable, approximation to the geometry of an $\mathrm{H}$ II region is to assume a two-zone volume, where $t_{2}$ and $t_{3}$ are the electron temperatures (in units of $10^{4} \mathrm{~K}$ ) in the low and high ionization zones, respectively. For the high ionization zone, the $[\mathrm{O}$ III $] I(\lambda \lambda 4959,5007) / I(\lambda 4363)$ ratio was used to derive a temperature using the IRAF task TEMDEN. This task computes the electron temperature of the ionized nebular gas within the 5level atom approximation. The $\mathrm{O}^{+}$(low ionization) zone electron temperature can be related to the $\mathrm{O}^{++}$(high ionization) zone electron temperature (e.g., Campbell et al. 1986; Pagel et al. 1992). We used the relation between $t_{2}$ and $t_{3}$ proposed by Pagel et al. (1992), based on the photoionization modeling of Stasińska (1990) to determine the low ionization zone temperature:

$$
t_{2}^{-1}=0.5\left(t_{3}^{-1}+0.8\right) \text {. }
$$

The low and high ionization region temperatures are tabulated in Table 4. Typically $\mathrm{H}$ II regions are assumed to have electron temperatures within the range of 1 to $2 \times 10^{4} \mathrm{~K}$. Temperatures for the present sample agree with this approximation, spanning $10,800 \mathrm{~K}-15,200 \mathrm{~K}$ for the low ionization region, and $9600 \mathrm{~K}-19,400 \mathrm{~K}$ for the high ionization region.

\footnotetext{
15 http://www1.cadc-ccda.hia-iha.nrc-cnrc.gc.ca/ community/YorkExtinctionSolver/
}

Since the MMT spectra include emission lines from both $\mathrm{O}^{+}$ and $\mathrm{O}^{++}$, we determine oxygen abundances based on our estimated two-zone electron temperatures. Spectra which contained measurable $\left[\mathrm{S}_{\mathrm{II}}\right] \lambda \lambda 6717,6731$ were used to determine electron densities consistent with the low density limit. Thus, it is reasonable to simply assume $n_{\mathrm{e}}=10^{2} \mathrm{~cm}^{-3}$ for this sample. Ionic abundances were calculated with

$$
\frac{N\left(\mathrm{X}^{i}\right)}{N\left(\mathrm{H}^{+}\right)}=\frac{I_{\lambda(i)}}{I_{\mathrm{H} \beta}} \frac{j_{\mathrm{H} \beta}}{j_{\lambda(i)}} .
$$

The emissivity coefficients, which are functions of both temperature and density, were determined using the IONIC routine in IRAF. This routine applies the 5-level atom approximation, assuming the appropriate ionization zone electron temperature, as determined from the oxygen line ratios.

Some abundance determinations require ionization correction factors to account for unobserved ionic species. Here, we assume $\mathrm{N} / \mathrm{O}=\mathrm{N}^{+} / \mathrm{O}^{+}$(Peimbert \& Costero 1969). Nava et al. (2006) have investigated the validity of this assumption. They concluded that although it could be improved upon with modern photoionization models, it is valid to within about $10 \%$. Thus, we employ this assumption, mostly for the purposes of direct comparison with other studies in the literature.

For the nine objects with multiple $\mathrm{H}$ II regions containing strong [O III] $\lambda 4363$, an error weighted average was used to determine a best estimate of relative abundances and oxygen abundances. The results from individual $\mathrm{H}$ II regions are tabulated in Table 4 and the mean values, using a weight of $1 / \sigma_{i}^{2}$ for each component, are listed in Table 5. The uncertainties for these mean values are represented by the standard deviation of the weighted mean or the weighted dispersion, whichever is greater. Calculated errors in this paper provide a statistical estimate only. Additional errors may be important, such as systematic errors due to temperature fluctuations or other imperfect assumptions. However, the purpose of this paper is to improve the $L-Z$ and $M-Z$ relationships with abundances from high quality spectra. The statistical errors allow such an assessment of the relative quality of the spectra used, which in turn are weighted higher in the regression fits.

For seven of the nine dwarf galaxies with direct abundances from multiple $\mathrm{H}$ II regions, the derived oxygen abundances agree within the uncertainties. These support the interpretation that the interstellar medium (ISM) in typical dwarf galaxies is chemically well mixed, in agreement with past studies (e.g., Skillman et al. 1989; Kobulnicky \& Skillman 1996, 1997; Lee et al. 2006b; Kehrig et al. 2008; Croxall et al. 2009; Pérez-Montero et al. 2011). Various theoretical studies support this result (e.g., Roy \& Kunth 1995). However, there are two galaxies for which the oxygen abundances do not agree. For NGC 4449 the highest signal-to-noise spectrum is offset to higher $\log (\mathrm{O} / \mathrm{H})$ values by 0.16 and 0.18 dex compared to the other two. This discrepancy may be due to the possible contamination of an embedded supernova remnant (e.g., Skillman 1985) or it may be truly offset. Additional spectra are needed to clarify this. NGC 2537 has two high quality optical spectra, but the derived values disagree by 0.26 dex. This difference by a factor of nearly two is intriguing, warranting further investigation of this object. We increased the error of the weighted mean to indicate the dispersion between the two values. Note that the lower value would be in better agreement with the $L-Z$ relationships, but that the mean is not offset very far. Overall, the oxygen abundances determined in this paper are all relatively low $(12+$ $\log (\mathrm{O} / \mathrm{H})<8.3$; average $12+\log (\mathrm{O} / \mathrm{H})=7.84)$ as we would 
Table 4

Ionic and Total Abundances

\begin{tabular}{|c|c|c|c|c|c|c|c|c|c|c|}
\hline Galaxy & $\mathrm{H} \alpha$ Region & $\begin{array}{c}t_{2} \\
(\mathrm{~K})\end{array}$ & $\begin{array}{c}t_{3} \\
(\mathrm{~K})\end{array}$ & $\begin{array}{l}\mathrm{O}^{+} / \mathrm{H}^{+} \\
\left(\times 10^{5}\right)\end{array}$ & $\begin{array}{l}\mathrm{O}^{++} / \mathrm{H}^{+} \\
\left(\times 10^{5}\right)\end{array}$ & $\begin{array}{c}\mathrm{O} / \mathrm{H} \\
\left(\times 10^{5}\right)\end{array}$ & $\begin{array}{c}12+\log (\mathrm{O} / \mathrm{H}) \\
(\operatorname{dex})\end{array}$ & $\begin{array}{l}\mathrm{N}^{+} / \mathrm{H}^{+} \\
\left(\times 10^{6}\right)\end{array}$ & $\begin{array}{l}\log (\mathrm{N} / \mathrm{O}) \\
\quad(\mathrm{dex})\end{array}$ & $\begin{array}{c}\mathrm{N} / \mathrm{H} \\
\left(\times 10^{6}\right)\end{array}$ \\
\hline UGC 521 & A & $14200 \pm 900$ & $16500 \pm 1100$ & $1.59 \pm 0.32$ & $3.05 \pm 0.42$ & $4.64 \pm 0.53$ & $7.67 \pm 0.05$ & $0.29 \pm 0.06$ & $-1.61 \pm 0.07$ & $1.15 \pm 0.25$ \\
\hline UGC 695 & $\mathrm{E}$ & $14000 \pm 1900$ & $15800 \pm 2200$ & $3.45 \pm 1.48$ & $1.50 \pm 0.45$ & $4.95 \pm 1.55$ & $7.69 \pm 0.12$ & $1.07 \pm 0.29$ & $-1.49 \pm 0.04$ & $1.61 \pm 0.53$ \\
\hline UGC 1056 & A & $13000 \pm 2500$ & $13500 \pm 2600$ & $4.50 \pm 2.89$ & $3.26 \pm 1.56$ & $7.75 \pm 3.29$ & $7.89 \pm 0.15$ & $1.48 \pm 0.59$ & $-1.48 \pm 0.05$ & $2.57 \pm 1.14$ \\
\hline UGC 1056 & $\mathrm{~B}$ & $12600 \pm 900$ & $12700 \pm 900$ & $4.23 \pm 1.06$ & $5.43 \pm 1.04$ & $9.66 \pm 1.49$ & $7.98 \pm 0.06$ & $1.34 \pm 0.21$ & $-1.49 \pm 0.03$ & $3.16 \pm 0.52$ \\
\hline UGC 1176 & A & $12600 \pm 500$ & $12800 \pm 500$ & $3.40 \pm 0.47$ & $5.91 \pm 0.59$ & $9.31 \pm 0.75$ & $7.97 \pm 0.03$ & $1.29 \pm 0.11$ & $-1.40 \pm 0.02$ & $3.73 \pm 0.34$ \\
\hline NGC 784 & $\mathrm{~B}$ & $12900 \pm 600$ & $13400 \pm 700$ & $3.57 \pm 0.59$ & $4.70 \pm 0.58$ & $8.27 \pm 0.83$ & $7.92 \pm 0.04$ & $0.78 \pm 0.08$ & $-1.63 \pm 0.02$ & $1.94 \pm 0.22$ \\
\hline NGC 784 & A & $12500 \pm 500$ & $12400 \pm 500$ & $3.41 \pm 0.48$ & $7.20 \pm 0.78$ & $10.61 \pm 0.91$ & $8.03 \pm 0.04$ & $1.24 \pm 0.11$ & $-1.44 \pm 0.02$ & $3.87 \pm 0.36$ \\
\hline UGC 2716 & A & $12800 \pm 500$ & $13100 \pm 500$ & $2.96 \pm 0.42$ & $6.41 \pm 0.70$ & $9.37 \pm 0.82$ & $7.97 \pm 0.04$ & $0.99 \pm 0.09$ & $-1.47 \pm 0.02$ & $3.14 \pm 0.31$ \\
\hline NGC 2537 & $\mathrm{~A}$ & $10900 \pm 600$ & $9700 \pm 500$ & $8.85 \pm 1.94$ & $8.56 \pm 1.60$ & $17.4 \pm 2.5$ & $8.24 \pm 0.06$ & $7.40 \pm 0.99$ & $-1.07 \pm 0.02$ & $14.8 \pm 2.25$ \\
\hline NGC 2537 & $\mathrm{~B}$ & $11800 \pm 900$ & $11200 \pm 900$ & $5.26 \pm 1.48$ & $4.23 \pm 0.96$ & $9.49 \pm 1.76$ & $7.98 \pm 0.07$ & $5.13 \pm 0.88$ & $-1.00 \pm 0.02$ & $9.41 \pm 1.82$ \\
\hline UGC 4278 & $\mathrm{~B}$ & $13300 \pm 1000$ & $14200 \pm 1000$ & $2.53 \pm 0.61$ & $2.32 \pm 0.41$ & $4.85 \pm 0.73$ & $7.69 \pm 0.06$ & $0.57 \pm 0.09$ & $-1.63 \pm 0.03$ & $1.11 \pm 0.20$ \\
\hline UGC 4278 & $\mathrm{~A}$ & $13500 \pm 600$ & $14600 \pm 600$ & $2.01 \pm 0.27$ & $2.86 \pm 0.28$ & $4.87 \pm 0.38$ & $7.69 \pm 0.03$ & $0.52 \pm 0.05$ & $-1.58 \pm 0.03$ & $1.27 \pm 0.13$ \\
\hline NGC 2552 & $\mathrm{~A}$ & $11400 \pm 500$ & $10400 \pm 500$ & $5.27 \pm 0.88$ & $8.93 \pm 1.23$ & $14.19 \pm 1.52$ & $8.15 \pm 0.04$ & $3.57 \pm 0.37$ & $-1.16 \pm 0.02$ & $9.68 \pm 1.10$ \\
\hline UGC 4393 & B & $12100 \pm 1000$ & $11700 \pm 1000$ & $4.55 \pm 1.36$ & $6.92 \pm 1.64$ & $11.5 \pm 0.2$ & $8.06 \pm 0.07$ & $3.63 \pm 0.66$ & $-1.09 \pm 0.02$ & $9.39 \pm 1.82$ \\
\hline UGC 4393 & $\mathrm{C}$ & $12800 \pm 800$ & $13100 \pm 800$ & $5.80 \pm 1.22$ & $3.92 \pm 0.63$ & $9.72 \pm 1.37$ & $7.99 \pm 0.06$ & $3.25 \pm 0.42$ & $-1.24 \pm 0.02$ & $5.55 \pm 0.82$ \\
\hline UGC 5139 & A & $12800 \pm 800$ & $13000 \pm 800$ & $2.70 \pm 0.60$ & $5.65 \pm 0.95$ & $8.35 \pm 1.13$ & $7.92 \pm 0.05$ & $0.72 \pm 0.12$ & $-1.56 \pm 0.05$ & $2.30 \pm 0.41$ \\
\hline IC 559 & A & $12000 \pm 1400$ & $11500 \pm 1300$ & $5.63 \pm 2.31$ & $6.18 \pm 2.02$ & $11.81 \pm 3.07$ & $8.07 \pm 0.10$ & $1.74 \pm 0.46$ & $-1.47 \pm 0.05$ & $3.97 \pm 1.13$ \\
\hline UGC 5272 & A & $13300 \pm 500$ & $14100 \pm 200$ & $1.34 \pm 0.17$ & $6.12 \pm 0.22$ & $7.46 \pm 0.27$ & $7.87 \pm 0.02$ & $0.34 \pm 0.03$ & $-1.59 \pm 0.02$ & $1.92 \pm 0.12$ \\
\hline UGC 5340 & A & $15200 \pm 1200$ & $19400 \pm 1500$ & $0.47 \pm 0.11$ & $1.12 \pm 0.16$ & $1.59 \pm 0.19$ & $7.20 \pm 0.05$ & $0.09 \pm 0.02$ & $-1.60 \pm 0.08$ & $0.40 \pm 0.09$ \\
\hline UGC 5423 & A & $13700 \pm 500$ & $15300 \pm 500$ & $2.33 \pm 0.27$ & $3.55 \pm 0.24$ & $5.88 \pm 0.36$ & $7.77 \pm 0.03$ & $1.08 \pm 0.08$ & $-1.32 \pm 0.02$ & $2.82 \pm 0.21$ \\
\hline UGC 5423 & $\mathrm{~B}$ & $13400 \pm 1100$ & $14400 \pm 1200$ & $2.17 \pm 0.59$ & $4.37 \pm 0.87$ & $6.54 \pm 1.05$ & $7.82 \pm 0.06$ & $0.79 \pm 0.16$ & $-1.43 \pm 0.05$ & $2.43 \pm 0.48$ \\
\hline UGC 5797 & $\mathrm{~A}$ & $13200 \pm 1000$ & $14000 \pm 1000$ & $2.04 \pm 0.51$ & $7.06 \pm 1.29$ & $9.11 \pm 1.39$ & $7.96 \pm 0.06$ & & $-1.35 \pm 0.06$ & $4.11 \pm 0.84$ \\
\hline UGC 5923 & A & $14300 \pm 2500$ & $16600 \pm 3000$ & $4.04 \pm 2.22$ & $2.08 \pm 0.78$ & $6.12 \pm 2.35$ & $7.79 \pm 0.14$ & $1.97 \pm 0.67$ & $-1.30 \pm 0.04$ & $3.10 \pm 1.23$ \\
\hline NGC 3738 & A & $12100 \pm 900$ & $11800 \pm 800$ & $4.98 \pm 1.27$ & $6.00 \pm 1.21$ & $10.98 \pm 1.76$ & $8.04 \pm 0.06$ & $2.31 \pm 0.36$ & $-1.33 \pm 0.02$ & $5.14 \pm 0.86$ \\
\hline NGC 3738 & $\mathrm{~B}$ & $12500 \pm 1400$ & $12500 \pm 1400$ & $5.36 \pm 2.15$ & $5.39 \pm 1.67$ & $10.75 \pm 2.72$ & $8.03 \pm 0.10$ & $2.23 \pm 0.55$ & $-1.37 \pm 0.03$ & $4.57 \pm 1.21$ \\
\hline NGC 3741 & A & $13700 \pm 500$ & $15200 \pm 400$ & $1.81 \pm 0.21$ & $2.93 \pm 0.20$ & $4.74 \pm 0.29$ & $7.68 \pm 0.03$ & $0.42 \pm 0.04$ & $-1.61 \pm 0.03$ & $1.15 \pm 0.10$ \\
\hline UGC 6817 & $\mathrm{~A}$ & $14200 \pm 500$ & $16500 \pm 300$ & $0.94 \pm 0.10$ & $2.47 \pm 0.11$ & $3.41 \pm 0.15$ & $7.53 \pm 0.02$ & $0.26 \pm 0.02$ & $-1.53 \pm 0.03$ & $1.01 \pm 0.07$ \\
\hline NGC 4163 & A & $14800 \pm 2000$ & $18200 \pm 2500$ & $3.28 \pm 1.34$ & $0.34 \pm 0.09$ & $3.62 \pm 1.34$ & $7.56 \pm 0.14$ & $1.00 \pm 0.30$ & $-1.49 \pm 0.06$ & $1.17 \pm 0.47$ \\
\hline CGCG 269-049 & A & $14400 \pm 500$ & $17100 \pm 500$ & $1.00 \pm 0.11$ & $1.95 \pm 0.11$ & $2.96 \pm 0.16$ & $7.47 \pm 0.02$ & $0.20 \pm 0.02$ & $-1.57 \pm 0.03$ & $0.80 \pm 0.08$ \\
\hline UGC 7577 & A & $13100 \pm 900$ & $13700 \pm 900$ & $2.17 \pm 0.49$ & $7.10 \pm 1.18$ & $9.27 \pm 1.28$ & $7.97 \pm 0.06$ & $0.91 \pm 0.15$ & $-1.37 \pm 0.04$ & $3.96 \pm 0.67$ \\
\hline NGC 4449 & $\mathrm{C}$ & $11500 \pm 900$ & $10600 \pm 800$ & $6.92 \pm 1.99$ & $6.69 \pm 1.59$ & $13.62 \pm 2.54$ & $8.13 \pm 0.07$ & $3.16 \pm 0.55$ & $-1.33 \pm 0.02$ & $6.30 \pm 1.22$ \\
\hline NGC 4449 & B & $11300 \pm 600$ & $10400 \pm 600$ & $6.73 \pm 1.44$ & $7.79 \pm 1.39$ & $14.52 \pm 2.00$ & $8.16 \pm 0.06$ & $2.96 \pm 0.39$ & $-1.36 \pm 0.02$ & $6.30 \pm 0.91$ \\
\hline NGC 4449 & A & $10800 \pm 500$ & $9600 \pm 200$ & $6.36 \pm 1.16$ & $14.50 \pm 1.08$ & $20.87 \pm 1.59$ & $8.32 \pm 0.03$ & $2.50 \pm 0.28$ & $-1.39 \pm 0.02$ & $8.50 \pm 0.73$ \\
\hline UGC 7605 & A & $13400 \pm 2000$ & $15100 \pm 2200$ & $2.18 \pm 1.01$ & $2.44 \pm 0.80$ & $4.61 \pm 1.29$ & $7.66 \pm 0.11$ & $0.57 \pm 0.23$ & $-1.54 \pm 0.11$ & $1.32 \pm 0.53$ \\
\hline NGC 4656 & A & $12600 \pm 700$ & $12700 \pm 700$ & $1.20 \pm 0.22$ & $11.03 \pm 1.53$ & $12.23 \pm 1.55$ & $8.09 \pm 0.05$ & $0.27 \pm 0.11$ & $-1.66 \pm 0.14$ & $2.66 \pm 1.10$ \\
\hline UGC 8201 & A & $12900 \pm 900$ & $13600 \pm 900$ & $2.18 \pm 0.50$ & $4.07 \pm 0.69$ & $6.25 \pm 0.85$ & $7.80 \pm 0.06$ & $0.28 \pm 0.06$ & $-1.77 \pm 0.07$ & $1.06 \pm 0.30$ \\
\hline UGC 8508 & A & $13300 \pm 1100$ & $14300 \pm 1200$ & $1.82 \pm 0.50$ & $3.90 \pm 0.78$ & $5.72 \pm 0.92$ & $7.76 \pm 0.07$ & $0.34 \pm 0.08$ & $-1.60 \pm 0.07$ & $1.44 \pm 0.36$ \\
\hline UGC 8638 & A & $12600 \pm 500$ & $12800 \pm 300$ & $2.63 \pm 0.36$ & $6.73 \pm 0.40$ & $9.36 \pm 0.54$ & $7.97 \pm 0.02$ & $0.79 \pm 0.07$ & $-1.51 \pm 0.02$ & $2.88 \pm 0.20$ \\
\hline UGC 8638 & $\mathrm{~B}$ & $13000 \pm 500$ & $13500 \pm 500$ & $2.38 \pm 0.33$ & $5.82 \pm 0.60$ & $8.20 \pm 0.69$ & $7.91 \pm 0.03$ & $0.61 \pm 0.07$ & $-1.58 \pm 0.04$ & $2.17 \pm 0.26$ \\
\hline UGC 8837 & A & $12900 \pm 900$ & $13400 \pm 900$ & $4.76 \pm 1.13$ & $1.80 \pm 0.32$ & $6.56 \pm 1.18$ & $7.82 \pm 0.07$ & $2.12 \pm 0.31$ & $-1.35 \pm 0.02$ & $2.94 \pm 0.55$ \\
\hline NGC 5477 & A & $12800 \pm 500$ & $13000 \pm 200$ & $1.89 \pm 0.25$ & $7.10 \pm 0.30$ & $8.99 \pm 0.39$ & $7.95 \pm 0.02$ & $0.50 \pm 0.04$ & $-1.56 \pm 0.02$ & $2.45 \pm 0.14$ \\
\hline
\end{tabular}

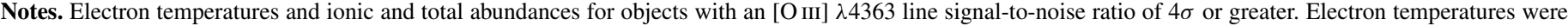
calculated using the $[\mathrm{O}$ III $](\lambda 4959+\lambda 5007) / \lambda 4363$ diagnostic line ratio.

expect for low-mass, low-luminosity galaxies. The abundances for the two additional objects outside of the LVL sample, UGC 4393 and UGC 10818, are discussed in Appendix B.

\section{THE $L-Z$ AND $M-Z$ RELATIONSHIPS}

The new "direct" oxygen abundances determined in this paper provide an opportunity to expand relationships previously limited by the reliability of empirical calibrations. In particular, these measurements allow us to re-examine the $L-Z$ and $M-Z$ relationships derived by L06, which are limited by small number statistics at the low luminosity end.

\subsection{The Total and "Select" Samples}

In the following, we analyze various samples based on both abundance measurement and distance measurement quality criteria. Specifically, we label the samples of galaxies with both direct oxygen abundance measurements and accurate distances as "Select." We observed 31 objects with [O III] $\lambda 4363$ detected at a strength greater than $4 \sigma$; this comprises our total sample. Our "direct" oxygen abundance measurements have relatively small errors, but comparisons to luminosity and stellar mass calculations require accurate distance determinations. This motivated further cuts from our sample to keep only objects with reliable distance determinations using the tip of the red giant branch (TRGB) or Cepheid variables (ceph), giving rise to our 13 object "Select" sample. In addition, the L06 data were updated with $4.5 \mu \mathrm{m}$ photometry from Dale et al. (2009) (to minimize the effects of aperture differences between the previous photometry and our own), distances from Dalcanton et al. (2009), and "direct" oxygen abundances from Croxall et al. (2009) when available. Those objects that passed the selection criteria were assembled into a similar "Select L06" sample of 14 objects. Other Local Volume objects presented in van Zee 
Table 5

Error Weighted Average Abundances

\begin{tabular}{|c|c|c|c|c|c|}
\hline Galaxy & $\begin{array}{l}\text { "Select" } \\
\text { Sample? }\end{array}$ & $\begin{array}{c}12+\log (\mathrm{O} / \mathrm{H}) \\
(\operatorname{dex})\end{array}$ & {$[\mathrm{O} \mathrm{III}] /[\mathrm{O} \mathrm{II}]$} & $\begin{array}{l}\log (\mathrm{N} / \mathrm{O}) \\
\quad(\operatorname{dex})\end{array}$ & $\begin{array}{c}\text { Previous Literature } \\
\text { Abundances? }\end{array}$ \\
\hline UGC 521 & & $7.67 \pm 0.05$ & $2.29 \pm 0.06$ & $-1.61 \pm 0.07$ & D: 12,13 \\
\hline UGC 695 & & $7.69 \pm 0.12$ & $0.50 \pm 0.01$ & $-1.49 \pm 0.04$ & \\
\hline UGC 1056 & & $7.97 \pm 0.06$ & $0.91 \pm 0.02$ & $-1.49 \pm 0.02$ & \\
\hline UGC 1176 & & $7.97 \pm 0.05$ & $1.59 \pm 0.05$ & $-1.40 \pm 0.02$ & \\
\hline NGC 784 & $\sqrt{ }$ & $7.97 \pm 0.06$ & $1.47 \pm 0.04$ & $-1.54 \pm 0.10$ & S: 2,6 \\
\hline UGC 2716 & & $7.97 \pm 0.05$ & $2.05 \pm 0.06$ & $-1.47 \pm 0.02$ & \\
\hline NGC 2537 & & $8.14 \pm 0.13$ & $0.63 \pm 0.02$ & $-1.04 \pm 0.04$ & S: 2, 21-23 \\
\hline UGC 4278 & & $7.69 \pm 0.05$ & $1.08 \pm 0.02$ & $-1.60 \pm 0.03$ & D: 16,17 \\
\hline NGC 2552 & & $8.15 \pm 0.05$ & $1.28 \pm 0.04$ & $-1.16 \pm 0.02$ & $\mathrm{~S}: 2,17,18$ \\
\hline UGC 4393 & & $8.02 \pm 0.05$ & $0.59 \pm 0.01$ & $-1.15 \pm 0.08$ & D: 16 \\
\hline UGC 5139 & $\sqrt{ }$ & $7.92 \pm 0.05$ & $1.95 \pm 0.06$ & $-1.56 \pm 0.05$ & D: 5,8 \\
\hline IC 559 & & $8.07 \pm 0.10$ & $0.90 \pm 0.03$ & $-1.47 \pm 0.05$ & \\
\hline UGC 5272 & & $7.87 \pm 0.05$ & $4.66 \pm 0.13$ & $-1.59 \pm 0.02$ & D: $6,10,11$ \\
\hline UGC 5340 & & $7.20 \pm 0.05$ & $3.26 \pm 0.13$ & $-1.60 \pm 0.08$ & D: 6,7 \\
\hline UGC 5423 & & $7.78 \pm 0.05$ & $1.77 \pm 0.03$ & $-1.33 \pm 0.04$ & D: 2,5 \\
\hline UGC 5797 & & $7.96 \pm 0.06$ & $3.48 \pm 0.10$ & $-1.35 \pm 0.06$ & \\
\hline UGC 5923 & & $7.79 \pm 0.14$ & $0.61 \pm 0.02$ & $-1.30 \pm 0.04$ & S: 9 \\
\hline NGC 3738 & $\sqrt{ }$ & $8.04 \pm 0.05$ & $1.02 \pm 0.03$ & $-1.34 \pm 0.02$ & D: $2,18-20$ \\
\hline NGC 3741 & $\sqrt{ }$ & $7.68 \pm 0.05$ & $1.78 \pm 0.05$ & $-1.61 \pm 0.03$ & $\mathrm{~S}: 2,3$ \\
\hline UGC 6817 & $\sqrt{ }$ & $7.53 \pm 0.05$ & $3.07 \pm 0.10$ & $-1.53 \pm 0.03$ & \\
\hline NGC 4163 & $\sqrt{ }$ & $7.56 \pm 0.14$ & $0.15 \pm 0.01$ & $-1.49 \pm 0.06$ & $\mathrm{~S}: 2$ \\
\hline CGCG 269-049 & $\sqrt{ }$ & $7.47 \pm 0.05$ & $1.13 \pm 0.03$ & $-1.57 \pm 0.03$ & D: 1 \\
\hline UGC 7577 & $\sqrt{ }$ & $7.97 \pm 0.06$ & $3.18 \pm 0.09$ & $-1.37 \pm 0.04$ & \\
\hline NGC 4449 & $\sqrt{ }$ & $8.26 \pm 0.09$ & $0.86 \pm 0.02$ & $-1.36 \pm 0.02$ & D: $17,19,20,24-26$ \\
\hline UGC 7605 & $\sqrt{ }$ & $7.66 \pm 0.11$ & $1.22 \pm 0.04$ & $-1.54 \pm 0.10$ & \\
\hline NGC 4656 & & $8.09 \pm 0.05$ & $8.39 \pm 0.35$ & $-1.66 \pm 0.14$ & S: 2,27 \\
\hline UGC 8201 & $\sqrt{ }$ & $7.80 \pm 0.06$ & $1.82 \pm 0.05$ & $-1.77 \pm 0.07$ & S: 8 \\
\hline UGC 8508 & $\sqrt{ }$ & $7.76 \pm 0.07$ & $2.21 \pm 0.06$ & $-1.60 \pm 0.07$ & $\mathrm{~S}: 2,4$ \\
\hline UGC 8638 & $\sqrt{ }$ & $7.95 \pm 0.05$ & $2.36 \pm 0.05$ & $-1.53 \pm 0.03$ & \\
\hline UGC 8837 & & $7.87 \pm 0.07$ & $0.36 \pm 0.01$ & $-1.43 \pm 0.03$ & D: 15 \\
\hline NGC 5477 & & $7.95 \pm 0.02$ & $0.54 \pm 0.01$ & $-1.56 \pm 0.02$ & D: 14 \\
\hline
\end{tabular}

Notes. For the 10 objects with multiple $\mathrm{H}_{\mathrm{II}}$ regions containing strong [O III] $\lambda 4363$, error weighted averages were used to determine best estimates of relative abundances and oxygen abundances. Column 2 highlights our "Select" sample. Columns 3-5 list the new "direct" oxygen abundances, ionization strengths, and nitrogen abundances relative to oxygen determined by this work. Average values were determined using a weight of $1 / \sigma_{i}^{2}$ for each component, where uncertainties represent the standard deviation of the weighted mean or the weighted dispersion, whichever is greater. Column 6 shows which objects have previous oxygen abundance determinations in the literature, where " $\mathrm{D}$ " is noted for objects with "direct" oxygen abundances, while "S" indicates objects with strong-line abundances. Note that we are providing "direct" oxygen abundances for the first time for 19 of these objects.

References. (1) Kniazev et al. 2003; (2) Moustakas \& Kennicutt 2006; (3) Gallagher \& Hunter 1989; (4) Vaduvescu et al. 2007; (5) Miller \& Hodge 1996; (6) Hunter \& Gallagher 1985; (7) Pustilnik et al. 2005; (8) Croxall et al. 2009; (9) Kewley et al. 2005; (10) Kinman \& Hintzen 1981; (11) Hopp \& Schulte-Ladbeck 1991; (12) van Zee et al. 1997b; (13) van Zee et al. 1997a; (14) Izotov et al. 2007b; (15) Liang et al. 2007; (16) Kniazev et al. 2004; (17) Izotov et al. 2006; (18) Hunter \& Hoffman 1999; (19) Hunter et al. 1982; (20) Martin 1997; (21) Engelbracht et al. 2008; (22) Gil de Paz et al. 2000b; (23) Gil de Paz et al. 2000a; (24) McCall et al. 1985; (25) Kobulnicky et al. 1999; (26) Sabbadin et al. 1984; (27) Matteucci \& Tosi 1985.

\& Haynes (2006) and Marble et al. (2010) were considered for an additional "Select" sample. Using the same criteria mentioned above, this provided 11 additional objects with "direct" abundances at a strength of $4 \sigma$ or greater and accurate TRGB distances. The 13 "Select" objects from this paper are noted in Table 5 and the properties of the additional objects taken from the literature are listed in Tables 6 and 7. Together these data sets made the final "Combined Select" sample comprising 38 objects with both secure distance (TRGB or ceph) and oxygen abundance determinations ([O III $] \lambda 4363>4 \sigma$ ). Note that we have 18 objects with accurate oxygen abundances that require accurate distances from TRGB observations in order to be elevated to the "Select" caliber. Of these, 13 have distances less than $8 \mathrm{Mpc}$, so their TRGB distances could be obtained with a relatively small investment of Hubble Space Telescope time.
Due to the wealth of $B$-band photometry available from previous studies, the majority of the sample has $B$-band absolute magnitude estimates. With the addition of Spitzer IRAC photometry, all members of the "Select" sample also have $4.5 \mu \mathrm{m}$ absolute magnitudes as determined by Dale et al. (2009). In the following sections, we discuss the low-luminosity portion of both the optical and NIR $L-Z$ relationships and the subsequently determined $M_{\star}-Z$ relationship, for our whole sample of "direct" oxygen abundances and a comparison to the filtered "Combined Select" sample.

\subsection{B-band L-Z Relationship}

In the top panel of Figure 3, we compare "direct" metallicities to corresponding $B$-band luminosities. Taking into consideration the errors on both quantities (cf., Press et al. 1992), we determine 
Additional "Select" Galaxies from the Literature

\begin{tabular}{|c|c|c|c|c|c|c|c|c|c|c|c|c|}
\hline Galaxy & $\begin{array}{l}\text { R.A. } \\
\text { (J2000) }\end{array}$ & $\begin{array}{l}\text { Decl. } \\
(\text { J2000) }\end{array}$ & $\begin{array}{l}F_{[4.5]} \\
(\mathrm{mJy})\end{array}$ & $\begin{array}{c}F_{K_{S}} \\
(\mathrm{mJy})\end{array}$ & $\begin{array}{c}D \\
(\mathrm{Mpc})\end{array}$ & Ref & $\begin{array}{c}M_{B} \\
(\mathrm{mag})\end{array}$ & $\begin{array}{l}M_{[4.5]} \\
(\mathrm{mag})\end{array}$ & $\begin{array}{l}M_{K_{S}} \\
\text { (mag) }\end{array}$ & $\begin{array}{l}(B-V)_{0} \\
\quad(\mathrm{mag})\end{array}$ & $\begin{array}{c}\log L_{[4.5]} \\
\left(L_{\odot}\right)\end{array}$ & $\begin{array}{c}\log M_{\star} \\
\left(M_{\odot}\right)\end{array}$ \\
\hline \multicolumn{13}{|c|}{ L06 "Select" Objects } \\
\hline WLM & 00:01:58.6 & $-15: 27: 12$ & 62.9 & 117 & $0.97 \pm 0.02$ & 1 & $-13.50 \pm 0.05$ & $-16.29 \pm 0.34$ & $-15.54 \pm 0.34$ & $0.46 \pm 0.03$ & $7.85 \pm 0.34$ & $7.19 \pm 0.34$ \\
\hline NGC 55 & 00:14:53.6 & $-39: 11: 48$ & 1390 & 2630 & $2.11 \pm 0.04$ & 2 & $-18.20 \pm 0.11$ & $-21.34 \pm 0.36$ & $-20.61 \pm 0.16$ & $0.55 \pm 0.08^{\mathrm{A}}$ & $9.87 \pm 0.35$ & $9.30 \pm 0.35$ \\
\hline UGC 00668 & 01:04:49.1 & 02:07:31 & 90.1 & 232 & $0.75 \pm 0.02$ & 1 & $-13.61 \pm 0.14$ & $-16.13 \pm 0.37$ & $-15.73 \pm 0.27$ & $0.40 \pm 0.04$ & $7.78 \pm 0.37$ & $7.14 \pm 0.37$ \\
\hline NGC 1705 & $04: 54: 13.7$ & $-53: 21: 41$ & 19.3 & 44.4 & $5.11 \pm 0.17$ & 3 & $-15.77 \pm 0.52$ & $-18.62 \pm 0.60$ & $-18.10 \pm 0.57$ & $0.38 \pm 0.18^{\mathrm{A}}$ & $8.78 \pm 0.60$ & $8.19 \pm 0.60$ \\
\hline NGC 2366 & $07: 28: 49.6$ & $69: 12: 32$ & 4.99 & 110 & $3.21 \pm 0.05$ & 2 & $-15.95 \pm 0.11$ & $-18.64 \pm 0.35$ & $-18.08 \pm 0.33$ & $0.32 \pm 0.05$ & $8.79 \pm 0.35$ & $8.15 \pm 0.35$ \\
\hline UGC 4305 & 08:19:09.0 & $70: 43: 28$ & 64.6 & 216 & $3.38 \pm 0.05$ & 2 & $-16.11 \pm 0.12$ & $-19.03 \pm 0.31$ & $-18.92 \pm 0.23$ & $0.19 \pm 0.06$ & $8.95 \pm 0.31$ & $8.48 \pm 0.31$ \\
\hline UGC 4459 & 08:34:07.6 & $66: 10: 39$ & 3.10 & 7.93 & $3.61 \pm 0.05$ & 2 & $-12.93 \pm 0.12$ & $-15.88 \pm 0.81$ & $-15.48 \pm 1.17$ & $0.46 \pm 0.08$ & $7.68 \pm 0.81$ & $7.15 \pm 0.81$ \\
\hline Leo A & $09: 59: 24.8$ & $30: 44: 49$ & 13.6 & 34.8 & $0.81 \pm 0.04$ & 4 & $-10.91 \pm 0.26$ & $-14.24 \pm 0.43$ & -13.82 & $0.24 \pm 0.06$ & $7.03 \pm 0.43$ & $6.58 \pm 0.43$ \\
\hline Sex B & 10:00:00.0 & $05: 19: 56$ & 36.1 & 136 & $1.39 \pm 0.04$ & 2 & $-13.54 \pm 0.16$ & $-16.47 \pm 0.38$ & $-16.49 \pm 0.25$ & $0.40 \pm 0.05$ & $7.92 \pm 0.37$ & $7.49 \pm 0.38$ \\
\hline Sex A & 10:11:00.7 & $-04: 41: 37$ & 24.9 & 62.2 & $1.38 \pm 0.05$ & 2 & $-13.62 \pm 0.19$ & $-16.05 \pm 0.39$ & $-15.62 \pm 0.49$ & $0.24 \pm 0.06$ & $7.75 \pm 0.39$ & $7.08 \pm 0.39$ \\
\hline UGC 5666 & $10: 28: 35.3$ & $68: 25: 53$ & 111 & 165 & $3.79 \pm 0.05$ & 2 & $-16.81 \pm 0.13$ & $-19.87 \pm 0.30$ & $-18.88 \pm 0.33$ & $0.34 \pm 0.06$ & $9.28 \pm 0.30$ & $8.62 \pm 0.30$ \\
\hline NGC 4214 & $12: 15: 39.0$ & $36: 19: 35$ & 224 & 488 & $3.03 \pm 0.05$ & 2 & $-17.15 \pm 0.10$ & $-20.15 \pm 0.36$ & $-19.58 \pm 0.17$ & $0.40 \pm 0.03$ & $9.39 \pm 0.36$ & $8.83 \pm 0.36$ \\
\hline UGC 8091 & $12: 58: 39.8$ & 14:13:06 & 2.29 & 8.40 & $2.08 \pm 0.02$ & 2 & $-11.76 \pm 0.07$ & $-14.35 \pm 0.35$ & $-14.34 \pm 0.97$ & $0.27 \pm 0.04$ & $7.07 \pm 0.35$ & $6.55 \pm 0.36$ \\
\hline IC 5152 & 22:02:41.6 & $-51: 17: 40$ & 103 & 306 & $1.97 \pm 0.07$ & 5 & $-15.47 \pm 0.03$ & $-18.37 \pm 0.34$ & $-18.13 \pm 0.15$ & $0.41 \pm 0.02$ & $8.68 \pm 0.34$ & $8.17 \pm 0.34$ \\
\hline \multicolumn{13}{|c|}{ Additional "Select" Objects } \\
\hline SMC & $00: 52: 44.0$ & $-72: 49: 42$ & 20.7 & & $0.056 \pm 0.002$ & 6 & $-16.04 \pm 0.20$ & $-8.89 \pm 0.34$ & & $0.45 \pm 0.10^{\mathrm{A}}$ & $4.89 \pm 0.46$ & \\
\hline UGC 00685 & 01:07:22.8 & 16:41:02 & 7.48 & 11.6 & $4.70 \pm 0.06$ & 5 & $-14.13 \pm 0.11$ & $-17.41 \pm 0.34$ & -16.46 & $0.60 \pm 0.09$ & $8.30 \pm 0.34$ & $7.71 \pm 0.34$ \\
\hline NGC 625 & 01:35:03.9 & $-41: 26: 14$ & 88.3 & 242 & $3.89 \pm 0.13$ & 7 & $-16.28 \pm 0.04$ & $-19.68 \pm 0.34$ & $-19.34 \pm 0.17$ & $0.59 \pm 0.02$ & $9.20 \pm 0.34$ & $8.80 \pm 0.34$ \\
\hline LMC & $05: 23: 34.6$ & $-69: 45: 22$ & 1.41 & $\ldots$ & $0.05 \pm 0.01$ & 8 & $-17.68 \pm 0.05$ & $-5.83 \pm 0.36$ & 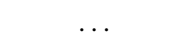 & $0.51 \pm 0.08^{\mathrm{A}}$ & $3.66 \pm 0.35$ & $\ldots$ \\
\hline UGC 4483 & 08:37:03.3 & 69:46:34 & 0.92 & 4.19 & $3.41 \pm 0.12$ & 2 & $-12.71 \pm 0.19$ & $-14.44 \pm 0.40$ & $-14.66 \pm 1.21$ & $0.15 \pm 0.05$ & $7.11 \pm 0.39$ & $6.42 \pm 0.40$ \\
\hline UGC 6541 & $11: 33: 28.8$ & $49: 14: 23$ & 3.59 & 12.6 & $3.89 \pm 0.52$ & 9 & $-13.51 \pm 0.06$ & $-16.20 \pm 0.34$ & $-16.14 \pm 0.64$ & $0.42 \pm 0.04$ & $7.81 \pm 0.34$ & $7.30 \pm 0.34$ \\
\hline UGCA 292 & $12: 38: 40.7$ & $32: 45: 41$ & 0.54 & 6.57 & $3.60 \pm 0.08$ & 2 & $-11.52 \pm 0.23$ & $-13.94 \pm 0.40$ & -15.27 & $0.07 \pm 0.14$ & $6.91 \pm 0.40$ & $6.68 \pm 0.40$ \\
\hline UGC 8651 & 13:39:53.9 & $40: 44: 26$ & 3.90 & 15.9 & $3.14 \pm 0.05$ & 2 & $-13.13 \pm 0.11$ & $-15.83 \pm 0.36$ & $-15.93 \pm 0.79$ & $0.36 \pm 0.06$ & $7.66 \pm 0.36$ & $7.19 \pm 0.36$ \\
\hline UGC 9128 & $14: 15: 56.8$ & $23: 03: 22$ & 2.43 & 9.25 & $2.21 \pm 0.07$ & 2 & $-12.12 \pm 0.18$ & $-14.55 \pm 0.38$ & $-14.58 \pm 0.88$ & $0.31 \pm 0.07$ & $7.15 \pm 0.38$ & $6.59 \pm 0.39$ \\
\hline UGC 9240 & $14: 24: 43.1$ & $44: 31: 37$ & 10.7 & 33.2 & $2.79 \pm 0.04$ & 2 & $-13.89 \pm 0.09$ & $-16.67 \pm 0.36$ & $-16.47 \pm 0.47$ & $0.43 \pm 0.06$ & $8.00 \pm 0.36$ & $7.47 \pm 0.36$ \\
\hline UGCA 442 & $23: 43: 46.3$ & $-31.57: 25$ & 7.51 & 13.4 & $4.27 \pm 0.53$ & 9 & $-14.34 \pm 0.62$ & $-17.21 \pm 0.71$ & $-16.41 \pm 1.14$ & $0.38 \pm 0.03$ & $8.21 \pm 0.71$ & $7.56 \pm 0.71$ \\
\hline
\end{tabular}

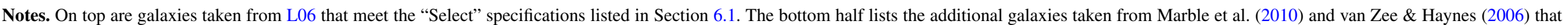

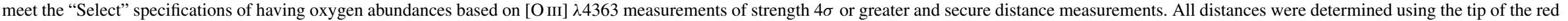
giant branch, with the exception of Leo A, which used Cepheid variables. $(B-V)_{0}$ optical colors are from L. van Zee et al. (2012, in preparation), unless otherwise noted.

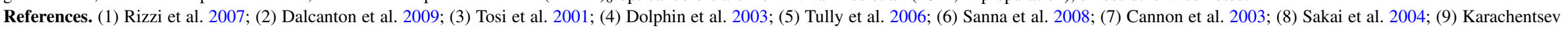
et al. 2003. 


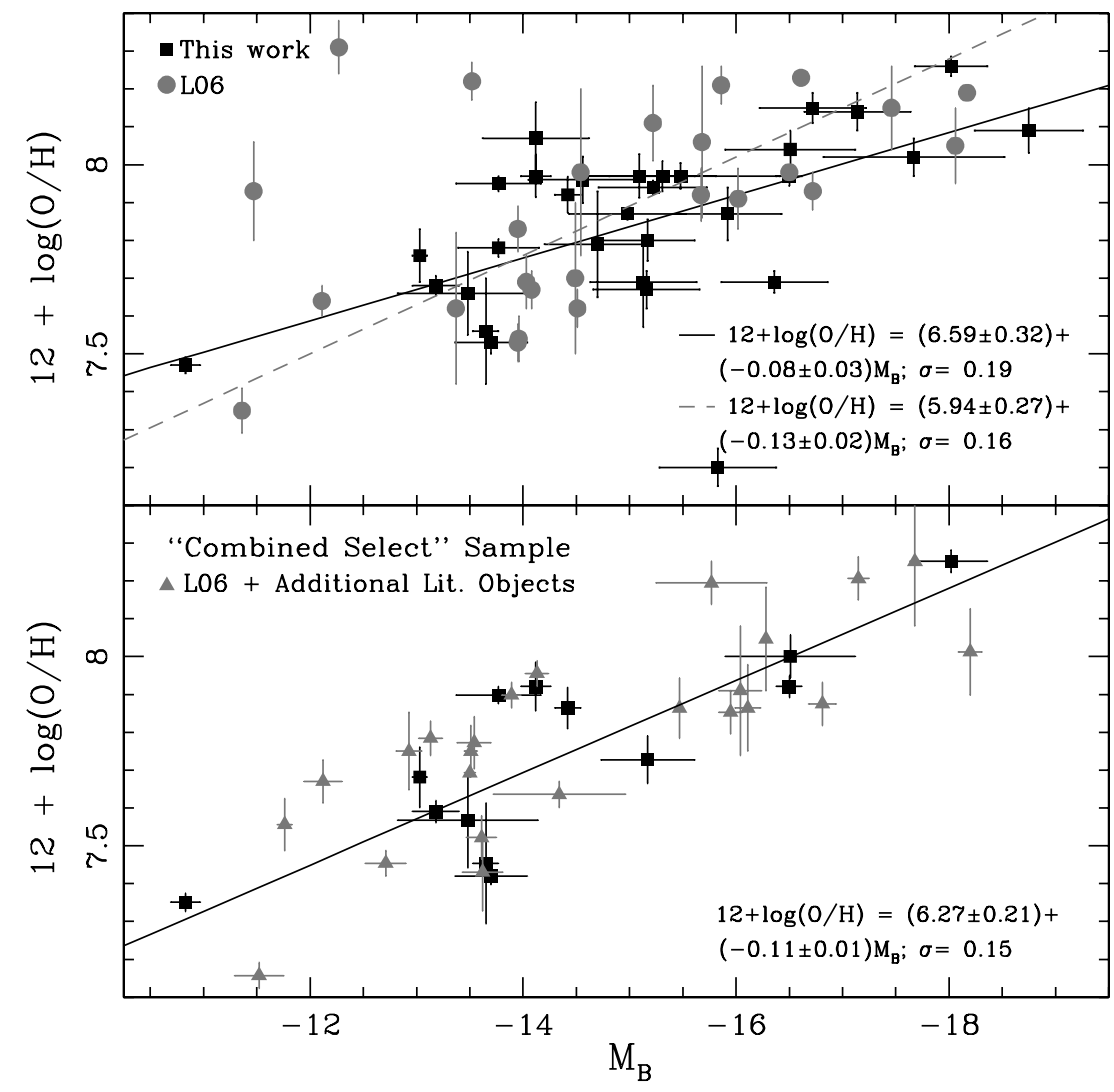

Figure 3. On the top, the optical luminosity-metallicity relationship is plotted for the 31 objects in the present sample with "direct" oxygen abundance measurements (squares). The solid black line represents the least-squares fit to these data. In comparison the original L06 dashed least-squares fit lies close to our line; in fact the slopes agree within the uncertainties. The updated L06 data are plotted (which are slightly offset from the original fit-see Section 6.3 for discussion of L06 data). On the bottom, the optical luminosity-metallicity relationship is improved by restricting our data to a "Combined Select" sample with "direct oxygen" abundances and reliable distance estimates (TRGB or ceph). The triangles represent the set of additional "Select" objects taken from L06, vZ06, and Marble et al. (2010), and the solid line is the least-squares fit to the total "Combined Select" sample.

the most likely linear fit to the data using the MPFITEXY routine (Williams et al. 2010), which depends, in turn, on the MPFIT package (Markwardt 2009). In this section, and those following, we provide the total scatter (intrinsic + observational) output from the MPFITEXY routine, which is essentially a weighted mean of the scatter of the data about the linear fit. In each case, we compare our results to that of L06, who also use a weighted dispersion routine.

The best fit to the 31 objects in the current sample with "direct" oxygen abundance measurements results in

$$
12+\log (\mathrm{O} / \mathrm{H})=(6.59 \pm 0.32)+(-0.08 \pm 0.03) M_{B},
$$

with a dispersion in $\log (\mathrm{O} / \mathrm{H})$ of $\sigma=0.19$. Updated data for the L06 sample (see Section 6.1) are also plotted and compared to the original least-squares best fit of L06.

The low-metallicity outlier at $12+\log (\mathrm{O} / \mathrm{H})=7.20$ is the blue compact dwarf UGC 5340, supporting its classification by previous work as one of the most metal-deficient star-forming galaxies (e.g., Izotov \& Thuan 2007a; Pustilnik et al. 2008b). However, Pustilnik et al. (2008b) note that its present distance could be significantly underestimated due to the large negative peculiar velocity in that region, which, if true, would result in an even larger discrepancy. Ekta et al. (2008) and Pustilnik et al. (2008a) have discussed the H I observations of UGC 5340 and concluded that it is likely undergoing a merger, which could explain, at least in part, its discrepant position from the $L-Z$ relationship. From $\mathrm{H}$ I observations of a sample of extremely metal-poor galaxies, Ekta \& Chengalur (2010) find that roughly half of these galaxies show evidence of interactions and conclude that the very low metallicities in these galaxies are due to recent infall of metal-poor gas (see also Lee et al. 2004). Thus, these galaxies do not lie on the $L-Z$ relationship defined by the average low-luminosity galaxy, and therefore UGC 5340 has not been included in the relationships of the "Combined Select" sample. ${ }^{16}$

In the lower panel of Figure 3, we plot the 38 objects in the "Combined Select" sample. The best fit is given by

$$
12+\log (\mathrm{O} / \mathrm{H})=(6.27 \pm 0.21)+(-0.11 \pm 0.01) M_{B} .
$$

with a resultant dispersion in $\log (\mathrm{O} / \mathrm{H})$ of $\sigma=0.15 .{ }^{17}$ Note that the luminosity error bars represent the error propagated from the uncertainty in the photometry and distances. This relationship agrees with that of L06 within errors. Additionally, the MPFITEXY routine allows us to estimate the intrinsic scatter by ensuring that $\chi^{2} /($ degrees or freedom $) \approx 1$. Using this tool, the intrinsic scatter in $\log (\mathrm{O} / \mathrm{H})$ for the $B$-band $L-Z$ relationship for the "Combined Select" sample is 0.13 dex, i.e., most of the scatter in this relationship is intrinsic.

\footnotetext{
16 At this time the Hi morphologies have not been analyzed for the LVL sample, so we cannot make predictions about the infall of unenriched gas for these galaxies.

17 Dispersion in $\log (\mathrm{O} / \mathrm{H})$ of the "Combined Select" sample increases to $\sigma=0.18$ if UGC 5340 is included.
} 
Table 7

Abundances for Additional "Select" Galaxies

\begin{tabular}{|c|c|c|c|}
\hline Galaxy & $\begin{array}{c}12+\log (\mathrm{O} / \mathrm{H}) \\
(\operatorname{dex})\end{array}$ & $\begin{array}{c}\log (\mathrm{N} / \mathrm{O}) \\
(\operatorname{dex})\end{array}$ & Reference \\
\hline WLM & $7.83 \pm 0.06$ & $-1.49 \pm 0.01$ & 1 \\
\hline NGC 55 & $8.05 \pm 0.10$ & $-1.26 \pm 0.05$ & 2 \\
\hline UGC 00668 & $7.62 \pm 0.05$ & $-1.51 \pm 0.10$ & 3 \\
\hline NGC 1705 & $8.21 \pm 0.05$ & $-1.75 \pm 0.06$ & 4 \\
\hline NGC 2366 & $7.91 \pm 0.05$ & $-1.17 \pm 0.26$ & 5 \\
\hline UGC 4305 & $7.92 \pm 0.10$ & $-1.52 \pm 0.11$ & 6 \\
\hline UGC 4459 & $7.82 \pm 0.09$ & $-1.32 \pm 0.17$ & 7 \\
\hline Leo A & $7.30 \pm 0.05$ & $-1.53 \pm 0.09$ & 8 \\
\hline Sex B & $7.53 \pm 0.05$ & $-1.49 \pm 0.06$ & 9 \\
\hline Sex A & $7.54 \pm 0.06$ & $-1.54 \pm 0.13$ & 10 \\
\hline UGC 5666 & $7.93 \pm 0.05$ & $-1.45 \pm 0.08$ & 7 \\
\hline NGC 4214 & $8.22 \pm 0.05$ & $-1.32 \pm 0.03$ & 11 \\
\hline UGC 8091 & $7.65 \pm 0.06$ & $-1.51 \pm 0.07$ & 8 \\
\hline IC 5152 & $7.92 \pm 0.07$ & $-1.05 \pm 0.12$ & 6 \\
\hline SMC & $7.96 \pm 0.15$ & $-1.55 \pm 0.15$ & 12 \\
\hline UGC 00685 & $8.00 \pm 0.03$ & $-1.45 \pm 0.08$ & 13 \\
\hline NGC 625 & $8.08 \pm 0.12$ & $-1.25 \pm 0.03$ & 14 \\
\hline LMC & $8.26 \pm 0.15$ & $-1.30 \pm 0.20$ & 12 \\
\hline UGC 4483 & $7.56 \pm 0.03$ & $-1.57 \pm 0.07$ & 13 \\
\hline UGC 6541 & $7.82 \pm 0.06$ & $-1.45 \pm 0.13$ & 15 \\
\hline UGCA 292 & $7.30 \pm 0.03$ & $-1.45 \pm 0.07$ & 9 \\
\hline UGC 8651 & $7.85 \pm 0.04$ & $-1.60 \pm 0.09$ & 13 \\
\hline UGC 9128 & $7.75 \pm 0.05$ & $-1.80 \pm 0.12$ & 16 \\
\hline UGC 9240 & $7.95 \pm 0.03$ & $-1.60 \pm 0.06$ & 13 \\
\hline UGCA 442 & $7.72 \pm 0.03$ & $-1.41 \pm 0.02$ & 14 \\
\hline
\end{tabular}

Notes. The top portion of the table lists the objects and their abundances which were included in the L06 sample. The bottom half lists additional objects found in the literature. All objects meet the "Select" sample criteria.

References. (1) Lee et al. 2005; (2) Tüllmann et al. 2003; (3) Lee et al. 2003a; (4) Lee \& Skillman 2004; (5) Saviane et al. 2008; (6) Lee et al. 2003b; (7) Croxall et al. 2009; (8) van Zee et al. 2006; (9) van Zee 2000; (10) Kniazev et al. 2005; (11) Kobulnicky \& Skillman 1996; (12) Russell \& Dopita 1990; (13) van Zee \& Haynes 2006; (14) Skillman et al. 2003 (15) Thuan \& Izotov 2005; (16) van Zee et al. 1997a.

\section{3. $4.5 \mu \mathrm{m}$ L-Z Relationship}

L06 found their $L-Z$ slope to be smaller in the NIR than in the optical and to contain less scatter. This result might be expected since luminosities in redder bands are less sensitive to dust extinction and SFRs than optical luminosities. However, these NIR luminosities are also vulnerable to stochastic effects from the high NIR luminosities of AGB stars. Following the motivation given in L06, we analyze the $4.5 \mu \mathrm{m} L-Z$ relationship.

In the top panel of Figure 4, we plot the $4.5 \mu \mathrm{m} L-Z$ relationship for our low-luminosity LVL sample. Our results are well matched to the luminosity-metallicity relationship for dwarf galaxies found by L06 (and corroborated by Marble et al. 2010). Using the MPFITEXY least-squares fit to our data, the resulting expression is

$$
12+\log (\mathrm{O} / \mathrm{H})=(6.37 \pm 0.33)+(-0.08 \pm 0.02) M_{[4.5]},
$$

with a standard deviation in $\log (\mathrm{O} / \mathrm{H})$ of $\sigma=0.18$. The original L06 least-squares fit and the updated L06 data are also plotted in Figure 4, displaying an equivalent slope, but with a notably smaller dispersion in $\log (\mathrm{O} / \mathrm{H})$ of only 0.12 . Note that while the two fits have the same slope, they are offset from one another by roughly $0.1 \mathrm{dex}$ in $\log (\mathrm{O} / \mathrm{H})$; this difference is within the error and can be attributed to the difference in samples and small sample size.

In the bottom panel of Figure 4, we have plotted the NIR $L-Z$ relationship for the "Combined Select" sample. A least-squares fit results in

$$
12+\log (\mathrm{O} / \mathrm{H})=(6.10 \pm 0.21)+(-0.10 \pm 0.01) M_{[4.5]}
$$

and produces a standard deviation of $\sigma=0.14{ }^{18}$ This is nearly identical to the standard deviation of $\sigma=0.15$ found for the "Combined Select" sample for the optical $L-Z$ relationship, and the slopes are the same within the uncertainties.

The intrinsic scatter in $\log (\mathrm{O} / \mathrm{H})$ for the $4.5 \mu \mathrm{m} L-Z$ relationship for the "Combined Select" sample is 0.11 dex. Since AGB stars can have significant impact on the NIR luminosities, we must consider the effect of stochastic sampling on the overall scatter of our relationship. However, since we find such a small scatter in the NIR $L-Z$ relationship it is unlikely to be due to AGB stars, which would normally drive the data to a larger dispersion. L06 determined dispersions in the optical and NIR $L-Z$ relationships of 0.161 and 0.122 , respectively. In comparison, the present work does not find a significant difference between the dispersions of the NIR and optical $L-Z$ relationships. However, the NIR intrinsic scatter in $\log (\mathrm{O} / \mathrm{H})$ is slightly smaller than the intrinsic scatter for the $B$-band $L-Z$ relationship for the "Combined Select" sample (0.11 versus 0.15 dex).

\section{4. $M_{\star}-Z$ Relationship}

The underlying relationship between mass and luminosity and the relative ease of measuring luminosities has allowed a widespread use of the $L-Z$ relationship. However, mass is thought to be more fundamentally related to metallicity (see, e.g., Tremonti et al. 2004), and so, when possible, metallicity is also investigated as a function of stellar mass. In order to examine the $M_{\star}-Z$ relationship, we need to estimate stellar masses in a self-consistent way. Although SED fitting is commonly used to determine individual masses, the necessary spectral and/or photometric components were not available to us for our entire "Combined Select" sample. Stellar mass can also be inferred from luminosity, where optical colors have been widely used to estimate $M / L$ ratios (e.g., Brinchmann \& Ellis 2000; Bell \& de Jong 2001). It is important to note the uncertainties in $M / L$ ratios that occur due to variations in the current SFR, which are most significant if galaxies have formed a substantial fraction $(>10 \%)$ of their stars in a recent episode. Near-IR magnitudes are often a better choice to characterize the galaxy luminosity because they are less sensitive than bluer bands to extinction and the age of the stellar population. The dominant emission in NIR wavelengths arises from the stellar populations (as opposed to dust) and is only marginally sensitive to recent star formation, but even so, NIR stellar $M / L$ ratios can vary by up to a factor of $\sim 2$ due to the SFR and stellar metallicity (Bell \& de Jong 2001). Furthermore, Lee et al. (2006a) found that although individual stellar masses can vary by as much as $\sim 0.5 \mathrm{dex}$ with $M / L$ model, the subsequent $M-Z$ relationship spanning four decades in stellar mass is nearly independent of the model chosen.

We chose to estimate stellar mass in a uniform manner from $4.5 \mu \mathrm{m}$ luminosity and $K-[4.5]$ and $B-K$ color following the method presented by L06:

$$
\log M_{\star}=\log \left(M_{\star} / L_{K}\right)+\left[\log L_{[4.5]}-0.4(K-[4.5])\right] .
$$

\footnotetext{
18 Dispersion in $\log (\mathrm{O} / \mathrm{H})$ of the "Combined Select" sample increases to
} $\sigma=0.22$ if UGC 5340 is included. 


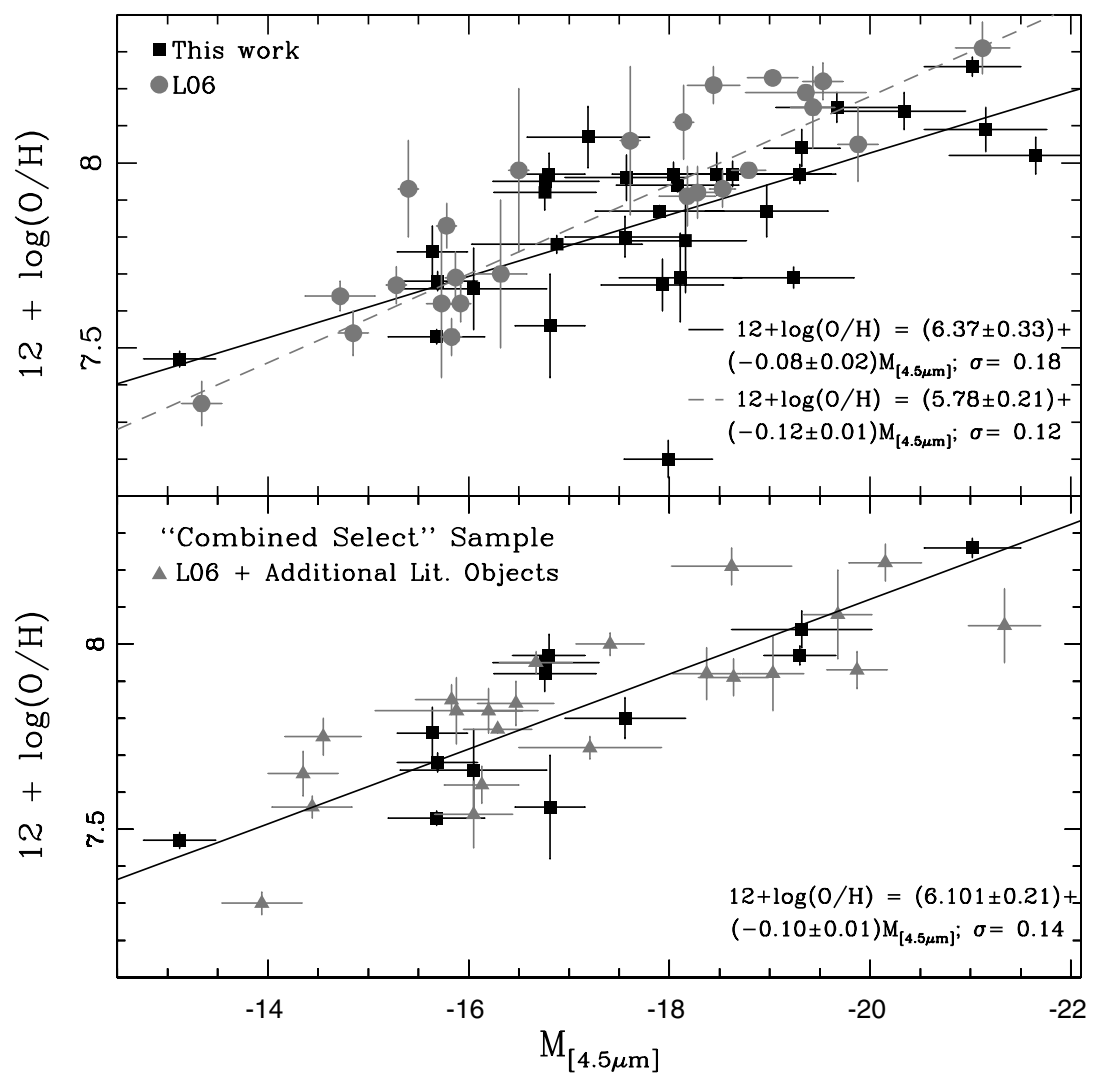

Figure 4. In the top panel, the NIR luminosity-metallicity relationship is plotted for the 31 objects in the present sample with "direct" oxygen abundance measurements (squares). The solid black line represents the least-squares fit to these data. In comparison, updated L06 data are plotted (circles), with the dashed original least-squares fit of L06 lying just above our line. Note that the updated data are slightly offset from the original fit (see Section 6.3 for discussion of L06 data). In the bottom panel is the NIR luminosity-metallicity relationship for the "Combined Select" sample, with "direct" oxygen abundances and reliable distance estimates (TRGB or ceph). By filtering our data in this way, the $L-Z$ relationship is strengthened.

L06 derived a mass-to-light ratio $\left(M_{\star} / L_{K}\right)$ as a linear function of $B-K$ color based on the Bruzual \& Charlot model with a Salpeter IMF. Note that there is a systematic uncertainty in NIR $M / L$ ratios of $\sim 0.2$ dex due to uncertainties in AGB evolution (e.g., Conroy \& Gunn 2010; Melbourne et al. 2012). Since $K_{s}$ photometry is available for the LVL sample (Dale et al. 2009), unlike the procedure of L06, the $B-K$ color was calculated directly (we assume $M_{K} \equiv M_{K_{s}}$ ). Based on the direct relationship between the ratio of luminosities and ratio of absolute magnitudes for two objects, we calculated monochromatic luminosities, $L_{[4.5]}$, assuming $M_{[4.5]} \simeq 3.3$ for the Sun (following the logic of L06). The $M_{\star}$ results are tabulated in Table 1.

In principle, mass estimates can be improved using SED fitting to broadband photometry which spans from the UV to the IR. B. D. Johnson et al. (2012, in preparation) have determined masses for the LVL galaxies using this method. Unfortunately, the broad wavelength coverage and associated analysis are not available for the entire LVL survey, including objects in our sample. There are $41 \mathrm{LVL}$ galaxies for which we have obtained new spectra or which have spectra in the literature with masses computed by B. D. Johnson et al. (2012, in preparation) to which we can compare our stellar masses determined from $4.5 \mu \mathrm{m}$ luminosities. We find an average difference of 0.23 dex in mass, or an offset of a factor of $\sim 2$, in the sense that the SED-derived masses are smaller and independent of luminosity or optical color. This difference can be accounted for by the use of different IMFs in the modeling (Salpeter IMF in Bell \& de Jong 2001 and Chabrier IMF in B. D. Johnson et al. 2012, in preparation).
Note that this average difference, as well as the dispersion of $\sigma=0.24$, is smaller than the typical uncertainty in our derived masses. Therefore, adopting these masses would not affect the slope of our derived $M-Z$ relationship. Because we do not have SED-derived masses for our entire "Combined Select" sample, we report the present relationship using the masses calculated here.

$M_{\star}-Z$ data are plotted in the top panel of Figure 5 in comparison to the updated L06 data and original $M_{\star}-Z$ relationship of L06. The best fit to our data,

$$
12+\log (\mathrm{O} / \mathrm{H})=(5.43 \pm 0.42)+(0.30 \pm 0.05) \log \left(M_{\star}\right),
$$

with a dispersion of $\sigma=0.21$, agrees, within errors, with the fit to the L06 data set. This dispersion is notably larger than the 0.12 dispersion in $\log (\mathrm{O} / \mathrm{H})$ found by $\mathrm{L} 06$. The mass error bars used here are the propagated errors from the $4.5 \mu \mathrm{m}$ luminosity, $K-$ [4.5] color, and mass-to-light ratio (where we substituted the uncertainty in $B-K$ color). Note that the contrast in dispersion of the two data sets is largely due to the different errors. L06 assumed the same errors for their mass determinations as their $4.5 \mu \mathrm{m}$ luminosities, whereas we incorporated the additional propagated error from the color terms. This difference accounts for the disparity in uncertainty.

On the bottom of Figure 5 we have plotted the "Combined Select" $M-Z$ data. Fitting the combined data set produces the least-squares linear fit,

$$
12+\log (\mathrm{O} / \mathrm{H})=(5.61 \pm 0.24)+(0.29 \pm 0.03) \log \left(M_{\star}\right),
$$




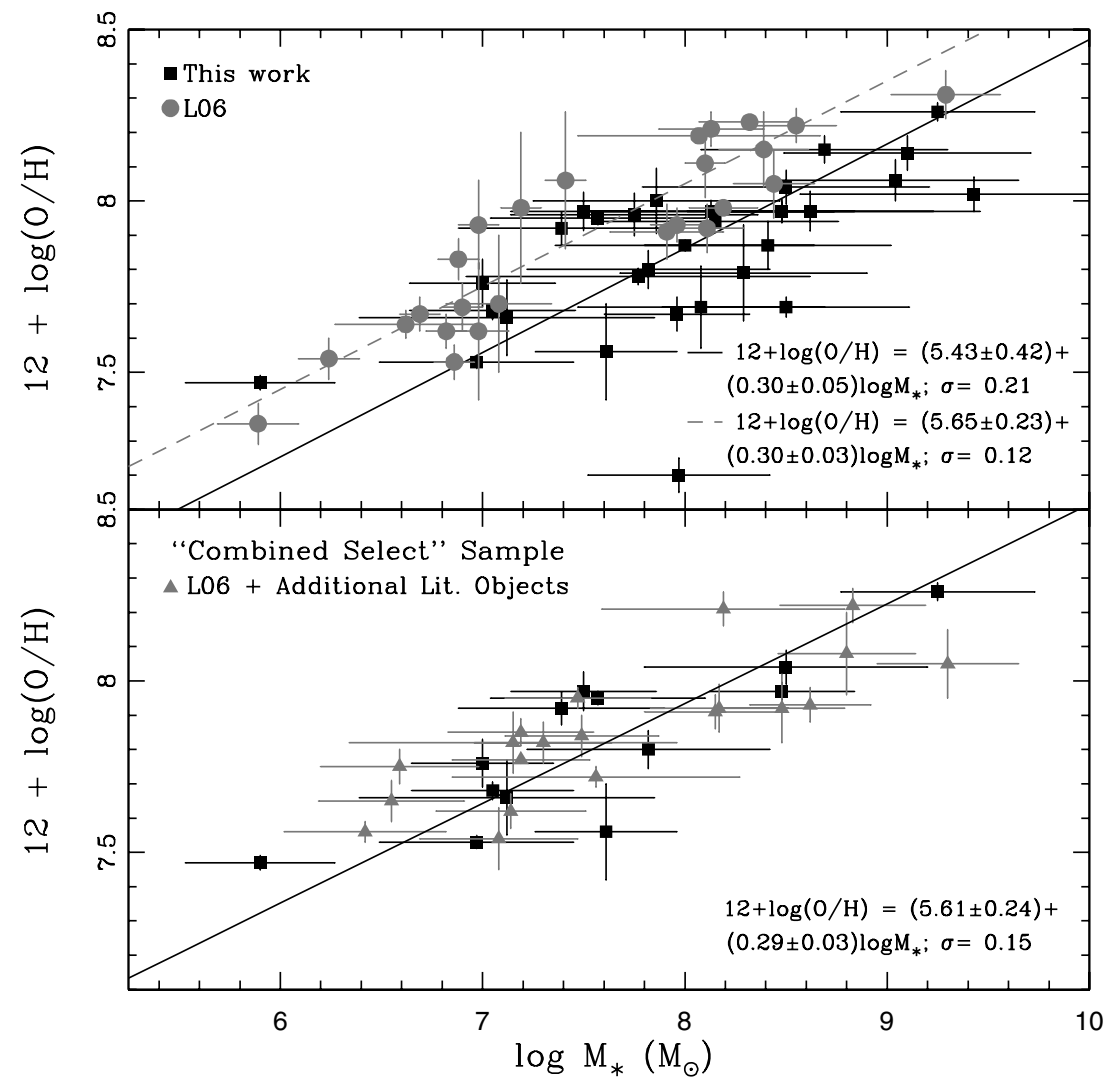

Figure 5. In the top panel the mass-metallicity relationship derived from NIR luminosities is plotted for the 31 objects in the present sample with "direct" oxygen abundance measurements (squares). The solid black line represents the least-squares fit to these data. In comparison, updated L06 data are plotted (circles). We have also plotted the original least-squares fit to the sample of objects from L06 (which is not significantly offset from the updated data; see Section 6.3 for discussion of L06 data). This dashed gray line is offset from our estimate of the best fit. In the lower panel is the mass-metallicity relationship for the "Combined Select" sample, with "direct" oxygen abundances and reliable distance estimates (TRGB or ceph).

with a standard deviation of $\sigma=0.15,{ }^{19}$ which is essentially equivalent to the dispersions of the "Combined Select" $L-Z$ data sets. The intrinsic scatter in $\log (\mathrm{O} / \mathrm{H})$ for the $M_{\star}-Z$ relationship for the "Combined Select" sample is 0.08 dex. This appears to be significantly smaller than the intrinsic scatter in $\log (\mathrm{O} / \mathrm{H})$ for the $4.5 \mu \mathrm{m} L-Z$ relationship for the "Combined Select" sample of 0.11 dex.

The dual effects of increasing the number of objects observed and selecting only objects with both reliable oxygen abundances and distances have resulted in a better characterization of the $L-Z$ and $M-Z$ relationships. In this work, we assume that a galaxy with an $\mathrm{H}$ II region of sufficiently high surface brightness to allow a $\lambda 4363$ measurement is a local property of the starforming region and not related to a characteristic property of the host galaxy. Thus, we do not believe our sample to be biased in terms of mass or galaxy type. Additionally, the observation that strong-line abundances of low-mass galaxies are consistent with the relationships derived here, albeit with increased scatter, supports this assumption. Therefore, the $L-Z$ and $M-Z$ relationships presented here should accurately represent low-mass galaxies in general. In high-mass galaxies, Tremonti et al. (2004) found a decrease in the dispersion in the $L-Z$ relationships as one went from $\sigma=0.16$ for the optical $B$ band to $\sigma=0.13$ for the longer wavelength $z$ band, and then an even smaller dispersion of $\sigma=0.10$ for the $M-Z$ relationship. The "Combined Select" data show a negligibly

\footnotetext{
19 Dispersion in $\log (\mathrm{O} / \mathrm{H})$ of the "Combined Select" sample increases to $\sigma=0.21$ if UGC 5340 is included.
}

smaller dispersion for the NIR $L-Z$ relationship compared to the $B$ band, and no similar decrease in dispersion for the $M-Z$ relationship.

\section{N/O RELATIVE ABUNDANCES}

The $\mathrm{N} / \mathrm{O}$ versus $\mathrm{O} / \mathrm{H}$ trend is well studied in galaxies of varying types. Vila Costas \& Edmunds (1993) presented a thorough overview of theoretical expectations and observations available at the time. A salient point is that $\mathrm{N}$ can be produced as both a primary and a secondary element and that the secondary component is expected to be delayed relative to oxygen and to dominate at high abundances. A typical scenario might be described by oxygen production in Type II supernovae being released $10 \mathrm{Myr}$ after star formation, whereas nitrogen forming in intermediate mass stars is not released until much later times ( $>10^{8}$ Myr; Kobulnicky \& Skillman 1996). Initially, N/O is expected to rapidly decrease as oxygen is returned to the ISM, but will gradually increase with time as nitrogen begins to be returned to the gas reservoir. Thus, in principle, the relative $\mathrm{N} / \mathrm{O}$ abundance can be used as a clock (e.g., Henry et al. 2000) to indicate the time since the most recent burst of star formation. Note that this effect is not expected if the SFR does not show significant variations (Mollá et al. 2006).

Table 5 lists the error weighted average $\mathrm{N} / \mathrm{O}$ values for our sample. The $\mathrm{N} / \mathrm{O}$ errors were determined by first adding in quadrature the error in flux of both $\left[\mathrm{O}_{\mathrm{II}}\right] \lambda 3727$ and $\left[\mathrm{N}_{\mathrm{II}}\right] \lambda 6584$, then adding this value in quadrature with the error in temperature of the low ionization zone. The most extreme values extend from $\log (\mathrm{N} / \mathrm{O})=-1.77$ to -1.00 , with an average of $\log (\mathrm{N} / \mathrm{O})=$ 

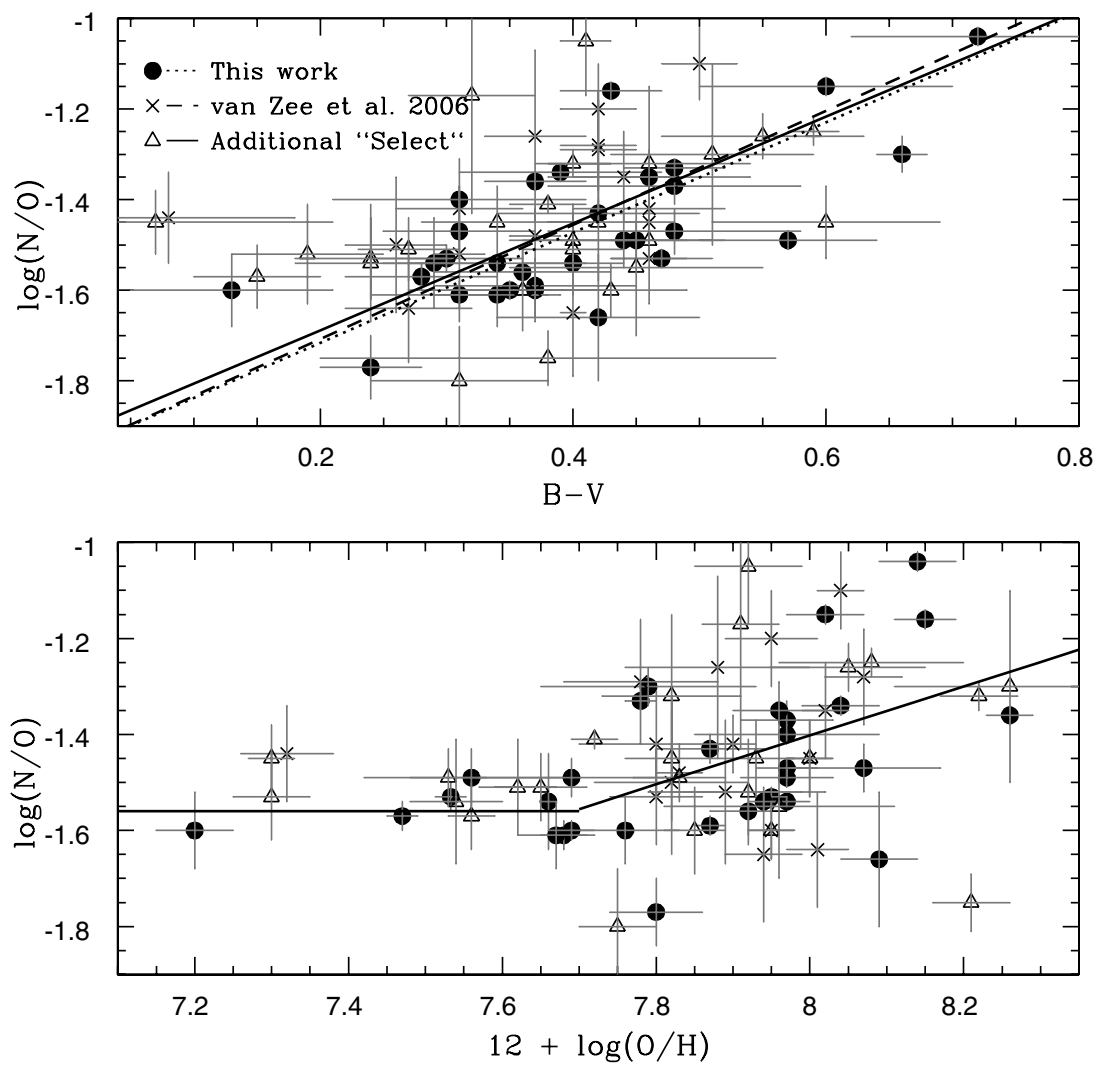

Figure 6. Relative $\mathrm{N} / \mathrm{O}$ abundance is depicted. The top panel compares $\log (\mathrm{N} / \mathrm{O})$ to $B-V$ color for objects of the present sample with [N II] observations (filled circles), the sample of vZ06 (crosses), and for the additional "Select" galaxies (triangles). The least-squares fit for this work is represented by the dotted line. The dashed line is the least-squares fit from vZ06. A solid line is shown for the literature combination of all three data sets: the present work, vZ06, and the additional values from the literature; our best estimate of the true relationship for the color range of $0.05 \lesssim B-V \lesssim 0.75$. Below $B-V=0.20$ the data diverge from the fit, suggesting this fit is most appropriate for the range of $0.20 \lesssim B-V \lesssim 0.75$. The bottom panel displays $\log (\mathrm{N} / \mathrm{O})$ vs. $\log (\mathrm{O} / \mathrm{H})$ for objects from this work, vZ06, and additional literature values. At values of $12+\log (\mathrm{O} / \mathrm{H}) \leqslant 7.7$, the $\mathrm{N} / \mathrm{O}$ values are relatively constant, consistent with the plateau associated with primary nitrogen return. Above this value of $\mathrm{O} / \mathrm{H}$, the scatter increases and the trend is to larger values of $\mathrm{N} / \mathrm{O}$ with the addition of secondary nitrogen.

-1.47 ; this is comparable to the isolated dwarf irregular sample examined by van Zee et al. (2006, hereafter vZ06), with an average $\log (\mathrm{N} / \mathrm{O})=-1.41$. We tested for a correlation of $\mathrm{N} / \mathrm{O}$ with reddening and found none, indicating an absence of bias in this regard.

The nine objects with multiple "direct" oxygen abundances provide the opportunity to study $\mathrm{N} / \mathrm{O}$ variations in individual dwarf galaxies. The average $\mathrm{N} / \mathrm{O}$ ratio dispersion of different $\mathrm{H}$ II regions in a given galaxy is only 0.08 dex, indicating that dwarf galaxies, despite appearing to be solid body rotators (Skillman et al. 1988), are well mixed (see also e.g., Roy $\&$ Kunth 1995). Other studies, such as the green pea galaxies analyzed by Amorín et al. (2010) and the nitrogen enriched dwarf galaxies analyzed by Pérez-Montero et al. (2011), find $\mathrm{N} / \mathrm{O}$ abundance dispersions or small gradients hypothesized to be a combination of outflows of enriched gas and inflows of metal-poor gas. Note that errors in $\mathrm{N} / \mathrm{O}$ account for the dispersion within four of the objects that have multiple $\mathrm{N} / \mathrm{O}$ measurements (UGC 1056, UGC 4278, NGC 3738, and NGC 4449), but not for five others (NGC 784, NGC 2537, UGC 4393, UGC 5423, and UGC 8638). For two of these objects (NGC 784 and UGC 4393) the differences in N/O are significant $(0.19$ and 0.15$)$. In these last two cases it could be that significant nitrogen enhancement has been detected, although not at the level of the well-studied galaxy NGC 5253 (e.g., Kobulnicky et al. 1997; López-Sánchez et al. 2012) or the more recently discovered N/O anomaly in MRK 996 (James et al. 2009).
vZ06 looked at several variables for their possible influence on $\mathrm{N} / \mathrm{O}$ abundance. In particular, they found a correlation between $\mathrm{N} / \mathrm{O}$ and color, in the sense that redder galaxies have higher $\mathrm{N} / \mathrm{O}$ as one might expect from time-delayed $\mathrm{N}$ release. In the top panel of Figure $6, \log (\mathrm{N} / \mathrm{O})$ is plotted versus $B-V$ color for objects of our sample with "direct" abundances and measurable $\left[\mathrm{N}_{\mathrm{II}}\right] /\left[\mathrm{O}_{\mathrm{II}}\right]$ abundances. Similar to vZ06, we find a fairly steep increase in $\mathrm{N} / \mathrm{O}$ with redder color (demonstrated by the dotted least-squares fit):

$$
\log (\mathrm{N} / \mathrm{O})=(-1.96 \pm 0.12)+(1.22 \pm 0.26) \times(B-V),
$$

with a dispersion of $\sigma=0.13$. In fact, the two groupings of points are visually consistent with one another. When the additional objects from the literature are added to the plot, the least-squares fit over $0.05 \lesssim B-V \lesssim 0.75$ to all of the data is

$$
\log (\mathrm{N} / \mathrm{O})=(-1.92 \pm 0.08)+(1.18 \pm 0.19) \times(B-V),
$$

which agrees well with the relationship found by vZ06. Below $B-V=0.10$ there are two objects with discrepantly large N/O values. Therefore, we suggest this fit is most appropriate for the range of $0.20 \lesssim B-V \lesssim 0.75$. Note the appearance of significant scatter in this figure. We calculate a dispersion in $\log (\mathrm{N} / \mathrm{O})$ of $\sigma=0.14 \mathrm{dex}$, with an estimated intrinsic scatter of 0.10 dex.

Additionally, the bottom panel of Figure 6 shows $\log (\mathrm{N} / \mathrm{O})$ plotted versus $12+\log (\mathrm{O} / \mathrm{H})$ for the same sample. Above $12+$ $\log (\mathrm{O} / \mathrm{H}) \approx 7.7$ a trend of $\mathrm{N} / \mathrm{O}$ increasing with $\mathrm{O} / \mathrm{H}$ is evident, 
despite the large scatter. For $12+\log (\mathrm{O} / \mathrm{H}) \geqslant 7.7$, the best fit to our data yields

$$
\log (\mathrm{N} / \mathrm{O})=(-5.49 \pm 1.36)+(0.51 \pm 0.17) \times[12+\log (\mathrm{O} / \mathrm{H})]
$$

with a dispersion of $\sigma=0.16$, where the estimated intrinsic scatter is 0.14 dex. With an increasing slope, this would be indicative of secondary $\mathrm{N}$ production in this region. Garnett (1990) proposed that much of the scatter in the $12+\log (\mathrm{O} / \mathrm{H})$ versus $\log (\mathrm{N} / \mathrm{O})$ relationship could be explained by the time delay between producing oxygen and secondary nitrogen.

For the systems with $12+\log (\mathrm{O} / \mathrm{H}) \leqslant 7.7$, in agreement with previous studies, there is little trend in $\mathrm{N} / \mathrm{O}$ with $\mathrm{O} / \mathrm{H}$. We have calculated a weighted mean in $\mathrm{N} / \mathrm{O}$ using the IDL routine MPFITEXY with the added constraint of setting the slope to zero for the points below $12+\log (\mathrm{O} / \mathrm{H})=7.7$. For our eight new observations, the weighted mean is $\log (\mathrm{N} / \mathrm{O})=-1.56$ with a standard deviation of 0.05 . For the nine observations from the literature, the weighted mean is $\log (\mathrm{N} / \mathrm{O})=-1.51$ with a standard deviation of 0.04 . For the two sets together we obtain $\log (\mathrm{N} / \mathrm{O})=-1.56$ with a standard deviation of 0.05 . Of this dispersion, the intrinsic scatter is predicted to be 0.02 , so observational scatter may play a large role in determining the observed scatter in this relationship. In most previous studies, no correlation is noted between $12+\log (\mathrm{O} / \mathrm{H})$ and the relative $\mathrm{N} / \mathrm{O}$ abundance at low oxygen abundances, where nitrogen is expected to behave like a primary nucleosynthesis element. Together the new observations are consistent with the trends in N/O with O/H observed by Vila Costas \& Edmunds (1993), Lee et al. (2004), van Zee \& Haynes (2006), Mollá et al. (2006), and Liang et al. (2006).

\section{DISCUSSION}

\subsection{The L-Z and $M-Z$ Relations for Low-luminosity Galaxies}

The dual effects of increasing the sample size and selecting only objects with both reliable oxygen abundances and distances have resulted in an improved characterization of the $L-Z$ and $M-Z$ relationships. In high-mass galaxies, Tremonti et al. (2004) found a decrease in the dispersion in the $L-Z$ relationship as one went from the optical $B$ band $(\sigma=0.16)$ to the longer wavelength $z$ band $(\sigma=0.13)$, and an even smaller dispersion for the $M-Z$ relationship ( $\sigma=0.10$ ). The present data show only a slightly smaller dispersion for the NIR $L-Z$ relationship ( $\sigma=0.14)$ compared to the $B$ band $(\sigma=0.15)$, but no similar decrease in dispersion for the $M-Z$ relationship ( $\sigma=0.15$ ). However, our estimates of the intrinsic scatter in the three relationships do show a decreasing trend in the sense that the intrinsic scatter of the $B$-band $L-Z$ relationship is largest ( $\sigma=0.13$ ), followed by the NIR $L-Z$ relationship ( $\sigma=0.12$ ), then the $M-Z$ relationship ( $\sigma=0.08$ ). While this trend could be an artifact of how the errors are estimated for the three different parameters, it is interesting that it follows the same pattern observed in the larger spiral galaxies. Perhaps what is most remarkable is the small intrinsic scatter in all three relationships. When averaging the light over an entire galaxy, as done in Tremonti et al. (2004), one might expect relatively low dispersions. However, oxygen abundances derived from spectroscopic apertures only covering a fraction of the galaxy will be biased if radial gradients exist (e.g., Moustakas et al. 2012). Therefore, one might expect much larger dispersions when observing individual $\mathrm{H}$ II regions, yet this is not the case observed in most dwarf galaxies, as they have been shown to be relatively chemically homogeneous (e.g., Croxall et al. 2009).
The $L-Z$ and $M-Z$ relationship slopes determined for the "Combined Select" sample are similar to those found in previous studies (e.g., Tremonti et al. 2004; Lee et al. 2006a). For large galaxies, a different slope may apply as galaxies higher in mass and luminosity contain more metals and dust (e.g., Rosenberg et al. 2006) causing them to appear underluminous. For smaller, less luminous galaxies, even with the present sample included, the number of galaxies meeting our "Select" criteria is still relatively small. This limitation could affect our measurements of the scatter, but it appears that these relationships have intrinsically smaller dispersions. The evolutionary paths of dwarfs are still poorly understood, making the source of this inherent variation unclear. Some studies argue for the importance of gas infall and outflows (e.g., Garnett 2002), whereas others point to star formation efficiencies (e.g., Lequeux et al. 1979; Brooks et al. 2007), and variations in IMFs (e.g., Köppen et al. 2007). Still other studies have also seen significant scatter at low stellar masses (see for example Tremonti et al. 2004; Amorín et al. 2010).

Amorín et al. (2010) suggest that inherent variation in the $L-Z$ and $M-Z$ relations could result from these objects being relatively young and thus they may still be converting large amounts of cold gas into stars. If these young galaxies have not had enough time for several generations of star formation to produce massive AGB stars, then we would expect very little absorption due to dust. The relative uniformity between the dispersions of the $L-Z$ and $M-Z$ relationships and between the slopes of the optical and near-IR $L-Z$ relationships is consistent with this idea, suggesting no more absorption in the optical than in the near-IR, and thus very little dust is present in these low-luminosity galaxies. The fact that the scatter in the $L-Z$ and $M-Z$ relationships is small suggests that AGB stars do not play as significant a role in determining the scatter in the NIR $L-Z$ relationship for low-mass galaxies. In fact, in our sample it seems that AGB stars are balanced out by the effects of star formation histories. Whatever the actual source of the scatter may be, since we used the most reliable oxygen abundances and distance estimates possible in constructing the $L-Z$ and $M-Z$ relationships, it appears that the dispersion for this sample is real as it is larger than observational errors. However, the "young galaxy" hypothesis faces other observational challenges.

\subsection{N/O and the Young Galaxy Hypothesis}

Garnett (1990) first showed that the N/O ratio in lowmetallicity star-forming galaxies is relatively constant as a function of $\mathrm{O} / \mathrm{H}$ (with a mean value of $\log (\mathrm{N} / \mathrm{O})=-1.46_{-0.13}^{+0.10}$ ) for these "plateau" objects. Later, Izotov \& Thuan (1999) drew attention to the plateau with small dispersion in $\log (\mathrm{N} / \mathrm{O})$ $(-1.60 \pm 0.02)$ in extremely metal-poor $(12+\log (\mathrm{O} / \mathrm{H}) \leqslant 7.6)$ blue compact dwarf galaxies. They proposed that the absence of time-delayed production of $\mathrm{N}$ (and $\mathrm{C}$ ) is consistent with the scenario that extremely metal-poor galaxies are now undergoing their first burst of star formation, and that they are therefore young, with ages not exceeding $40 \mathrm{Myr}$. They further argued that if this were true, then this would argue against the commonly held belief that $\mathrm{C}$ and $\mathrm{N}$ are produced by intermediate-mass stars at very low metallicities (as these stars would not have yet completed their evolution in these lowest metallicity galaxies). Nava et al. (2006) revisited the observed N/O plateau with a large set of objects and determined a mean value for the $\mathrm{N} / \mathrm{O}$ plateau of -1.43 with a standard deviation of ${ }_{-0.084}^{+0.071}$. They further concluded from a $\chi^{2}$ analysis that only a small fraction of the observed scatter in $\mathrm{N} / \mathrm{O}$ is intrinsic. 
From the bottom panel of Figure 6 , we see that the sample assembled here also shows a plateau in $\mathrm{N} / \mathrm{O}$ of $\log (\mathrm{N} / \mathrm{O})=$ $-1.56 \pm 0.05$. The level of the plateau in our data is slightly lower than found by Nava et al. (2006), but agrees fairly well with that found by Izotov \& Thuan (1999). While the observed dispersion is larger than that found for the blue compact dwarfs by Izotov \& Thuan (1999), the intrinsic dispersion agrees well for the two samples. Clearly the relatively constant $\mathrm{N} / \mathrm{O}$ value is a common characteristic of dwarf star-forming galaxies, and not just those undergoing a current burst of star formation. van Zee et al. (2006) demonstrated that Leo A, with $12+\log (\mathrm{O} / \mathrm{H})=$ $7.38 \pm 0.10$ and $\log (\mathrm{N} / \mathrm{O})=-1.53 \pm 0.09$, and GR 8 , with $12+\log (\mathrm{O} / \mathrm{H})=7.65 \pm 0.06$ and $\log (\mathrm{N} / \mathrm{O})=-1.51 \pm 0.07$, which are not blue compact dwarf galaxies, are consistent with this plateau in $\log (\mathrm{N} / \mathrm{O})$ at low values of $\mathrm{O} / \mathrm{H}$. However, both Leo A and GR 8 have detailed star formation histories derived from Hubble Space Telescope observations of their resolved stars which clearly show that the bulk of their star formation occurred well before the last $40 \mathrm{Myr}$ (Tolstoy et al. 1998; Cole et al. 2007; Dohm-Palmer et al. 1998; Weisz et al. 2011). In fact, Weisz et al. (2011) show, from a nearly volume-limited sample, that the majority of dwarf galaxies formed the bulk of their stellar mass prior to $z \sim 1$, regardless of current morphological type. Since the low mass, metal-poor galaxies in the present sample and works cited appear to have nearly the same value of $\mathrm{N} / \mathrm{O}$, regardless of whether they have a current burst of star formation, it would seem that the young galaxy hypothesis is not a valid explanation for the plateau in N/O at low metallicity.

If the plateau in $\mathrm{N} / \mathrm{O}$ is not due to young galaxy ages, what is its cause? Clearly nitrogen is behaving as a primary element at low metallicities. Henry et al. (2006) considered various scenarios and concluded that a wide range were consistent with the observations. At this point, a definitive explanation for the $\mathrm{N} / \mathrm{O}$ plateau appears elusive.

\subsection{Best Estimate of Abundances}

Determining an accurate and reliable oxygen abundance for an individual $\mathrm{H}$ II region depends on measuring the combination of bright nebular and faint auroral emission lines (the "direct" method). Many studies have emphasized that a "direct" abundance is not without systematic uncertainties. Specifically, due to the high temperature sensitivity of the "direct" method, inhomogeneous temperature distributions will lead to abundance underestimates. The uncertainty in the absolute oxygen abundance determination by this method is $\sim 0.1 \mathrm{dex}$, but the error in relative metallicities is likely to be $\ll 0.1$ dex (Kewley \& Ellison 2008). However, Bresolin (2007) warns that $T_{e}$-based determinations only provide a lower limit if the temperature fluctuations are substantial.

In the absence of a temperature-sensitive auroral line detection, a mix of strong emission lines are used as a proxy for metallicity (strong-line methods: empirical, semi-empirical, and theoretical calibrations). Strong-line calibrations are limited by sample selection effects, potentially making them appropriate for ranking objects on a single scale, but not useful for determining an absolute metallicity as the various methods do not converge (see, e.g., Yin et al. 2007; Kewley \& Ellison 2008; Bresolin et al. 2009; Berg et al. 2011). If a strong-line method must be used, Stasińska (2010) recommends only using a strongline method for nebulae having the same properties as those of the calibration sample.

Oey \& Shields (2000), van Zee \& Haynes (2006), van Zee et al. (2006), Yin et al. (2007), Kewley \& Ellison (2008), Pérez-
Montero \& Contini (2009), Amorín et al. (2010), Moustakas et al. (2010), and others have investigated several strong-line calibrations including the O3N2 method, the N2 method, and the $R_{23}$ index, finding inconsistencies between methods that were largely related to variations in the hardness of the ionizing radiation field, nitrogen abundance, and/or age of the stellar cluster. There are several strong-line methods to chose from, but when compared they all have similar uncertainties of $0.1-0.2$ dex and discrepancies between them as large as 0.6 dex (e.g., Liang et al. 2006; Bresolin 2007; Yin et al. 2007; Kewley \& Ellison 2008). Improvements have been made in strong-line calibrations by the introduction of photoionization models to simultaneously fit the most prominent emission lines (e.g., Tremonti et al. 2004; Brinchmann et al. 2004). However, Yin et al. (2007) found the MPA/JHU simultaneous line fitting SDSS abundances determined from the Charlot photoionization models overestimate oxygen abundances by $\sim 0.34$ dex compared to direct abundances. They postulate the difference to be due to the model's treatment of the onset of secondary nitrogen production, and thus it could be eliminated with improved modeling. One possible exception is the ONS calibration of Pilyugin et al. (2010), for which they find deviations from $T_{e}$-based oxygen abundances of just $\sim 0.075$ dex.

Here, we investigate a subset of strong line abundances for our objects with "direct" abundances. Following the methodology of Berg et al. (2011), we calculated oxygen abundances from their strong lines for the 31 objects with "direct" abundances listed in Table 5. We determined abundances using the $R_{23}$ calibration of McGaugh (1991), the ONS calibration of Pilyugin et al. (2010), and the N2 and O3N2 calibrations updated by Pérez-Montero \& Contini (2009, hereafter PMC09). The $R_{23}$ calibration of McGaugh (1991) produces a bi-valued solution, so to discriminate between the two branches McGaugh (1994), van Zee et al. (1998), and others advised using the ratio $I([\mathrm{~N}$ II $] \lambda 6584) / I([\mathrm{O}$ II $] \lambda 3727)$. McGaugh (1994) suggested that $[\mathrm{NII}] /\left[\mathrm{O}_{\mathrm{II}}\right]$ is approximately $<0.1$ for low abundances and $>0.1$ for high abundances, giving a rough distinction between lower and upper branches. Using this distinction, we selected the appropriate branch calibration for each object. Note that for metal-poor objects with enhanced nitrogen, [N $\mathrm{II}] /\left[\mathrm{O}_{\mathrm{II}}\right]$ becomes a biased discriminator (e.g., Yin et al. 2007; Berg et al. 2011; Pérez-Montero et al. 2011). In a similar fashion, the ONS method of Pilyugin et al. (2010) requires two discriminators, $[\mathrm{N}$ II $]$ and $[\mathrm{N} I I] /\left[\mathrm{S}_{\mathrm{II}}\right]$, to distinguish between three classes of $\mathrm{H}$ II regions.

We followed Berg et al. (2011) and assumed $T_{\mathrm{e}}=1.25 \times$ $10^{4} \mathrm{~K}$ to examine $\mathrm{N} / \mathrm{O}$ ratios and calculate abundances with the $\mathrm{N} 2$ and $\mathrm{O} 3 \mathrm{~N} 2$ calibrations of PMC09. This correction may be important for NGC 2537 and UGC 4393, which appear to have somewhat discrepant nitrogen abundances (nitrogen enrichment for $\log (\mathrm{N} / \mathrm{O})>-1.0)$. The other objects in this sample have average N/O ratios for their masses (see, e.g., Berg et al. 2011). The results are tabulated in Table 8 .

The mean offsets and dispersions relative to the direct abundances are calculated and given at the bottom of Table 8 . Table 8 shows that all four methods have significant dispersions, with the ONS method showing the smallest dispersion (although larger than anticipated) and the O3N2 method having the largest. The ONS method also has the smallest mean offset. Figure 7 presents a plot of differences between the $R_{23}$ and ONS method abundances and the direct abundances as a function of abundance. This illustrates the results of Table 8, that the ONS method has a smaller dispersion and a smaller mean offset 
Table 8

Strong-line Oxygen Abundances for Our "Direct" Detection Galaxies

\begin{tabular}{|c|c|c|c|c|c|}
\hline \multirow[b]{2}{*}{ Galaxy } & \multirow[b]{2}{*}[\mathrm{N}\text{II}]{$/\left[\mathrm{O}_{\mathrm{II}}\right]$} & \multicolumn{4}{|c|}{$12+\log (\mathrm{O} / \mathrm{H})$} \\
\hline & & $R_{23}$ & ONS & $\mathrm{N} 2$ & $\mathrm{O} 3 \mathrm{~N} 2$ \\
\hline UGC 521 A & 0.03 & $7.90(\mathrm{~L})$ & $7.65(3)$ & 7.87 & 8.14 \\
\hline UGC 695E & 0.04 & $8.01(\mathrm{~L})$ & $7.93(3)$ & 8.13 & 8.34 \\
\hline UGC 1056 A & 0.04 & $8.08(\mathrm{~L})$ & $8.03(3)$ & 8.15 & 8.29 \\
\hline UGC 1176 A & 0.05 & $8.03(\mathrm{~L})$ & $7.90(3)$ & 8.04 & 8.18 \\
\hline NGC 784 A & 0.05 & 8.08 (L) & $7.95(3)$ & 8.02 & 8.15 \\
\hline UGC 2716 A & 0.04 & 8.08 (L) & $7.93(3)$ & 7.99 & 8.15 \\
\hline NGC 2537 A & 0.14 & $8.58(\mathrm{U})$ & $8.41(2)$ & 8.27 & 8.29 \\
\hline UGC $4278 \mathrm{~A}$ & 0.03 & $7.78(\mathrm{~L})$ & $7.64(3)$ & 7.88 & 8.19 \\
\hline NGC 2552 A & 0.10 & $7.98(\mathrm{~L})$ & $8.06(3)$ & 8.12 & 8.20 \\
\hline UGC 4393 B & 0.12 & $8.56(\mathrm{U})$ & $8.31(2)$ & 8.16 & 8.19 \\
\hline UGC 5139 A & 0.04 & 7.97 (L) & $7.78(3)$ & 7.94 & 8.16 \\
\hline IC 559 A & 0.05 & 8.09 (L) & $8.02(3)$ & 8.14 & 8.26 \\
\hline UGC 5272 A & 0.03 & $7.95(\mathrm{~L})$ & $7.71(3)$ & 7.73 & 8.04 \\
\hline UGC 5340 A & 0.03 & $7.34(\mathrm{~L})$ & $7.15(3)$ & 7.51 & 8.09 \\
\hline UGC 5423 A & 0.06 & 7.99 (L) & 7.97 (3) & 8.02 & 8.17 \\
\hline UGC 5797 A & 0.06 & $8.13(\mathrm{~L})$ & $8.02(3)$ & 7.92 & 8.07 \\
\hline UGC 5923 A & 0.06 & $8.20(\mathrm{~L})$ & $8.23(2)$ & 8.26 & 8.31 \\
\hline NGC 3738 A & 0.07 & 8.07 (L) & $8.05(3)$ & 8.15 & 8.24 \\
\hline NGC 3741 A & 0.03 & $7.81(\mathrm{~L})$ & $7.64(3)$ & 7.87 & 8.17 \\
\hline UGC 6817 A & 0.03 & $7.64(\mathrm{~L})$ & $7.45(3)$ & 7.69 & 8.10 \\
\hline NGC 4163 A & 0.03 & 8.07 (L) & $7.55(3)$ & 8.18 & 8.52 \\
\hline CGCG 269-049 A & 0.03 & $7.60(\mathrm{~L})$ & $7.65(3)$ & 7.72 & 8.05 \\
\hline UGC 7577 A & 0.05 & $8.12(\mathrm{~L})$ & $8.07(3)$ & 7.94 & 7.97 \\
\hline NGC 4449 A & 0.07 & $8.04(\mathrm{~L})$ & $8.19(2)$ & 8.08 & 8.19 \\
\hline UGC 7605 A & 0.03 & $7.81(\mathrm{~L})$ & $7.66(3)$ & 7.94 & 8.22 \\
\hline NGC 4656 A & 0.03 & $8.10(\mathrm{~L})$ & $7.81(3)$ & 7.62 & 7.96 \\
\hline UGC $8201 \mathrm{~A}$ & 0.02 & $7.82(\mathrm{~L})$ & $7.55(3)$ & 7.85 & 8.17 \\
\hline UGC 8508 A & 0.03 & $7.83(\mathrm{~L})$ & $7.62(3)$ & 7.85 & 8.14 \\
\hline UGC 8638 A & 0.04 & $8.01(\mathrm{~L})$ & $7.82(3)$ & 7.93 & 8.13 \\
\hline UGC 8837 A & 0.06 & $8.02(\mathrm{~L})$ & $7.92(3)$ & 8.20 & 8.38 \\
\hline NGC 5477 A & 0.04 & $7.97(\mathrm{~L})$ & $7.72(3)$ & 7.81 & 8.08 \\
\hline Offset & $\ldots$ & 0.14 & -0.001 & 0.12 & 0.32 \\
\hline Dispersion & $\ldots$ & 0.22 & 0.17 & 0.24 & 0.42 \\
\hline
\end{tabular}

Notes. Strong-line abundances are calculated for the 31 objects with spectra containing an [O III] $\lambda 4363$ signal to noise of 4 or greater. Four strong-line calibrations are given: (1) the $R_{23}$ method of McGaugh (1991) is listed in Column 3, where the appropriate branch was selected based on the $[\mathrm{N} \mathrm{II}] /[\mathrm{O}$ II $]$ ratio given in Column (2), with $[\mathrm{N}$ II $] /[\mathrm{O}$ II $] \approx 0.1$ drawing the division. Branch selection is denoted by (L) for lower branch and (U) for upper branch. (2) The ONS calibration of Pilyugin et al. (2010) is given in Column 4. Pilyugin et al. (2010) divide their calibration into three classes of H II regions, where we have used (1) for regions with $\log \left(N_{2}\right)>-0.1$, (2) for $\log \left(N_{2}\right)<-0.1$ and $\log \left(N_{2} / S_{2}\right)>-0.25$, and (3) for $\log \left(N_{2}\right)<-0.1$ and $\log \left(N_{2} / S_{2}\right)<-0.25$. (3) The N2 calibration, corrected for N/O ratio variations by PMC09, is given in Column 5. (4) The $\mathrm{O} 3 \mathrm{~N} 2$ calibration, also corrected for $\mathrm{N} / \mathrm{O}$ ratio variations by PMC09, is given in Column 6 . The last two rows give the average offset and dispersion of the strong-line abundances from their "Direct" abundance counterparts.

from the direct method. Thus, our data favor the ONS method, but do not support the claim of the very small error as found by Pilyugin et al. (2010). In Figure 7, we find that no clear trend exists between the "direct" method and the strong-line methods, implying that simple calibrations between methods are not possible.

With the relatively precise $M-Z$ and $L-Z$ relationships in place, and their correspondingly low dispersions, oxygen abundances for normal (non-starburst) low luminosity galaxies can be inferred with relatively high confidence without a spectrum. In fact, given reliable distance and photometry measurements, the resulting luminosity and mass estimates can be used as more

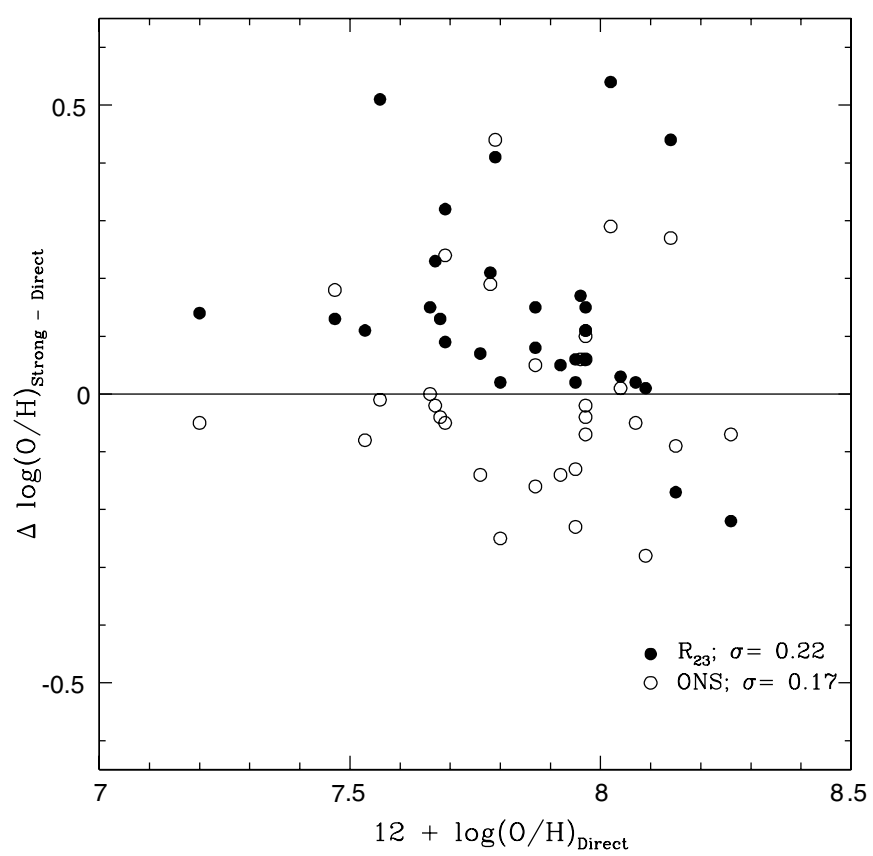

Figure 7. We plot the difference in oxygen abundances determined via the "direct" method and strong-line methods vs. the "direct" method oxygen abundances for the objects listed in Table 5. The open circles display the comparison for strong-line abundances determined via the $R_{23}$ method of McGaugh (1991) and the closed circles the ONS calibration of Pilyugin et al. (2010). The absence of clear trends implies that simple calibrations between methods are not possible.

reliable predictors of oxygen abundance than some strong-line calibrations. As counter-intuitive as this idea may seem, it is a natural consequence of the inability of some strong-line methods to accurately predict the metallicity of individual $\mathrm{H}$ II regions. Studies of abundances in dwarfs which do not reproduce the $L-Z$ and $M-Z$ relationships, therefore, should raise suspicions concerning methodology.

\section{CONCLUSIONS}

We have determined uniform oxygen abundance metallicities for 31 low-luminosity galaxies in the Spitzer LVL survey. With high-resolution spectral observations taken at the MMT, we were able to measure the intrinsically faint [O III] $\lambda 4363$ fluxes at strengths of $4 \sigma$ or greater and explicitly determine electron temperatures. Metallicity measurements are important for characterizing many other properties, especially when the more reliable "direct" method is used. However, metallicity relationships tend to suffer from small number statistics in the low-luminosity regime. In particular, these measurements allowed us to better characterize the luminosity-metallicity and mass-metallicity relationships by doubling the number of reliable low-luminosity measurements. We created a "Combined Select" sample of objects that have both reliable "direct" oxygen abundance determinations and distances estimated from the TRGB or Cepheid variables. With this sample, we find that both the luminosity-metallicity and the mass-metallicity relationships agree well with previous relationships defined for low luminosities.

From the 38 objects making up the "Combined Select" sample, we found an optical $L-Z$ relationship of $12+\log (\mathrm{O} / \mathrm{H})=$ $(6.27 \pm 0.21)+(-0.11 \pm 0.01) M_{B}$, with a dispersion of $\sigma=$ 0.15 . In comparison, the near-IR $L-Z$ relationship for these data is $12+\log (\mathrm{O} / \mathrm{H})=(6.10 \pm 0.21)+(-0.10 \pm 0.01) M_{[4.5]}$, 
Table 9

Strong-line Oxygen Abundances for [O III] $\lambda 4363$ Non-detection Objects

\begin{tabular}{lccccc}
\hline \hline & & \multicolumn{4}{c}{$12+\log (\mathrm{O} / \mathrm{H})$} \\
\cline { 3 - 6 } Galaxy & {$[\mathrm{N}$ II] $/[\mathrm{O}$ II $]$} & $R_{23}$ & ONS & $\mathrm{N} 2$ & O3N2 \\
\hline NGC 404 & 0.04 & $7.23(\mathrm{~L})$ & $7.55(3)$ & 8.53 & 8.56 \\
KKH 037 & 0.06 & $8.32(\mathrm{U})$ & $8.33(1)$ & 8.53 & 8.56 \\
CGCG 035-007 & 0.05 & $8.00(\mathrm{~L})$ & $8.10(3)$ & 8.57 & 8.37 \\
UGC 5672 & 0.06 & $8.00(\mathrm{~L})$ & $8.26(3)$ & 8.37 & 8.29 \\
UGC 5692 & 0.15 & $8.40(\mathrm{U})$ & $8.08(3)$ & 8.53 & 8.31 \\
UGC 6782 & 0.08 & $7.85(\mathrm{~L})$ & $\ldots$ & 8.18 & 8.27 \\
UGC 6900 & 0.12 & $8.00(\mathrm{U})$ & $7.69(3)$ & 8.08 & 8.17 \\
UGC 7599 & $\ldots$ & $8.09(\mathrm{U})$ & $\ldots$ & $\ldots$ & $\ldots$ \\
UGC 7639 & 0.05 & $7.77(\mathrm{~L})$ & $7.99(3)$ & 8.50 & 8.42 \\
UGC 8245 & 0.04 & $7.59(\mathrm{~L})$ & $7.78(3)$ & 8.40 & 8.39 \\
UGC 9405 & 0.11 & $7.77(\mathrm{~L})$ & $8.22(3)$ & 8.63 & 8.40 \\
UGC 10818 & 0.09 & $7.82(\mathrm{~L})$ & $\ldots$ & 8.45 & 8.29 \\
KKH 098 & 0.04 & $7.61(\mathrm{~L})$ & $7.66(3)$ & 8.17 & 8.27 \\
\hline
\end{tabular}

Notes. Strong-line abundances are calculated for $\mathrm{H}_{\text {II }}$ regions with no [O III] $\lambda 4363$ detections. Most objects in our sample have at least one $\mathrm{H}$ II region with a "direct" abundance determination, but 12 objects have good spectra and no [O III] $\lambda 4363$ measurement. Four strong-line calibrations are given for these objects: (1) the $R_{23}$ method of McGaugh (1991) is listed in Column 3, where the appropriate branch was selected based on the $[\mathrm{N} \mathrm{II}] /\left[\mathrm{O}_{\mathrm{II}}\right]$ ratio given in Column 2. $[\mathrm{N} \mathrm{II}] /[\mathrm{O} \mathrm{II}] \approx 0.1$ draws the branch division. Branch selection is denoted by (L) for lower branch and (U) for upper branch. (2) The ONS calibration of Pilyugin et al. (2010) is given in Column 4. Pilyugin et al. (2010) divide their calibration into three classes of $\mathrm{H}$ II regions, where we have used (1) for regions with $\log \left(N_{2}\right)>-0.1$, (2) for $\log \left(N_{2}\right)<-0.1$ and $\log \left(N_{2} / S_{2}\right)<-0.25$, and (3) for $\log \left(N_{2} / S_{2}\right)>-0.25$. (3) The $\mathrm{N} 2$ calibration, corrected for $\mathrm{N} / \mathrm{O}$ ratio variations by PMC09, is given in Column 5. (4) The O3N2 calibration, also corrected for $\mathrm{N} / \mathrm{O}$ ratio variations by PMC09, is given in Column 6.

with a dispersion of $\sigma=0.14$. While the slopes of the two $L-Z$ relationships agree, our findings confirm the work of L06 in that the near-IR relationship has lower scatter. By converting NIR luminosity to a stellar mass estimate, we determined the $M-Z$ relationship for our data to be $12+\log (\mathrm{O} / \mathrm{H})=$ $(5.61 \pm 0.24)+(0.29 \pm 0.03) M_{\star}$, with a dispersion of $\sigma=0.15$. In agreement with the idea that mass is more fundamentally related to metallicity than luminosity, we find that the intrinsic scatter of the optical $L-Z$, NIR $L-Z$, and $M-Z$ relationships decreases from 0.13 to 0.12 to 0.08 . However, the total dispersion of the $M-Z$ relationship was measured to be no smaller than the $L-Z$ relationships. This suggests, given a reliable distance measurement and appropriate photometry, that luminosity is just as strong a metallicity indicator as stellar mass. Furthermore, with the dispersions in luminosity and mass roughly equal, either may be used in combination with a reliable distance determination to estimate metallicity of a low-luminosity dwarf with more confidence than when using strong-line calibrations.

Our observations of N/O abundances are in agreement with previous studies. We find a positive correlation between $\mathrm{N} / \mathrm{O}$ ratio and $B-V$ color for $0.05 \lesssim B-V \lesssim 0.75 ; \log (\mathrm{N} / \mathrm{O})=$ $(-1.92 \pm 0.08)+(1.18 \pm 0.19) \times(B-V)$, with a dispersion of $\sigma=0.14$. Furthermore, in agreement with observations of blue compact galaxies, there are no objects with high $\mathrm{N} / \mathrm{O}$ ratio $(\log (\mathrm{N} / \mathrm{O})>-1.4)$ below $12+\log (\mathrm{O} / \mathrm{H})=7.7$. Since the typical low luminosity galaxy in the Local Volume displays roughly constant star formation over the age of the universe, the small dispersion in $\mathrm{N} / \mathrm{O}$ at low values of $\mathrm{O} / \mathrm{H}$ cannot be due to the very recent birth of the galaxy.

D.A.B. is grateful for support from a Penrose Fellowship and a NASA Space Grant Fellowship from the University

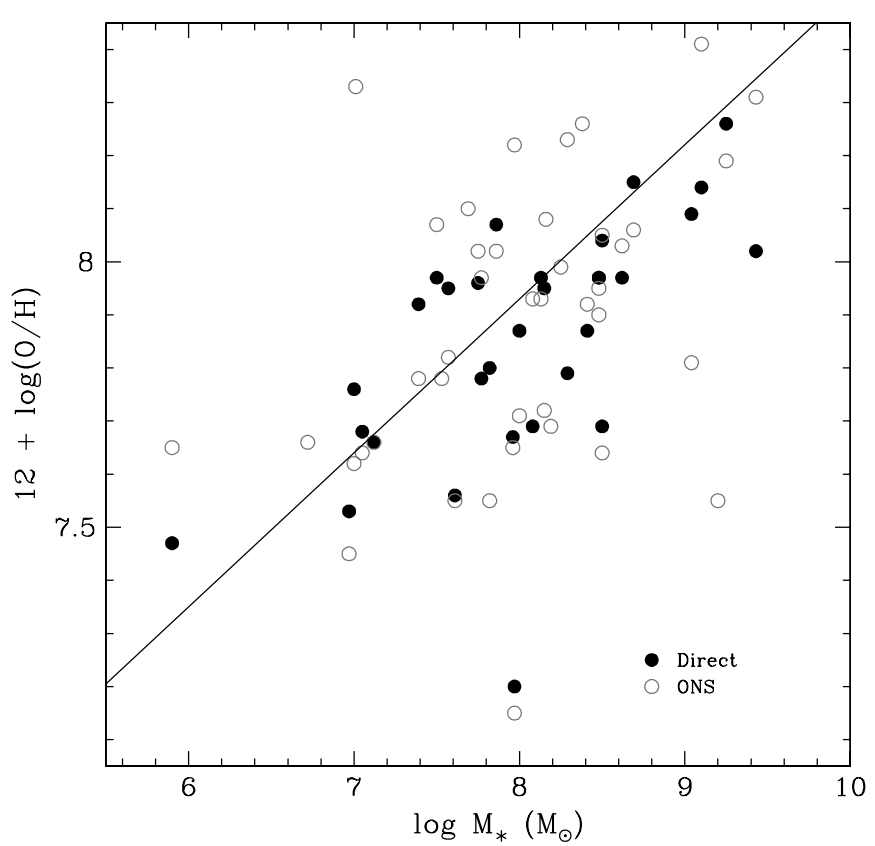

Figure 8. Strong-line ONS mass-metallicity relationship is depicted for all the objects for which we calculated strong-line abundances (see Appendix A). The objects are plotted in comparison to "direct" abundances determined for the "Combined Select" sample. As a reference, we have plotted Equation (14), our $M-Z$ relationship for the "Combined Select" sample, as a solid line. While scatter is apparent, the overall trend is well defined and the two samples coincide. Note that in terms of scatter the "direct" method is an improvement over the ONS strong-line calibration.

of Minnesota. E.D.S. is grateful for partial support from the University of Minnesota. We are grateful to the referee for a thorough analysis of this paper that greatly improved the analysis and presentation of this work. Special thanks to John Moustakas and L. Andrew Helton for many scientifically stimulating and helpful discussions. Observations reported here were obtained at the MMT Observatory, a joint facility of the Smithsonian Institution and the University of Arizona. MMT observations were obtained as part of the University of Minnesota's guaranteed time on Steward Observatory facilities through membership in the Research Corporation and its support for the Large Binocular Telescope, and granted by NOAO, through the Telescope System Instrumentation Program (TSIP). TSIP is funded by the National Science Foundation.

This research has made use of NASA's Astrophysics Data System Bibliographic Services and the NASA/IPAC Extragalactic Database (NED), which is operated by the Jet Propulsion Laboratory, California Institute of Technology, under contract with the National Aeronautics and Space Administration. This work was initiated as part of the Spitzer Space Telescope Legacy Science Program and was supported by National Aeronautics and Space Administration (NASA) through contract 1336000 issued by the Jet Propulsion Laboratory (JPL), California Institute of Technology (Caltech) under NASA contract 1407.

\section{APPENDIX A}

\section{STRONG-LINE ABUNDANCES FOR GALAXIES LACKING DIRECT ABUNDANCES}

In Table 9 we present strong-line abundances for the 12 objects in our sample without [O III] $\lambda 4363$ detections. While these may not be as accurate as the direct abundances for the 


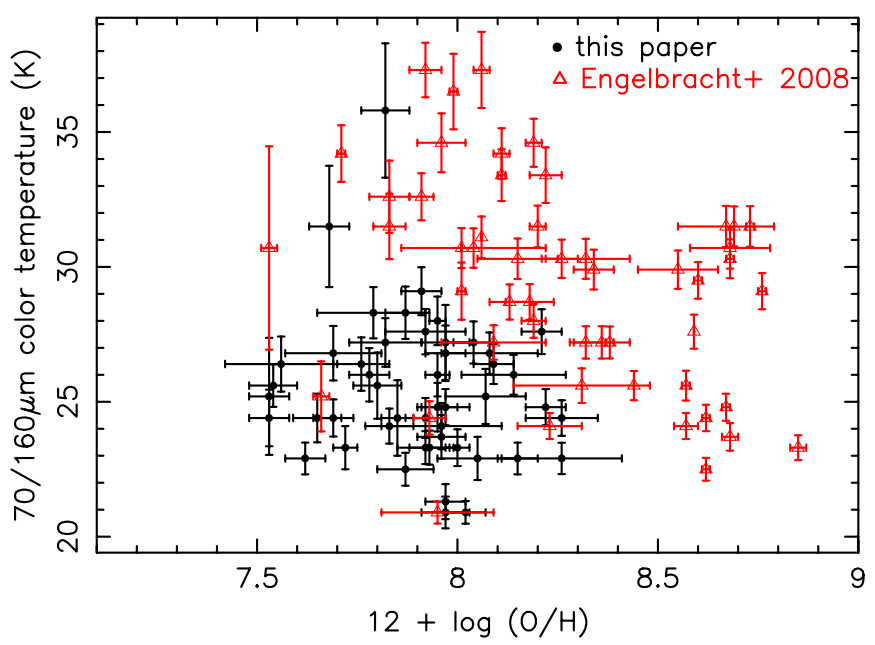

Figure 9. $70 / 160 \mu \mathrm{m}$ color temperature vs. $12+\log (\mathrm{O} / \mathrm{H})$ is plotted for the objects presented in this paper with "direct" oxygen abundances. In comparison, star-bursting galaxies from Engelbracht et al. (2008) with strongline abundances seem to have higher temperatures at a given metallicity.

(A color version of this figure is available in the online journal.)

rest of our sample, they may be useful for studies of these individual galaxies. The $\mathrm{O} / \mathrm{H}$ values derived using the ONS method for both these 12 objects (Table 9) and the objects with "direct abundances" (Table 8) are plotted in Figure 8 where they are compared to our "direct" abundances. The two methods display coincident trends in metallicity with mass, yet the $\mathrm{O} / \mathrm{H}$ abundances derived via the ONS calibration have a larger dispersion. We have not conducted a statistical comparison, as not all galaxies have accurate distances, and the subset with accurate distance is quite small.

\section{APPENDIX B \\ 70/160 $\mu \mathrm{m}$ COLOR-TEMPERATURE-METALLICITY OUTLIERS}

As noted in Section 2.2, two objects were of particular interest to this study (UGC 10818 and UGC 4393) because they appear to be outliers from the global trend of 70/160 $\mu \mathrm{m}$ color temperature as a function of metallicity as determined by Engelbracht et al. (2008). Specifically, based on Spitzer observations of 66 starburst galaxies, they showed that the farinfrared color temperature of large dust grains increases toward lower metallicity down to $12+\log (\mathrm{O} / \mathrm{H}) \sim 8$. However, the oxygen abundances found by Engelbracht et al. (2008) for these two objects were based on the $R_{23}$ strong-line estimator. Our new spectroscopic results indicate that both UGC 4393 and UGC 10818 (SHOC 567) are near the transition region between the upper and lower branches based on their [N II]/[O II ] ratios, and thus the $R_{23}$ method may not yield an accurate abundance for these systems.

While our observations of UGC 10818 are still ambiguous due to the degeneracy in the strong-line metallicity calibrations, we derive an oxygen abundance of $12+\log (\mathrm{O} / \mathrm{H})=7.82$ based on the McGaugh (1991) $R_{23}$ calibration. This increases the oxygen abundance of UGC 10818 by 0.51 dex compared to previous measurements and moves UGC 10818 (SHOC 567) closer to the original trend illustrated in Engelbracht et al. (2008). Conversely, the "direct" oxygen abundance of UGC 4393 was determined in this paper to be $12+$ $\log (\mathrm{O} / \mathrm{H})=8.02 \pm 0.05$, in agreement with the strong-line estimate presented in Engelbracht et al. (2008). Thus, at first glance, these new observations appear to only impact the location of one of the two most extreme outliers in the original plot.

Perhaps more importantly, we have reproduced the $70 / 160 \mu \mathrm{m}$ color temperature versus $12+\log (\mathrm{O} / \mathrm{H})$ plot of Engelbracht et al. (2008) with the addition of "direct" abundance objects from this work in Figure 9. Note that the star-bursting objects from Engelbracht et al. (2008) tend to have higher dust temperatures than the low intensity objects studied in this paper. This may mean that the trend of increasing far-infrared dust temperature with decreasing metallicity was just a slice of a larger picture, where the selected samples were limited by SFRs, which biased the view to a more narrow window. With a more complete range of intensities in star-forming galaxies now plotted, no clear trend emerges.

\section{REFERENCES}

Amorín, R. O., Pérez-Montero, E., \& Vílchez, J. M. 2010, ApJ, 715, L128 Annibali, F., Aloisi, A., Mack, J., et al. 2008, AJ, 135, 1900

Bell, E. F., \& de Jong, R. S. 2001, ApJ, 550, 212

Berg, D. A., Skillman, E. D., \& Marble, A. R. 2011, ApJ, 738, 2

Bresolin, F. 2007, ApJ, 656, 186

Bresolin, F., Gieren, W., Kudritzki, R.-P., et al. 2009, ApJ, 700, 309 Brinchmann, J., Charlot, S., White, S. D. M., et al. 2004, MNRAS, 351, 1151

Brinchmann, J., \& Ellis, R. S. 2000, ApJ, 536, 77

Brooks, A. M., Governato, F., Booth, C. M., et al. 2007, ApJ, 655, L17

Campbell, A., Terlevich, R., \& Melnick, J. 1986, MNRAS, 223, 811

Cannon, J. M., Dohm-Palmer, R. C., Skillman, E. D., et al. 2003, AJ, 126, 2806

Cardelli, J. A., Clayton, G. C., \& Mathis, J. S. 1989, ApJ, 345, 245

Cole, A. A., Skillman, E. D., Tolstoy, E., et al. 2007, ApJ, 659, L17

Cook, D. O., Seth, A. C., Dale, D. A., et al. 2012, ApJ, 751, 100

Cooper, M. C., Tremonti, C. A., Newman, J. A., \& Zabludoff, A. I. 2008, MNRAS, 390, 245

Conroy, C., \& Gunn, J. E. 2010, ApJ, 712, 833

Contini, T., Treyer, M. A., Sullican, M., \& Ellison, R. S. 2002, MNRAS, 330, 75

Crawford, D. L., \& Barnes, J. V. 1970, AJ, 75, 978

Croxall, K. V., van Zee, L., Lee, H., et al. 2009, ApJ, 705, 723

Dalcanton, J. J. 2007, ApJ, 658, 941

Dalcanton, J. J., Williams, B. F., Seth, A. C., et al. 2009, ApJS, 183, 67

Dale, D. A., Cohen, S. A., Johnson, L. C., et al. 2009, ApJ, 703, 517

Dekel, A., \& Silk, J. 1986, ApJ, 303, 39

de Vaucouleurs, G., de Vaucouleurs, A., Corwin, H. G., et al. 1991, Third Reference Catalogue of Bright Galaxies, Vols. 1-3, XII (Berlin: Springer), 2069

Dohm-Palmer, R. C., Skillman, E. D., Gallagher, J., et al. 1998, AJ, 116, 1227

Dolphin, A. E., Saha, A., Skillman, E. D., et al. 2003, AJ, 125, 1261

Draine, B. T., Dale, D. A., Bendo, G., et al. 2007, ApJ, 663, 866

Ekta, B., \& Chengalur, J. N. 2010, MNRAS, 406, 1238

Ekta, B., Chengalur, J. N., \& Pustilnik, S. A. 2008, MNRAS, 391, 881

Ellison, S. L., Patton, D. R., Simard, L., \& McConnachie, A. W. 2008, AJ, 135 , 1877

Engelbracht, C. W., Rieke, G. H., Gordon, K. D., et al. 2008, ApJ, 678, 804

Fazio, G. G., Hora, J. L., Allen, L. E., et al. 2004, ApJS, 154, 10

Filippenko, A. V. 1982, PASP, 94, 715

Fouesneau, M., \& Lançon, A. 2010, A\&A, 521, A22

Gallagher, J. S., III, \& Hunter, D. A. 1989, AJ, 98, 806

Garnett, D. R. 1990, ApJ, 363, 142

Garnett, D. R. 2002, ApJ, 581, 1019

Garnett, D. R., \& Shields, G. A. 1987, ApJ, 317, 82

Gil de Paz, A., Boissier, S., Madore, B. F., et al. 2007, ApJS, 173, 185

Gil de Paz, A., Zamorano, J., \& Gallego, J. 2000a, A\&A, 361, 465

Gil de Paz, A., Zamorano, J., Gallego, J., \& Domínguez, F. d. B. 2000b, A\&AS, 145,377

Henry, R. B. C., Edmunds, M. G., \& Köppen, J. 2000, ApJ, 541, 660

Henry, R. B. C., Nava, A., \& Prochaska, J. X. 2006, ApJ, 647, 984

Hopp, U., \& Schulte-Ladbeck, R. E. 1991, A\&A, 248, 1

Hummer, D. G., \& Storey, P. J. 1987, MNRAS, 224, 801

Hunter, D. A., \& Gallagher, J. S., III 1985, ApJS, 58, 533

Hunter, D. A., Gallagher, J. S., \& Rautenkranz, D. 1982, ApJS, 49, 53

Hunter, D. A., \& Hoffman, L. 1999, AJ, 117, 2789 
Izotov, Y. I., Stasińska, G., Meynet, G., Guseva, N. G., \& Thuan, T. X. 2006, A\&A, 448, 955

Izotov, Y. I., \& Thuan, T. X. 1999, ApJ, 511, 639

Izotov, Y. I., \& Thuan, T. X. 2007a, ApJ, 665, 1115

Izotov, Y. I., Thuan, T. X., \& Stasińska, G. 2007b, ApJ, 662, 15

James, B. L., Tsamis, Y. G., Barlow, M. J., et al. 2009, MNRAS, 398, 2

Jester, S., Schneider, D. P., Richards, G. T., et al. 2005, AJ, 130, 873

Karachentsev, I. D., Dolphin, A., Tully, R. B., et al. 2006, AJ, 131, 1361

Karachentsev, I. D., Kopylov, A. I., \& Kopylova, F. G. 1994, Bull. Special Astrophys. Obs., 38, 5

Karachentsev, I. D., Makarov, D. I., Sharina, M. E., et al. 2003, A\&A, 398, 479

Kehrig, C., Vílchez, J. M., Sánchez, S. F., et al. 2008, A\&A, 477, 813

Kennicutt, R. C., Jr., Lee, J. C., Funes, S. J., et al. 2008, ApJS, 178, 247

Kewley, L. J., \& Ellison, S. L. 2008, ApJ, 681, 1183

Kewley, L. J., Jansen, R. A., \& Geller, M. J. 2005, PASP, 117, 227

Kinman, T. D., \& Hintzen, P. 1981, PASP, 93, 405

Kniazev, A. Y., Grebel, E. K., Hao, L., et al. 2003, ApJ, 593, L73

Kniazev, A. Y., Grebel, E. K., Pustilnik, S. A., Pramskij, A. G., \& Zucker, D. B. 2005, AJ, 130, 1558

Kniazev, A. Y., Pustilnik, S. A., Grebel, E. K., Lee, H., \& Pramskij, A. G. 2004, ApJS, 153, 429

Kobulnicky, H. A., Kennicutt, R. C., Jr., \& Pizagno, J. L. 1999, ApJ, 514, 544

Kobulnicky, H. A., \& Skillman, E. D. 1996, ApJ, 471, 211

Kobulnicky, H. A., \& Skillman, E. D. 1997, ApJ, 489, 636

Kobulnicky, H. A., Skillman, E. D., Roy, J.-R., Walsh, J. R., \& Rosa, M. R. 1997, ApJ, 477, 679

Kobulnicky, H. A., Willmer, C. N. A., Phillips, A. C., et al. 2003, ApJ, 599, 1006

Köppen, J., Weidner, C., \& Kroupa, P. 2007, MNRAS, 375, 673

Lee, H., Grebel, E. K., \& Hodge, P. W. 2003a, A\&A, 401, 141

Lee, H., McCall, M. L., Kingsburgh, R. L., Ross, R., \& Stevenson, C. C. 2003b, AJ, 125, 146

Lee, H., \& Skillman, E. D. 2004, ApJ, 614, 698

Lee, H., Skillman, E. D., Cannon, J. M., et al. 2006a, ApJ, 647, 970

Lee, H., Skillman, E. D., \& Venn, K. A. 2005, ApJ, 620, 223

Lee, H., Skillman, E. D., \& Venn, K. A. 2006b, ApJ, 642, 813

Lee, J. C., Gil de Paz, A., Kennicutt, R. C., Jr., et al. 2011, ApJS, 192, 6

Lee, J. C., Salzer, J. J., \& Melbourne, J. 2004, ApJ, 616, 752

Leitherer, C., \& Ekstrom, S. 2011, arXiv:1111.5204

Leitner, S. N., \& Kravtsov, A. V. 2011, ApJ, 734, 48

Lequeux, J., Peimbert, M., Rayo, J. F., Serrano, A., \& Torres-Peimbert, S. 1979, A\&A, 80, 155

Liang, Y. C., Hu, J. Y., Liu, F. S., \& Liu, Z. T. 2007, AJ, 134, 759

Liang, Y. C., Yin, S. Y., Hammer, F., et al. 2006, ApJ, 652, 257

López-Sánchez, Á. R., Koribalski, B. S., van Eymeren, J., et al. 2012, MNRAS, 419, 1051

Makarova, L., Karachentsev, I., Takalo, L. O., Heinaemaeki, P., \& Valtonen, M. 1998, A\&AS, 128, 459

Makarova, L. N., \& Karachentsev, I. D. 1998, A\&AS, 133, 181

Marble, A. R., Engelbracht, C. W., van Zee, L., et al. 2010, ApJ, 715, 506

Markwardt, C. B. 2009, in ASP Conf. Ser. 411, Astronomical Data Analysis Software and Systems XVIII, ed. D. A. Bohlender, D. Durand, \& P. Dowler (San Francisco, CA: ASP), 251

Martin, C. L. 1997, ApJ, 491, 561

Matteucci, F., \& Tosi, M. 1985, MNRAS, 217, 391

McCall, M. L. 2004, AJ, 128, 2144

McCall, M. L., Rybski, P. M., \& Shields, G. A. 1985, ApJS, 57, 1

McGaugh, S. S. 1991, ApJ, 380, 140

McGaugh, S. S. 1994, ApJ, 426, 135

Meidt, S. E., Schinnerer, E., Knapen, J. H., et al. 2012, ApJ, 744, 17

Melbourne, J., Williams, B. F., Dalcanton, J. J., et al. 2012, ApJ, 748, 47

Miller, B. W., \& Hodge, P. 1996, ApJ, 458, 467

Mollá, M., Vílchez, J. M., Gavilán, M., \& Díaz, A. I. 2006, MNRAS, 372, 1069

Moustakas, J., \& Kennicutt, R. C., Jr. 2006, ApJS, 164, 81

Moustakas, J., Kennicutt, R. C., Jr., Tremonti, C. A., et al. 2010, ApJS, 190, 233

Moustakas, J., Zaritsky, D., Brown, M., et al. 2012, ApJ, accepted
Nava, A., Casebeer, D., Henry, R. B. C., \& Jevremovic, D. 2006, ApJ, 645, 1076 Noeske, K. G., Guseva, N. G., Fricke, K. J., et al. 2000, A\&A, 361, 33

Oey, M. S., \& Shields, J. C. 2000, ApJ, 539, 687

Oke, J. B. 1990, AJ, 99, 1621

Pagel, B. E. J., Simonson, E. A., Terlevich, R. J., \& Edmunds, M. G. 1992, MNRAS, 255, 325

Peimbert, M., \& Costero, R. 1969, Bol. Obs. Tonantzintla Tacubaya, 5,3

Pérez-Montero, E., \& Contini, T. 2009, MNRAS, 398, 949

Pérez-Montero, E., Vílchez, J. M., Cedrés, B., et al. 2011, A\&A, 532, A141

Pilyugin, L. S., \& Thuan, T. X. 2005, ApJ, 631, 231

Pilyugin, L. S., Vílchez, J. M., \& Thuan, T. X. 2010, ApJ, 720, 1738

Press, W. H., Teukolsky, S. A., Vetterling, W. T., \& Flannery, B. P. 1992,

Numerical Recipes in C: The Art of Scientific Computing (2nd ed.; Cambridge: Cambridge Univ. Press)

Pustilnik, S. A., Kniazev, A. Y., \& Pramskij, A. G. 2005, A\&A, 443, 91

Pustilnik, S. A., Tepliakova, A. L., \& Kniazev, A. Y. 2008a, Astron. Lett., 34, 457

Pustilnik, S. A., Tepliakova, A. L., Kniazev, A. Y., \& Burenkov, A. N. 2008b, MNRAS, 388, L24

Reach, W. T., Megeath, S. T., Cohen, M., et al. 2005, PASP, 117, 978

Rekola, R., Jerjen, H., \& Flynn, C. 2005, A\&A, 437, 823

Rizzi, L., Tully, R. B., Makarov, D., et al. 2007, ApJ, 661, 815

Rosenberg, J. L., Ashby, M. L. N., Salzer, J. J., \& Huang, J.-S. 2006, ApJ, 636, 742

Roy, J.-R., \& Kunth, D. 1995, A\&A, 294, 432

Russell, S. C., \& Dopita, M. A. 1990, ApJS, 74, 93

Sabbadin, F., Ortolani, S., \& Bianchini, A. 1984, A\&A, 131, 1

Sakai, S., Ferrarese, L., Kennicutt, R. C., Jr., \& Saha, A. 2004, ApJ, 608, 42

Sanna, N., Bono, G., Stetson, P. B., et al. 2008, ApJ, 688, L69

Saviane, I., Ivanov, V. D., Held, E. V., et al. 2008, A\&A, 487, 901

Schmidt, G. D., Weymann, R. J., \& Foltz, C. B. 1989, PASP, 101, 713

Sharina, M. E., Karachentsev, I. D., \& Tikhonov, N. A. 1996, A\&AS, 119, 499

Sharina, M. E., Karachentsev, I. D., \& Tikhonov, N. A. 1999, Astron. Lett., 25, 322

Skillman, E. D. 1985, ApJ, 290, 449

Skillman, E. D., Côté, S., \& Miller, B. W. 2003, AJ, 125, 610

Skillman, E. D., Kennicutt, R. C., \& Hodge, P. W. 1989, ApJ, 347, 875

Skillman, E. D., Terlevich, R., Teuben, P. J., \& van Woerden, H. 1988, A\&A, 198,33

Springob, C. M., Masters, K. L., Haynes, M. P., Giovanelli, R., \& Marinoni, C. 2009, ApJS, 182, 474

Stasińska, G. 1990, A\&AS, 83, 501

Stasińska, G. 2010, in IAU Symp. 262, Stellar Populations-Planning for the Next Decade, ed. G. Bruzual \& S. Charlot (Cambridge: Cambridge Univ. Press), 93

Thuan, T. X., \& Izotov, Y. I. 2005, ApJS, 161, 240

Tolstoy, E., Gallagher, J. S., Cole, A. A., et al. 1998, AJ, 116, 1244

Tosi, M., Sabbi, E., Bellazzini, M., et al. 2001, AJ, 122, 1271

Tremonti, C. A., Heckman, T. M., Kauffmann, G., et al. 2004, ApJ, 613, 898

Tüllmann, R., Rosa, M. R., Elwert, T., et al. 2003, A\&A, 412, 69

Tully, R. B., Rizzi, L., Dolphin, A. E., et al. 2006, AJ, 132, 729

Vaduvescu, O., McCall, M. L., \& Richer, M. G. 2007, AJ, 134, 604

van Zee, L. 2000, ApJ, 543, L31

van Zee, L., \& Haynes, M. P. 2006, ApJ, 636, 214

van Zee, L., Haynes, M. P., \& Salzer, J. J. 1997a, AJ, 114, 2497

van Zee, L., Haynes, M. P., \& Salzer, J. J. 1997b, AJ, 114, 2479

van Zee, L., Salzer, J. J., Haynes, M. P., O’Donoghue, A. A., \& Balonek, T. J. 1998, AJ, 116, 2805

van Zee, L., Skillman, E. D., \& Haynes, M. P. 2006, ApJ, 637, 269

Vázquez, G. A., \& Leitherer, C. 2005, ApJ, 621, 695

Vila Costas, M. B., \& Edmunds, M. G. 1993, MNRAS, 265, 199

Weisz, D. R., Dalcanton, J. J., Williams, B. F., et al. 2011, ApJ, 739, 5

Williams, M. J., Bureau, M., \& Cappellari, M. 2010, MNRAS, 409, 1330

Yin, S. Y., Liang, Y. C., Hammer, F., et al. 2007, A\&A, 462, 535

York, D. G., Adelman, J., Anderson, J. E., Jr., et al. 2000, AJ, 120, 1579

Zaritsky, D., Kennicutt, R. C., Jr., \& Huchra, J. P. 1994, ApJ, 420, 87 